\title{
DUALITÉ ET PRINCIPE LOCAL-GLOBAL POUR LES TORES SUR UNE COURBE AU-DESSUS DE $\mathbb{C}((t))$
}

\author{
par \\ Jean-Louis Colliot-Thélène \& David Harari
}

Résumé. - Pour $K$ un corps global (corps de nombres ou corps de fonctions d'une variable sur un corps fini $F$ ), on dispose de théorèmes de dualité classiques (Tate, Poitou, Nakayama) pour la cohomologie galoisienne à valeurs dans des tores et des modules abéliens finis.

Nous établissons de tels théorèmes pour $K$ le corps des fonctions d'une courbe projective et lisse sur le corps $F=\mathbb{C}((t))$ des séries formelles en une variable sur le corps des complexes. Cela permet de contrôler le défaut du principe de Hasse et l'approximation faible pour les espaces homogènes sous un tore.

Il y a ici des différences avec le cas classique ( $K$ corps global), et aussi avec le cas récemment étudié où $K$ est un corps de fonctions d'une variable sur un corps $p$-adique. Par exemple, la $K$-rationalité d'un tore n'implique pas ici la validité du principe de Hasse pour ses espaces principaux homogènes.

\footnotetext{
Abstract. - Over a global field $K$ (number field, or function field of a curve over a finite field $F$ ), arithmetic duality theorems for the Galois cohomology of tori and finite Galois modules have long been known. More recent work investigates the case where $K$ is the function fields of of a curve over a $p$-adic field.

For $K$ the function field of a curve over the formal series field $F=\mathbb{C}((t))$, we establish analogous duality theorems. We thus control the obstruction to the local-global principle and to weak approximation for homogeneous spaces of tori.

There are differences with the afore described cases. For example the Hasse principle need not hold for principal homogeneous spaces of a $K$-rational torus.
} 


\section{Introduction}

Soient $k$ le corps des fractions d'un anneau de valuation discrète, de corps résiduel $\kappa$, et $K$ le corps des fonctions d'une courbe $C$ projective, lisse, géométriquement intègre sur $k$. Dans plusieurs travaux récents, on s'est intéressé au principle local-global pour les points rationnels des espaces principaux homogènes (torseurs) sous un $k$-groupe linéaire $G$. Citons ici les travaux de Harbater, Hartmann et Krashen [18, 19, 20], ceux de Parimala, Suresh et l'un des auteurs du présent article [6], et ceux de l'autre auteur avec Szamuely [17] et avec Scheiderer et Szamuely [14].

On peut s'intéresser au principe local-global pour au moins deux familles de complétions $K_{v}$ de $K$ par rapport à des valuations discrètes de rang 1 .

(i) Les complétions par rapport aux valuations triviales sur $k$, c'est-à-dire les valuations correspondant aux points fermés de la $k$-courbe $C$.

(ii) Les complétions par rapport à toutes les valuations sur $K, \mathrm{y}$ compris celles qui induisent la valuation du corps complet $k$.

Harbater, Hartmann et Krashen ont montré que si le $K$-groupe $G$ est connexe et $K$-rationnel, alors dans le cas (ii) on a un principe local-global pour les torseurs sous $G$.

Supposons désormais $\kappa=\mathbb{C}$ et $k=\mathbb{C}((t))$ (plus généralement tous les résultats de cet article sont valables en remplaçant $\mathbb{C}$ par un corps algébriquement clos de caractéristique zéro).

Dans ce cas $K$ est un corps de dimension cohomologique 2 et présente des analogies avec les corps globaux de caractéristique positive, i.e. avec les corps de fonctions d'une variable sur un corps fini. Une différence importante est l'absence d'un théorème de Tchebotarev sur un tel corps. Ainsi un élément de $K$ peut par exemple être un carré dans tous les complétés $K_{v}$ pour $v \in \Omega$ sans être un carré dans $K$.

Dans le cas (i), le principe local-global ne vaut pas en général pour les points rationnels des torseurs sous un $K$-tore $K$-rationnel (voir l'exemple 2.10) à la différence de ce qui se passe sur un corps de nombres (Voskresenskǐ̌, voir [28], Cor. 9.7) ou sur le corps des fonctions d'une courbe $p$-adique ([17], Cor. 5.7). Autrement dit, avec les notations ci-dessous, on peut avoir $\amalg^{1}(K, T) \neq 0$ même pour un tore $T$ qui est $K$-rationnel. Dans [6], on donne aussi des exemples de $K$-tore algébrique - non $K$-rationnel pour lequel le principe local-global pour les points rationnels des torseurs ne vaut pas dans le cas (ii).

$\mathrm{Au}$ vu des résultats classiques sur un corps global comme ceux de Vos-

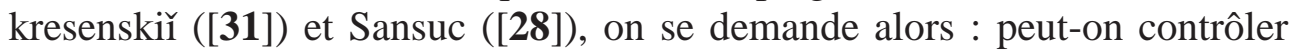


le défaut du principe de Hasse (et aussi de l'approximation faible) au moyen d'un accouplement cohomologique?

Soit $\Omega$ l'ensemble des points fermés de la $k$-courbe $C$. Pour tout $K$-schéma en groupes commutatif $G$, et tout entier $i \geq 0$, on définit le groupe abélien

$$
\amalg^{i}(K, G)=\operatorname{Ker}\left[H^{i}(K, G) \rightarrow \prod_{v \in \Omega} H^{i}\left(K_{v}, G\right)\right] .
$$

Si $K$ est sous-entendu, on abrègera parfois $\amalg^{i}(K, G)$ en $\amalg^{i}(G)$.

Soit $\mu_{n}$ le groupe des racines $n$-ième de l'unité dans $k$, on note $\mathbb{Q} / \mathbb{Z}(-1)$ la limite inductive des $\mathbb{Z} / n(-1):=\operatorname{Hom}\left(\mu_{n}, \mathbb{Z} / n\right)$. C'est un groupe abélien isomorphe (non canoniquement) à $\mathbb{Q} / \mathbb{Z}$.

Les principaux résultats de dualité obtenus dans cet article sont rassemblés dans le théorème suivant, établi au $\S 7$.

Théorème. Soient $T$ un $K$-tore et $\widehat{T}$ son groupe des caractères. Il existe des accouplements de groupes de torsion

$$
\amalg^{1}(K, T) \times \amalg^{2}(K, \widehat{T}) \rightarrow \mathbb{Q} / \mathbb{Z}(-1)
$$

et

$$
\amalg^{1}(K, \widehat{T}) \times \amalg^{2}(K, T) \rightarrow \mathbb{Q} / \mathbb{Z}(-1)
$$

qui par passage au quotient par les sous-groupes divisibles maximaux induisent des accouplements parfaits de groupes finis

$$
\amalg^{1}(K, T) \times \amalg^{2}(K, \widehat{T}) / \operatorname{Div} \rightarrow \mathbb{Q} / \mathbb{Z}(-1)
$$

et

$$
\amalg^{1}(K, \widehat{T}) \times \amalg^{2}(K, T) / \operatorname{Div} \rightarrow \mathbb{Q} / \mathbb{Z}(-1) .
$$

Il s'agit en principe d'adapter les arguments donnés dans [17]. Dans [17], on s'intéresse au cas où $K$ est le corps des fonctions d'une courbe sur un corps $p$-adique. Dans ce cas-là, il y a un seul type de dualité à étudier, à savoir

$$
\amalg^{1}(K, T) \times \amalg^{2}\left(K, T^{\prime}\right) \rightarrow \mathbb{Q} / \mathbb{Z},
$$

où $T^{\prime}$ est le $K$-tore dual de $T$. En effet les rôles de $T$ et $T^{\prime}$ sont interchangeables. Ici il nous faut étudier deux accouplements distincts (comme dans le cas des corps globaux).

Une conséquence (Cor. 8.3) du théorème précédent est que comme dans le cas des corps de nombres, l'obstruction au principe de Hasse pour un espace principal homogène $Y$ d'un $K$-tore est contrôlée par un certain sous-groupe 
du groupe de Brauer algébrique $\operatorname{Br}_{1} Y$, constitué des éléments dont la restriction à $\operatorname{Br}\left(Y \times_{K} K_{v}\right)$ est constante en toute place $v \in C^{(1)}$.

On verra aussi (Proposition 9.14) qu'à la différence du cas des corps globaux, il existe des contre-exemples à l'approximation faible pour un tore qui ne sont pas contrôlés par le groupe de Brauer non ramifié du tore. Comme la dimension cohomologique de $K$ est 2 , on ne peut ici espérer utiliser comme substitut du groupe de Brauer le groupe $H^{3}$ non ramifié, comme le font Scheiderer, Szamuely et le second auteur [14] sur les corps de fonctions d'une variable sur un corps $p$-adique.

Néanmoins on verra qu'on a encore une suite exacte à la Voskresenskǐ̌Sansuc décrivant le défaut d'approximation faible (Théorème 9.11).

Il resterait à comprendre s'il y a une relation entre les obstructions au principe local-global par rapport aux places de $K$ triviales sur $k$, comme étudiées dans le présent article, et les obstructions supérieures, par rapport à toutes les valuations de $K$, et faisant intervenir des lois de réciprocité aux points de codimension 2 sur les surfaces arithmétiques, utilisées dans [6].

\section{Notations}

Soit $A$ un groupe abélien (qu'on supposera toujours équipé de la topologie discrète si on ne définit pas une autre topologie dessus). Pour $n>0$ entier on note ${ }_{n} A \subset A$ le sous-groupe des éléments annulés par $n$. Pour $\ell$ premier, on note $A\{l\} \subset A$ la torsion $\ell$-primaire de $A$. On note $A^{(\ell)}$ la limite projective des $A / \ell^{n} A$. On note $A^{D}=\operatorname{Hom}_{c}(A, \mathbb{Q} / Z(-1))$ le groupe des homomorphismes continus de $A$ dans $\mathbb{Q} / \mathbb{Z}(-1)$. Le foncteur $(.)^{D}$ est exact sur la catégorie des groupes discrets, et il réalise de plus une anti-équivalence de catégories entre les groupes discrets de torsion et les groupes profinis.

Dans tout ce texte, la cohomologie utilisée est la cohomologie étale (ou galoisienne si on travaille sur un corps). Soit $C$ une courbe projective et lisse sur un corps $k$ (dont on notera $\bar{k}$ une clôture algébrique) ; soient $U$ un ouvert de Zariski non vide de $C$ et $j_{U}: U \rightarrow C$ l'inclusion. Pour tout faisceau étale $\mathcal{F}$ sur $U$, on note $\left(j_{U}\right)_{!} F$ le prolongement de $\mathcal{F}$ par zéro et pour tout $i \geq 0$ entier on note

$$
H_{c}^{i}(U, \mathcal{F}):=H^{i}\left(C,\left(j_{U}\right) ! \mathcal{F}\right)
$$

les groupes de cohomologie étale à support compact (cf. [23], Prop. III.1.29). 
Remerciements. Les auteurs remercient le Centre Interfacultaire Bernoulli de l'École Polytechnique Fédérale de Lausanne pour son hospitalité à l'automne 2012, ainsi que R. Parimala et T. Szamuely pour d'intéressantes discussions. Pour ce travail, le premier auteur a bénéficié d'une aide de l'Agence Nationale de la Recherche portant la référence ANR-12-BL01-0005.

\section{Les corps}

Soient $k=\mathbb{C}((t))$ et $K=\mathbb{C}((t))(C)$ le corps des fonctions d'une courbe $C$ projective, lisse, géométriquement connexe sur $k$. Dans la terminologie de [25], ce sont des corps $(\mathrm{lg})$.

Pour tout point fermé $v$ de $C$, le corps résiduel est une extension finie de $\mathbb{C}((t))$, donc de la forme $\mathbb{C}((s))$ avec $s=t^{1 / n}$ pour $n$ convenable, et le corps des fractions du complété de l'anneau local de $C$ en $v$ est isomorphe à $\mathbb{C}((s))((u))$.

Une extension finie d'un corps $K=\mathbb{C}((t))(C)$ est de la forme $\mathbb{C}\left(\left(t^{\prime}\right)\right)\left(C^{\prime}\right)$ pour $C^{\prime}$ une courbe géométriquement connexe convenable sur un corps $\mathbb{C}\left(\left(t^{\prime}\right)\right)$.

Une extension finie d'un corps $\mathbb{C}((s))((u))$ est de la forme $\mathbb{C}\left(\left(s^{\prime}\right)\right)\left(\left(u^{\prime}\right)\right)$.

Un corps $K$ de la forme $\mathbb{C}((t))(C)$ ou $\mathbb{C}((s))((u))$ a les propriétés suivantes. Toute extension finie de $K$ est encore du même type. Le corps $K$ est un corps $C_{2}$ - puisque $\mathbb{C}((t))$ est un corps $C_{1}$. Le corps $K$ est de dimension cohomologique 2. L'extension abélienne maximale de $K$ est un corps $C_{1}$, donc de dimension cohomologique 1 . Ceci résulte simplement du fait que la clôture algébrique de $\mathbb{C}((t))$ est, d'après le théorème de Puiseux, une extension abélienne de $\mathbb{C}((t))$.

Pour toute algèbre simple centrale sur $K$, la norme réduite est surjective : ceci résulte du fait que $K$ est un corps $C_{2}$.

Pour toute algèbre simple centrale $D$ sur $K$, indice et exposant de l'algèbre D coüncident. Pour la partie 2-primaire et la partie 3-primaire, c'est une conséquence connue (M. Artin) de la propriété $C_{2}$. Pour le cas local, il suffit de renvoyer à [4, Thm. 1.5]. Pour le cas $\mathbb{C}((t))(C)$, comme le mentionne Parimala [25], cela peut se voir soit en utilisant la méthode de scindage de la ramification de Saltman [27], soit par la méthode de recollement de corps de Harbater, Hartmann et Krashen [18, Thm. 5.5].

Soit $L / K$ une extension finie galoisienne de $K=\mathbb{C}((t))(C)$. Ceci correspond à un revêtement fini éventuellement ramifié de courbes intègres régulières $D \rightarrow C$ propres au-dessus du corps $k=\mathbb{C}((t))$. Pour $v \in C^{(1)}$ 
un point fermé où $D \rightarrow C$ est étale, et $w \in D^{(1)}$ point fermé au-dessus de $v$, le groupe de décomposition s'identifie au groupe de Galois des extensions de corps résiduelles. Comme toute extension finie de $\mathbb{C}((t))$, cette extension est cyclique. Ainsi presque tous les groupes de décomposition sont cycliques, comme dans le cas des extensions galoisiennes de corps globaux.

Par contre on n'a pas l'analogue du théorème de Tchebotarev: Pour une extension $L / K$ cyclique, il se peut qu'aucun groupe de décomposition ne soit égal au groupe de Galois de $L / K$. Voici un exemple : on prend une isogénie cyclique $D_{0} \rightarrow C_{0}$ de courbes elliptiques sur $\mathbb{C}$. On étend la situation à $\mathbb{C}((t))$. Pour toute extension finie $\mathbb{C}((s)) / \mathbb{C}((t)$, l'application $D(\mathbb{C}((s))) \rightarrow$ $C(\mathbb{C}((s)))$ est surjective. Ainsi tous les groupes de décomposition sont triviaux.

Des exemples similaires se trouvent dans [26].

\section{Cohomologie à coefficients $\mu_{n}$ et $\mathbb{G}_{m}$}

On dit qu'un corps $k$ est à cohomologie galoisienne finie si pour tout module galoisien fini $M$ et tout $i \geq 0$, le groupe $H^{i}(k, M)$ est fini.

2.1. Cohomologie étale des courbes. - Soient $k=\mathbb{C}((t))$ et $\bar{k}=$ $\cup_{n} \mathbb{C}\left(\left(t^{1 / n}\right)\right)$. Soit $\mathfrak{g}=\operatorname{Gal}(\bar{k} / k)$. On a un isomorphisme naturel $\mathfrak{g}=\widehat{\mathbb{Z}}(1)$.

Proposition 2.1. - Soit $C / k$ une courbe projective lisse géométriquement connexe de jacobienne $J$, et soit $K=k(C)$ son corps des fonctions. Soit $\bar{C}=C \times{ }_{k} \bar{k}$. On note $I(C)$ l'indice de $C$ (i.e. le pgcd des degrés sur $k$ des points fermés de $C$ ) de la courbe.

(i) Pour tout entier $n \geq 1$, et tout entier $i \in \mathbb{Z}$, on a des isomorphismes canoniques $H^{2}\left(\bar{C}, \mu_{n}^{\otimes i}\right)=\mu_{n}^{\otimes i-1}$ et $H^{2}(\bar{C}, \mathbb{Q} / \mathbb{Z}(i))=\mathbb{Q} / \mathbb{Z}(i-1)$.

(ii) Pour tout entier $n \geq 1$, et tout entier $i \in \mathbb{Z}$, on a des isomorphismes canoniques $H^{3}\left(C, \mu_{n}^{\otimes i}\right)=\mu_{n}^{\otimes i-2}$ et $H^{3}(C, \mathbb{Q} / \mathbb{Z}(i))=\mathbb{Q} / \mathbb{Z}(i-2)$.

(iii) Soit $U \subset C, U \neq C$ un ouvert de $C$. On a $H^{3}\left(U, \mathbb{G}_{m}\right)=0$ et $H_{c}^{3}\left(U, \mathbb{G}_{m}\right)=H^{3}\left(C, \mathbb{G}_{m}\right)=\mathbb{Q} / \mathbb{Z}(-1)$. Pour tout entier $n \geq 1$ et tout $i \in \mathbb{Z}$ on a $H_{c}^{3}\left(U, \mu_{n}^{\otimes i}\right)=H^{3}\left(C, \mu_{n}^{\otimes i}\right)=\mu_{n}^{\otimes i-2}$, et $H^{3}\left(U, \mu_{n}^{\otimes i}\right)=0$. De plus les groupes $H^{r}\left(U, \mu_{n}^{\otimes i}\right)$ sont finis pour tout $r \geq 0$ et tout $i \in \mathbb{Z}$, et le groupe de Brauer $\mathrm{BrC}$ est un groupe divisible de type cofini, quotient de $H^{1}(k, J)$ par un groupe cyclique d'ordre $I(C)$.

(iv) Pour chaque place $v \in \Omega$, on a $\operatorname{Br}\left(K_{v}\right) \stackrel{\simeq}{\rightarrow} H^{1}\left(\kappa_{v}, \mathbb{Q} / \mathbb{Z}\right)=\mathbb{Q} / \mathbb{Z}(-1)$. 
(v) On a une suite exacte naturelle

$$
0 \rightarrow \operatorname{Br} C \rightarrow \operatorname{Br} K \rightarrow \bigoplus_{v \in C^{1}} \operatorname{Br} K_{v} \rightarrow \mathbb{Q} / \mathbb{Z}(-1) \rightarrow 0
$$

Démonstration. - Pour tout entier $n \geq 1$ on a la suite exacte de Kummer

$$
1 \rightarrow \mu_{n} \rightarrow \mathbb{G}_{m} \stackrel{x \mapsto x^{n}}{\longrightarrow} \mathbb{G}_{m} \rightarrow 1 .
$$

Le théorème de Tsen et les propriétés connues du groupe de Brauer donnent $H^{2}\left(\bar{C}, \mathbb{G}_{m}\right)=0$. De la suite de Kummer et de la structure du schéma de Picard on déduit

Ceci donne (i).

$$
\mathbb{Z} / n=\operatorname{Pic} \bar{C} / n \stackrel{\sim}{\rightarrow} H^{2}\left(\bar{C}, \mu_{n}\right) .
$$

Comme on a $\operatorname{cd}(k) \leq 1$ et $H^{i}\left(\bar{C}, \mu_{n}^{\otimes i}\right)=0$ pour $i \geq 3$, la suite spectrale

$$
E_{2}^{p q}=H^{p}\left(k, H^{q}\left(\bar{C}, \mu_{n}^{\otimes i}\right)\right) \Longrightarrow H^{n}\left(C, \mu_{n}^{\otimes i}\right)
$$

donne

$$
H^{3}\left(C, \mu_{n}\right)=H^{1}\left(k, H^{2}\left(\bar{C}, \mu_{n}^{\otimes i}\right)\right)=H^{1}\left(k, \mu_{n}^{\otimes i-1}\right)=\mu_{n}^{\otimes i-2},
$$

ce qui donne (ii).

On a $H^{q}\left(\bar{C}, \mathbb{G}_{m}\right)=0$ pour $q \geq 2$. La suite spectrale

$$
E_{2}^{p q}=H^{p}\left(k, H^{q}\left(\bar{C}, \mathbb{G}_{m}\right)\right) \Longrightarrow H^{n}\left(C, \mathbb{G}_{m}\right)
$$

et la nullité de $H^{2}\left(k, \operatorname{Pic}^{0} \bar{C}\right)$ (qui vient de ce que $\operatorname{Pic}^{0} \bar{C}$ est divisible) donne

$$
H^{3}\left(C, \mathbb{G}_{m}\right)=H^{2}(k, \operatorname{Pic} \bar{C})=H^{2}(k, \mathbb{Z})=H^{1}(k, \mathbb{Q} / \mathbb{Z})=\mathbb{Q} / \mathbb{Z}(-1) .
$$

La suite spectrale analogue pour $U$ donne la finitude de $H^{r}\left(U, \mu_{n}^{\otimes i}\right)$ via [23], Th. VI.5.5 qui vaut sur un corps algébriquement clos. Cette même suite spectrale donne $H^{3}\left(U, \mathbb{G}_{m}\right)=H^{2}(k, \operatorname{Pic} \bar{U})$. Le groupe Pic $\bar{C}$ est extension de $\mathbb{Z}$ par un groupe divisible. Pour $U$ ouvert strict de $C$, le groupe Pic $\bar{U}$ est donc extension d'un groupe fini par un groupe divisible. Ceci implique $H^{2}(k, \operatorname{Pic} \bar{U})=0$, et donc $H^{3}\left(U, \mathbb{G}_{m}\right)=0$. De même $H^{3}\left(U, \mu_{n}^{\otimes i}\right)=0$ car $\operatorname{cd}(k) \leq 1$ et $H^{q}\left(\bar{U}, \mu_{n}^{\otimes i}\right)=0$ pour $q>1$ vu que $\bar{U}$ est une courbe affine sur un corps algébriquement clos.

Les corps $\kappa(v)$ sont de dimension cohomologique 1 , les $H^{q}\left(\kappa(v), \mathbb{G}_{m}\right)$ sont donc nuls pour $q \geq 2$. La proposition 3.1 (2) de [17] donne l'isomorphisme $H_{c}^{3}\left(U, \mathbb{G}_{m}\right) \stackrel{\sim}{\rightarrow} H^{3}\left(C, \mathbb{G}_{m}\right)$ et $H_{c}^{3}\left(U, \mu_{n}^{\otimes i}\right)=H^{3}\left(C, \mu_{n}^{\otimes i}\right)$ pour tout $i \in \mathbb{Z}$. Enfin on déduit aussi de la suite spectrale et de la nullité de $\operatorname{Br} \bar{C}$ (Tsen) un isomorphisme

$$
H^{1}(k, \operatorname{Pic} \bar{C})=\operatorname{Br} C .
$$


De la suite exacte

$$
0 \rightarrow J(\bar{k}) \rightarrow \operatorname{Pic} \bar{C} \rightarrow \mathbb{Z} \rightarrow 0
$$

et de $\operatorname{cd}(k) \leq 1$ on déduit la suite exacte

$$
0 \rightarrow \mathbb{Z} / I(C) \rightarrow H^{1}(k, J) \rightarrow H^{1}(k, \operatorname{Pic} \bar{C}) \rightarrow 0
$$

car $H^{1}(k, \mathbb{Z})=0$. De $\operatorname{cd}(k) \leq 1$ et de la divisibilité de $J$ on déduit que $H^{1}(k, J)$ est divisible, ce qui implique la divisiblité de $\mathrm{BrC}$. Ceci établit (iii).

L'énoncé (iv) est standard. Le calcul de $H^{3}\left(C, \mathbb{G}_{m}\right)$ et la nullité de $H^{3}\left(K, \mathbb{G}_{m}\right)$, ainsi que les isomorphismes $H^{2}\left(K_{v}, \mathbb{G}_{m}\right) \stackrel{\simeq}{\rightarrow} H^{1}\left(\kappa_{v}, \mathbb{Q} / \mathbb{Z}\right)$ et [13. III, Prop. (2.1)] donnent l'énoncé (v). On peut aussi l'obtenir à moindres frais en prenant la cohomologie galoisienne de la suite exacte

$$
1 \rightarrow \bar{k}(C)^{\times} / \bar{k}^{\times} \rightarrow \operatorname{Div} \bar{C} \rightarrow \operatorname{Pic} \bar{C} \rightarrow 0 .
$$

Remarque 2.2. - Certains des résultats de cette sous-section sont discutés dans [24, Chap. I, Appendix A, p. 131-138].

\subsection{Les groupes $\amalg^{1}\left(K, \mu_{n}^{\otimes i}\right)$. -}

Proposition 2.3. - Soient F un corps de caractéristique nulle, $k=F((t))$, et $C$ une $k$-courbe projective, lisse, géométriquement intègre. Soit $K$ le corps des fonctions de $C$. Soit $\mathcal{C} / F[[t]]$ un $F[[t]]$-modèle régulier, intègre, projectif de $C / k$. Soit $\mathcal{C}_{0} / F$ la fibre spéciale et $C_{0}=\left(\mathcal{C}_{0}\right)_{\text {red }}$ la fibre spéciale réduite de $\mathcal{C} / F[[t]]$.

Considérons les groupes suivants.

(i) Le groupe $\amalg^{1}\left(K, \mu_{n}\right)=\operatorname{Ker}\left[H^{1}\left(K, \mu_{n}\right) \rightarrow \prod_{v \in \Omega} H^{1}\left(K_{v}, \mu_{n}\right)\right]$.

(ii) Le noyau de la flèche d'évaluation $H^{1}\left(C, \mu_{n}\right) \rightarrow \prod_{v \in \Omega} H^{1}\left(\kappa_{v}, \mu_{n}\right)$, où $\kappa_{v}$ désigne le corps résiduel en $v$.

(iii) Le groupe $H^{1}\left(\mathcal{C}, \mu_{n}\right)$.

(iv) Le groupe $H^{1}\left(\mathcal{C}_{0}, \mu_{n}\right)$.

(v) Le groupe $H^{1}\left(C_{0}, \mu_{n}\right)$.

Ils s'identifient tous à des sous-groupes de $H^{1}\left(C, \mu_{n}\right) \subset H^{1}\left(K, \mu_{n}\right)$.

Les groupes (i) et (ii) coïncident. Les groupes (iii), (iv), (v) coïncident. Le groupe (ii) est un sous-groupe du groupe (iii).

Si F est algébriquement clos, tous les groupes coïncident, et ce sont des groupes finis. 
Démonstration. - On a la suite exacte

$$
0 \rightarrow H^{1}\left(C, \mu_{n}\right) \rightarrow H^{1}\left(K, \mu_{n}\right) \rightarrow \bigoplus_{v \in C^{(1)}} \mathbb{Z} / n
$$

la suite exacte compatible

$$
0 \rightarrow H^{1}\left(O_{v}, \mu_{n}\right) \rightarrow H^{1}\left(K_{v}, \mu_{n}\right) \rightarrow \mathbb{Z} / n,
$$

et des isomorphismes

$$
H^{1}\left(O_{v}, \mu_{n}\right) \stackrel{\sim}{\rightarrow} H^{1}\left(\kappa_{v}, \mu_{n}\right) .
$$

Ceci établit l'isomorphisme entre (i) et (ii).

Comme $\mathcal{C}$ est régulier, le morphisme $\mathrm{Spec} K \hookrightarrow \mathcal{C}$ induit un isomorphisme $\mu_{n, \mathcal{C}} \stackrel{\simeq}{\rightarrow} i_{*} \mu_{n, K}$. On a donc des plongements

$$
H^{1}\left(\mathcal{C}, \mu_{n}\right) \hookrightarrow H^{1}\left(C, \mu_{n}\right) \hookrightarrow H^{1}\left(K, \mu_{n}\right) .
$$

Soit $\xi \in H^{1}\left(C, \mu_{n}\right)$ dans le groupe (ii). Soit $E \subset \mathcal{C}_{0}$ une composante intègre de la fibre spéciale $\mathcal{C}_{0}$. Soit $P$ un point fermé lisse de $E$ qui n'appartient à aucune autre composante de $\mathcal{C}_{0}$. Il existe un sous-schéma régulier intègre $Z \subset \mathcal{C}$, fini sur $\mathbb{C}[[t]]$, intersectant $E$ transversalement au point $P$. Soit $v \in C^{(1)}$ le point générique de $Z$. Les restrictions de $C$ à $v$ et de $E$ à $P$ induisent un diagramme commutatif pour les flèches résidus :

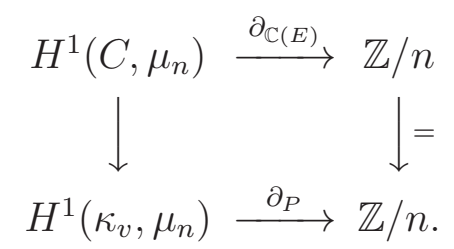

Comme $\xi$ a une image nulle dans $H^{1}\left(\kappa_{v}, \mu_{n}\right)$, le résidu de $\xi$ en $E$ est nul. Ainsi tous les résidus de $\xi$ aux points de codimension 1 du schéma régulier $\mathcal{C}$ sont nuls, ceci implique $\xi \in H^{1}\left(\mathcal{C}, \mu_{n}\right)$. Le groupe (ii) est donc dans le groupe (iii).

L'isomorphisme entre les groupes (iii) et (iv) est un cas spécial du théorème de changement de base propre ([23, VI, Cor. 2.7]).

L'isomorphisme entre les groupes (iv) et (v) : la cohomologie étale à coefficients $\mathbb{Z} / n$ est invariante par passage au sous-schéma réduit (cela vient de ce que si $X$ est un schéma, les petits sites étales de $X$ et $X_{\text {red }}$ sont isomorphes, ce qui résulte formellement de [23], Th. I.3.23).

L'adhérence d'un point $v$ de $C$ dans $\mathcal{C}$ est un schéma $Z_{v}$ intègre fini sur $F[[t]]$, hensélien, de corps des fractions $\kappa_{v}$. Supposons $F$ algébriquement clos. On a alors $H^{1}\left(Z, \mu_{n}\right)=0$. La flèche de restriction composée $H^{1}\left(\mathcal{C}, \mu_{n}\right) \rightarrow$ 
$H^{1}\left(Z, \mu_{n}\right) \rightarrow H^{1}\left(\kappa_{v}, \mu_{n}\right)$ est donc nulle. Ainsi le groupe (iii) est un sousgroupe du groupe (ii). Dans ce cas on a déjà que $H^{1}\left(C, \mu_{n}\right)$ est fini via la suite spectrale de Hochschild-Serre car le corps $k$ est à cohomologie galoisienne finie et $H^{1}\left(\bar{C}, \mu_{n}\right)$ est fini par [23], Th. VI.5.5.

Corollaire 2.4. - Soient $k=\mathbb{C}((t)), C$ une $k$-courbe projective, lisse, géométriquement intègre et $\mathcal{C} / \mathbb{C}[[t]]$ un modèle intègre régulier et propre de $C / k$. Soit $K$ le corps des fonctions de $C$. Soit $\mathcal{C}_{0}$ la fibre spéciale et $C_{0}$ la fibre spéciale réduite. Les groupes suivants s'identifient naturellement à un même sous-groupe fini de $H^{1}(K, \mathbb{Z} / n)$.

(i) Le groupe $\amalg^{1}(K, \mathbb{Z} / n)=\operatorname{Ker}\left[H^{1}(K, \mathbb{Z} / n) \rightarrow \prod_{v \in \Omega} H^{1}\left(K_{v}, \mathbb{Z} / n\right)\right]$.

(ii) Le noyau de la flèche d'évaluation $H^{1}(C, \mathbb{Z} / n) \rightarrow \prod_{v \in \Omega} H^{1}\left(\kappa_{v}, \mathbb{Z} / n\right)$, où $\kappa_{v}$ désigne le corps résiduel en $v$.

(iii) Le groupe $H^{1}(\mathcal{C}, \mathbb{Z} / n)$.

(iv) Le groupe $H^{1}\left(\mathcal{C}_{0}, \mathbb{Z} / n\right)$.

(v) Le groupe $H^{1}\left(C_{0}, \mathbb{Z} / n\right)$.

Corollaire 2.5. - Soient $k=\mathbb{C}((t)), C$ une $k$-courbe projective, lisse, géométriquement intègre. Le groupe $\amalg^{1}(K, \mathbb{Q} / \mathbb{Z})$ est un groupe divisible de type cofini.

Démonstration. - D'après le corollaire 2.4, le groupe $\amalg^{1}(K, \mathbb{Q} / \mathbb{Z})$ est isomorphe à $H^{1}\left(C_{0}, \mathbb{Q} / \mathbb{Z}\right)$, où $C_{0}$ est une courbe propre sur $\mathbb{C}$. Il suffit alors d'utiliser la proposition bien connue suivante :

Soit $C$ une courbe propre sur un corps algébriquement clos de caractéristique zéro. Alors le groupe $H^{1}(C, \mathbb{Q} / \mathbb{Z})$ est un groupe divisible.

Faute de référence, nous indiquons une preuve de cette proposition. On peut supposer $C$ réduite. Soit $\pi: \widetilde{C} \rightarrow C$ la normalisation. On dispose d'une suite exacte de faisceaux

$$
0 \rightarrow \mathbb{Q} / \mathbb{Z} \rightarrow \pi_{*}(\mathbb{Q} / \mathbb{Z}) \rightarrow \bigoplus_{m \in S}\left(i_{m}\right)_{*} F_{m} \rightarrow 0
$$

où $S$ est un ensemble fini de points de $C$ et où chaque $F_{m}$ est une somme directe d'exemplaires de $\mathbb{Q} / \mathbb{Z}$. On en déduit une suite exacte

$$
\bigoplus F_{m} \rightarrow H^{1}(C, \mathbb{Q} / \mathbb{Z}) \rightarrow H^{1}(\tilde{C}, \mathbb{Q} / \mathbb{Z}) \rightarrow 0 .
$$

La courbe $\tilde{C}$ est une union disjointe de courbes propres et lisses, pour une telle courbe $D$, le groupe $H^{1}(D, \mathbb{Q} / \mathbb{Z})$ s'identifie au groupe des points de torsion de la jacobienne, qui est un groupe divisible. 
Pour tout module galoisien $M$ et tout $i>0$, on notera $\amalg_{\omega}^{i}(K, M)$ (ou simplement $\amalg_{\omega}^{i}(M)$ si $K$ est sous-entendu) le sous-groupe de $H^{i}(K, M)$ des éléments d'image nulle dans tous les $H^{i}\left(K_{v}, M\right)$ sauf peut-être un nombre fini.

Proposition 2.6. - Soit $k=\mathbb{C}((t))$.

(i) On a $\amalg^{1}\left(K, \mu_{n}\right)=\amalg_{\omega}^{1}\left(K, \mu_{n}\right)$.

(ii) On a $\amalg^{1}(K, \mathbb{Z} / n)=\amalg_{\omega}^{1}(K, \mathbb{Z} / n)$.

(iii) On a $\amalg^{1}(K, \mathbb{Q} / \mathbb{Z})=\amalg_{\omega}^{1}(K, \mathbb{Q} / \mathbb{Z})$, et ce groupe est divisible.

(iv) Pour tout $\operatorname{Gal}(\bar{K} / K)$-module de permutation $P$, on a $\amalg^{2}(K, P)=$ $\amalg_{\omega}^{2}(K, P)$, et ce groupe est divisible.

Démonstration. - L'énoncé (i) implique clairement (ii) et (iii), la divisibilité venant du corollaire 2.5. Soit $L / K$ une extension finie de corps, et $G_{L} \subset$ $G_{K}$ les groupes de Galois absolus. Soit $P=\mathbb{Z}\left[G_{K} / G_{L}\right]$. On a $H^{2}(K, P)=$ $H^{2}(L, \mathbb{Z})$ et l'énoncé analogue sur tout complété $K_{v}$ de $K$. On a donc

$$
\amalg_{\omega_{K}}^{2}(K, P)=\amalg_{\omega_{L}}^{2}(L, \mathbb{Z})=\amalg_{\omega_{L}}^{1}(L, \mathbb{Q} / \mathbb{Z})=\amalg^{1}(L, \mathbb{Q} / \mathbb{Z})=\amalg^{2}(K, P)
$$

par application du résultat (iii) au corps $L$, qui est le corps des fonctions d'une courbe sur un corps extension finie de $\mathbb{C}((t))$, donc isomorphe à $\mathbb{C}((t))$. La divisibilité du groupe considéré suit aussi de (iii).

Il suffit donc d'établir l'énoncé (i).

On a $H^{1}\left(K, \mu_{n}\right)=K^{\times} / K^{\times n}$ et $H^{1}\left(K_{v}, \mu_{n}\right)=K_{v}^{\times} / K_{v}^{\times n}$. Soit $f \in K^{\times}$ dont l'image dans $H^{1}\left(K, \mu_{n}\right)$ est dans $\amalg_{\omega}^{1}\left(K, \mu_{n}\right)$. Supposons d'abord $v(f)$ nul modulo $n$ pour toute place $v \in C^{(1)}$. Alors le revêtement $D:=C(\sqrt[n]{f})$ de $C$ est étale. Pour toute extension finie $k^{\prime}$ de $k$, l'image de l'application $D\left(k^{\prime}\right) \rightarrow C\left(k^{\prime}\right)$ est fermée par propreté de $D \rightarrow C$, et le complémentaire de cette image dans $C\left(k^{\prime}\right)$ est fini car par hypothèse, le revêtement $D \rightarrow C$ est totalement décomposé en presque toute place $v \in C^{(1)}$. On en déduit que $D\left(k^{\prime}\right) \rightarrow C\left(k^{\prime}\right)$ est surjectif, et le revêtement $D \rightarrow C$ étant étale, ceci implique qu'il est totalement décomposé en toute place $v \in C^{(1)}$, i.e. la classe de $f$ est dans $\amalg^{1}\left(K, \mu_{n}\right)$.

Supposons désormais qu'il existe $v \in C^{(1)}$ avec $v(f)$ non nul modulo $n$. Soit $P$ le point fermé de $C$ défini par $v$ et soit $L=\kappa_{v}$ son corps résiduel.

Soit $\pi \in K^{\times}$une uniformisante de $C_{L}$ en $P$. On a donc $f=u . \pi^{r}$ avec $r \neq 0 \bmod n$ et $u \in K^{\times}$unité en $P$.

Par le théorème des fonctions implicites sur le corps $L$, il existe un voisinage ouvert analytique $U \subset C(L)$ de $P$ et un ouvert $V \subset L$ contenant 0 
tel que $\pi$ induise un isomorphisme entre $U$ et $V$. Pour $U$ suffisamment petit, et $Q \in U, Q \neq P$, la classe de $u(Q)$ dans $L^{\times} / L^{\times n} \stackrel{\widetilde{\sim}}{\rightarrow} \mathbb{Z} / n$ est constante, et la classe de $\pi(Q)$ prend toutes les valeurs dans $L^{\times} / L^{\times n} \stackrel{\widetilde{T}}{\rightarrow} / n$. Il existe donc une infinité de points $Q \in C(L)$, de valuation associée $v=v_{Q}$, tels que $v(f(Q)) \neq 0 \bmod n$, et donc $f \notin K_{v}^{\times} / K_{v}^{\times n}$. Pour conclure que toute classe dans $\amalg_{\omega}^{1}\left(K, \mu_{n}\right)$ appartient à $H^{1}\left(C, \mu_{n}\right)$, il suffit de montrer qu' on peut trouver une infinité de points $Q$ comme ci-dessus qui de plus définissent des points fermés de $C$. Or, pour $U$ suffisamment petit, $U \backslash P$ est une union finie de $n$ ouverts non vides $U_{i}, i=1, \ldots, n$ tels que sur $U_{i}$ on ait $v_{L}(\pi(Q))=i \in \mathbb{Z} / n$. On conclut avec le lemme suivant :

Lemme 2.7. - Soient $K$ un corps complet pour une valuation discrète et $L / K$ une extension finie séparable. Soit $X$ une $K$-variété lisse intègre. Dans $X(L)$, la réunion des $X(F) \subset X(L)$ pour $F / K$ sous-extension propre de $L / K$ est un fermé dont le complémentaire est dense dans $X(L)$.

Le lemme se montre facilement en se ramenant (via le théorème des fonctions implicites) au cas où $X$ est l'espace affine, en prenant un ouvert de Zariski non vide de $X$ équipé d'un morphisme étale vers $\mathbf{A}_{K}^{n}$.

Remarques 2.8. - (1) Soit $k=\mathbb{C}((t))$ et $C$ une $k$-courbe projective, lisse, géométriquement intègre d'indice $I=I(C)>1$. Alors l'application composée

$$
H^{1}(k, \mathbb{Z} / I) \rightarrow H^{1}(C, \mathbb{Z} / I) \rightarrow \prod_{v \in \Omega} H^{1}\left(\kappa_{v}, \mathbb{Z} / I\right)
$$

est nulle, et l'application composée

$$
H^{1}(k, \mathbb{Z} / I) \rightarrow H^{1}(C, \mathbb{Z} / I) \rightarrow H^{1}(K, \mathbb{Z} / I)
$$

est injective. On a donc dans ce cas

$$
\mathbb{Z} / I \simeq H^{1}(k, \mathbb{Z} / I) \subset \amalg^{1}(K, \mathbb{Z} / I) \subset \amalg^{1}(K, \mathbb{Q} / \mathbb{Z}) .
$$

(2) Un exemple concret est fourni par la courbe $C$ d'équation

$$
X^{3}+t Y^{3}+t^{2} Z^{3}=0 .
$$

On a $I(C)=3$. La classe de $t \in k^{\times} / k^{\times 3}$ s'annule dans tout $\kappa_{v}^{\times} / \kappa_{v}^{\times 3}$, c'est un élément non nul de $\amalg^{1}(\mathbb{Z} / 3)$. Le modèle régulier minimal de $C$ a une fibre spéciale de type $3 I_{0}$. La courbe $C_{0}$ étant une courbe elliptique (lisse) sur $\mathbb{C}$, le corollaire 2.4 donne donc $\amalg^{1}(K, \mathbb{Z} / 3)=(\mathbb{Z} / 3)^{2}$. 
(3) Comme dans [6], on peut considérer le sous-groupe

$$
\amalg_{\text {total }}^{1}(K, \mathbb{Z} / n)=\operatorname{Ker}\left[H^{1}(K, \mathbb{Z} / n) \rightarrow \prod_{v \in \mathcal{C}^{(1)}} H^{1}\left(K_{v}, \mathbb{Z} / n\right)\right]
$$

de $\amalg^{1}(K, \mathbb{Z} / n)$. Il s'identifie au groupe des éléments de $H^{1}\left(C_{0}, \mathbb{Z} / n\right)$ qui s'annulent au point générique de chaque composante connexe de $C_{0}$. On peut aussi vérifier qu'il s'identifie au groupe des éléments de $H^{1}(K, \mathbb{Z} / n)$ dont la restriction à tout complété de $K$ en une valuation discrète de rang 1 quelconque de $K$ est nulle.

Le groupe $\Psi_{\text {total }}^{1}(K, \mathbb{Z} / n)$ est nul si la fibre spéciale réduite $C_{0}$ est lisse, car $H^{1}\left(C_{0}, \mathbb{Z} / n\right) \rightarrow H^{1}\left(\mathbb{C}\left(C_{0}\right), \mathbb{Z} / n\right)$ est alors injectif. Ceci s'applique à l'exemple (2) ci-dessus.

Si chaque composante de $C_{0}$ est une courbe lisse de genre zéro, les applications de restriction de $H^{1}\left(C_{0}, \mathbb{Z} / n\right)$ au point générique de chaque composante se factorisent par $H^{1}\left(\mathbf{P}_{\mathbb{C}}^{1}, \mathbb{Z} / n\right)=0$, on a alors $\amalg_{\text {total }}^{1}(K, \mathbb{Z} / n)=$ $H^{1}\left(C_{0}, \mathbb{Z} / n\right)$. Un exemple avec $\amalg_{\text {total }}^{1}(K, \mathbb{Z} / n)$ non nul est donc fourni par toute courbe elliptique $C / k$ dont la fibre spéciale est de type $I_{m}$ avec $m \geq 2$. Comparer avec les paragraphes 7 et 8 (et particulièrement le corollaire 8.1) de [19].

(4) $\mathrm{Si} K$ est un corps de nombres, le théorème de Tchebotarev donne $\amalg^{1}(K, \mathbb{Z} / n)=\amalg_{\omega}^{1}(K, \mathbb{Z} / n)=0$, et de même avec $\mathbb{Q} / \mathbb{Z}$ au lieu de $\mathbb{Z} / n$. On a donc aussi dans ce cas $\amalg_{\omega}^{2}(K, P)=\amalg^{2}(K, P)=0$ pour tout module de permutation $P$. Par contre $\amalg_{\omega}^{1}\left(K, \mu_{n}\right)$ peut contenir strictement $\amalg^{1}\left(K, \mu_{n}\right)$, c'est par exemple le cas pour $K=\mathbb{Q}$ et $n=8$ (Grunwald-Wang).

\subsection{Le groupe $\amalg^{2}\left(K, \mu_{n}\right)$. -}

Proposition 2.9. - Soient $k=\mathbb{C}((t)), C$ une $k$-courbe projective, lisse, géométriquement intègre et $\mathcal{C} / \mathbb{C}[[t]]$ un modèle intègre régulier et propre de $C / k$. Soit $K$ le corps des fonctions de $C$. Soit $\mathcal{C}_{0}$ la fibre spéciale et $C_{0}$ la fibre spéciale réduite. Les groupes suivants s'identifient naturellement à un même sous-groupe fini de $H^{2}\left(K, \mu_{n}\right)$.

(i) Le groupe $\amalg^{2}\left(K, \mu_{n}\right)=\operatorname{Ker}\left[H^{2}\left(K, \mu_{n}\right) \rightarrow \prod_{v \in \Omega} H^{2}\left(K_{v}, \mu_{n}\right)\right]$.

(ii) Le groupe ${ }_{n} \operatorname{Br}(C)$.

(iii) Le groupe $H_{C_{0}}^{3}\left(\mathcal{C}, \mu_{n}\right)$.

(iv) Le groupe $\operatorname{Hom}\left(H^{1}\left(C_{0}, \mu_{n}\right), \mathbb{Z} / n\right)$.

(v) Le groupe $\operatorname{Hom}\left(\amalg^{1}\left(K, \mu_{n}\right), \mathbb{Z} / n\right)$.

Démonstration. - On a $H^{2}\left(K, \mu_{n}\right)={ }_{n} \operatorname{Br} K$ et $H^{2}\left(K_{v}, \mu_{n}\right)={ }_{n} \operatorname{Br} K_{v}$; la proposition 2.1(v) montre que les groupes (i) et (ii) coïncident. 
On a $\mathrm{BrC} \stackrel{\simeq}{\rightrightarrows} \mathrm{Br}_{0}=0$ (Artin, Grothendieck [13, III, Thm. 3.1 et Cor. 5.8]). Comme $\mathcal{C}$ est régulier, la restriction $\operatorname{Pic} \mathcal{C} \rightarrow \operatorname{Pic} C$ est surjective. De la suite exacte de localisation pour la cohomologie à support

$$
H^{2}\left(\mathcal{C}, \mu_{n}\right) \rightarrow H^{2}\left(C, \mu_{n}\right) \rightarrow H_{C_{0}}^{3}\left(\mathcal{C}, \mu_{n}\right) \rightarrow H^{3}\left(\mathcal{C}, \mu_{n}\right)
$$

et de la suite de Kummer pour $\mathcal{C}$ et pour $C$ on déduit donc une suite exacte

$$
0 \rightarrow{ }_{n} \operatorname{Br}(C) \rightarrow H_{C_{0}}^{3}\left(\mathcal{C}, \mu_{n}\right) \rightarrow H^{3}\left(\mathcal{C}, \mu_{n}\right) .
$$

Le théorème de changement de base propre donne

$$
H^{3}\left(\mathcal{C}, \mu_{n}\right) \stackrel{\simeq}{\rightarrow} H^{3}\left(C_{0}, \mu_{n}\right)=0 .
$$

On a donc ${ }_{n} \operatorname{Br}(C) \stackrel{\simeq}{\rightarrow} H_{C_{0}}^{3}\left(\mathcal{C}, \mu_{n}\right)$. L'isomorphisme entre les groupes (iii) et (iv) est un cas particulier d'un théorème général de dualité qu'on peut trouver explicité dans [9, Prop. 2.6].

L'identification entre le groupe (iv) et le groupe (v) résulte alors de la proposition 2.3

Exemple 2.10. - D'après les propositions 2.1 et 2.9, on a les égalités ${ }_{n} \mathrm{BrC}=\operatorname{Ker}\left[{ }_{n} \operatorname{Br} K \rightarrow \prod_{v}{ }_{n} \operatorname{Br} K_{v}\right]$ et ${ }_{n} \operatorname{Br}(C)=\operatorname{Hom}\left(H^{1}\left(C_{0}, \mu_{n}\right), \mathbb{Z} / n\right)$. Supposons $H^{1}\left(C_{0}, \mu_{n}\right) \neq 0$. Soit $\alpha \neq 0$ dans $\operatorname{Ker}\left[{ }_{n} \operatorname{Br} K \rightarrow \prod_{v}{ }_{n} \operatorname{Br} K_{v}\right]$. Il existe une extension finie $L / K$ de corps, avec $\alpha_{L}=0 \in \operatorname{Br} L$, extension qu'on peut prendre de degré divisant $n$, car pour toute algèbre simple centrale $D$ sur $K$, indice et exposant de $D$ coïncident, comme rappelé au $\$ 1$. Le plongement naturel $\mathbb{G}_{m, K} \rightarrow R_{L / K} \mathbb{G}_{m, L}$ induit une suite exacte de $K$-tores algébriques

$$
1 \rightarrow \mathbb{G}_{m, K} \rightarrow R_{L / K} \mathbb{G}_{m, L} \rightarrow T \rightarrow 1
$$

Les suites de cohomologie associées sur $K$ et sur les $K_{v}$, le théorème de Hilbert 90 et le lemme de Shapiro donnent une suite exacte

$$
0 \rightarrow \amalg^{1}(K, T) \rightarrow \amalg^{2}\left(K, \mathbb{G}_{m}\right) \rightarrow \amalg^{2}\left(L, \mathbb{G}_{m}\right) .
$$

On a donc $\amalg^{1}(K, T) \neq 0$. On notera que le $K$-tore $T$ est $K$-birationnel à l'espace projectif. La situation ici est donc différente de celle qui vaut sur les corps globaux ([28], Cor. 9.7) ou sur les corps de fonctions d'une variable sur les corps $p$-adiques ([17], Cor. 5.7).)

Il est facile de donner un exemple numérique. On part de la courbe elliptique constante $E$ donnée par l'équation affine

$$
y^{2}=x(x-1)(x+1),
$$

et on note $C=E \times_{\mathbb{C}} \mathbb{C}((t))$. La classe de quaternions $\alpha=(x, t)$ non ramifiée sur $C$ est nulle dans chaque $K_{v}$ mais pas dans $K$. On peut prendre alors pour 
$T$ soit $X^{2}-x Y^{2}=1$ (avec l'espace principal homogène $X^{2}-x Y^{2}=t$ ), soit $X^{2}-t Y^{2}=1$ (avec l'espace principal homogène $X^{2}-t Y^{2}=x$ ).

\section{Dualité locale pour la cohomologie des groupes finis et des tores}

Dans ce paragraphe, on note $K=\mathbb{C}((t))((u))$. Toute extension finie $L$ de $K$ est de la forme $\mathbb{C}\left(\left(t^{\prime}\right)\right)\left(\left(u^{\prime}\right)\right)$.

Lemme 3.1. - Soit $S$ un schéma. Soit $\mathcal{T}$ un tore sur $S$ et $\widehat{\mathcal{T}}$ son groupe des caractères. Alors on a un diagramme commutatif (dans la catégorie dérivée bornée des faisceaux fppf sur $S$ ) :

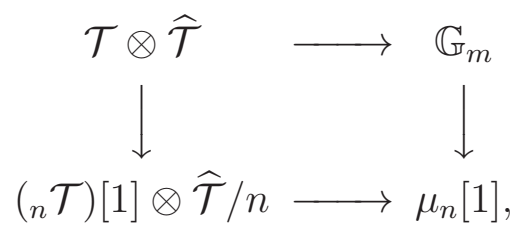

où la flèche $\mathbb{G}_{m} \rightarrow \mu_{n}[1]$ est induite par le triangle exact

$$
\mu_{n} \rightarrow \mathbb{G}_{m} \stackrel{. n}{\rightarrow} \mathbb{G}_{m} \rightarrow \mu_{n}[1] .
$$

Démonstration. - On part du triangle exact

$$
{ }_{n} \mathcal{T} \rightarrow \mathcal{T} \stackrel{{ }_{n}}{\rightarrow} \mathcal{T} \rightarrow\left({ }_{n} \mathcal{T}\right)[1],
$$

que l'on peut tensoriser par le faisceau $\widehat{\mathcal{T}}$ (qui est localement isomorphe à $\mathbb{Z}^{r}$ pour la topologie étale, avec $r \geq 0$ ). Comme $\widehat{\mathcal{T}}=\operatorname{Hom}\left(\mathcal{T}, \mathbb{G}_{m}\right)$, on en déduit le résultat en observant que

$$
\left({ }_{n} \mathcal{T}\right)[1] \otimes \widehat{\mathcal{T}}=\left({ }_{n} \mathcal{T}\right)[1] \otimes \widehat{\mathcal{T}} / n
$$

et $\widehat{\mathcal{T}} / n=\operatorname{Hom}\left({ }_{n} \mathcal{T}, \mathbb{G}_{m}\right)=\operatorname{Hom}\left({ }_{n} \mathcal{T}, \mu_{n}\right)$.

Lemme 3.2. - (i) Pour tout L-groupe abélien fini $M$, et tout entier $i \geq 0$, le groupe $H^{i}(L, M)$ est fini, et nul pour $i \geq 3$.

(ii) Pour tout $L$-groupe de type multiplicatif $M$ sur $L$, le groupe $H^{1}(L, M)$ est fini.

Démonstration. - (i) Soit $L^{\prime} / L$ une extension finie galoisienne déployant $M$. La finitude résulte via la suite spectrale de Hochschild-Serre de la nullité de $H^{i}\left(L^{\prime}, \mu_{n}\right)$ pour $i \geq 3$ et de la finitude des groupes $H^{1}\left(L^{\prime}, \mu_{n}\right)$ ainsi que $H^{2}\left(L^{\prime}, \mu_{n}\right)={ }_{n} \operatorname{Br} L^{\prime}$. 
Proposition 3.3. - Soit F un module galoisien fini, de dual de Cartier $F^{0}=$ $\operatorname{Hom}(F, \mathbb{Q} / \mathbb{Z}(1))$. Pour $0 \leq i \leq 2$, on a des dualités parfaites de groupes finis

$$
H^{i}(K, F) \times H^{2-i}\left(K, F^{0}\right) \rightarrow H^{2}(K, \mathbb{Q} / \mathbb{Z}(1))=\mathbb{Q} / \mathbb{Z}(-1) .
$$

Démonstration. - [29, §5.1, Exercice 2].

Pour $T$ un $K$-tore, et $\widehat{T}=\operatorname{Hom}\left(T, \mathbb{G}_{m, K}\right)$, on définit un accouplement

$$
H^{i}(K, T) \times H^{2-i}(K, \widehat{T}) \rightarrow H^{2}\left(K, \mathbb{G}_{m}\right)=\mathbb{Q} / \mathbb{Z}(-1) .
$$

Proposition 3.4. - Pour $i=1$, l'accouplement ci-dessus est un accouplement parfait de groupes finis. Pour $i=0$, il induit une dualité parfaite entre le complété profini de $H^{0}(K, T)$ et le groupe discret $H^{2}(K, \widehat{T})$, et pour $i=2$ une dualité parfaite entre le groupe discret $H^{2}(K, T)$ et le complété profini de $H^{0}(K, \widehat{T})$.

Démonstration. - Notons $A^{D}:=\operatorname{Hom}(A, \mathbb{Q} / \mathbb{Z}(-1))$. Pour tout $n>0$, on a un diagramme commutatif (via le lemme 3.1) de groupes finis, à lignes exactes

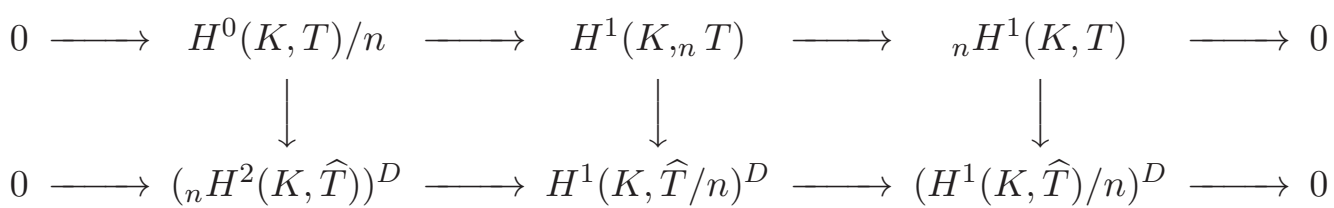

La flèche verticale du milieu est un isomorphisme d'après la Proposition 3.3 . Comme $H^{1}(K, T)$ et $H^{1}(K, \widehat{T})$ sont finis (ils sont de type cofini, et d'exposant fini via Hilbert 90 et $H^{1}(K, \mathbb{Z})=0$ ), on en déduit en prenant $n$ convenable une flèche surjective $H^{1}(K, T) \rightarrow H^{1}(K, \widehat{T})^{D}$. On écrit alors un autre diagramme commutatif de groupes finis, à lignes exactes

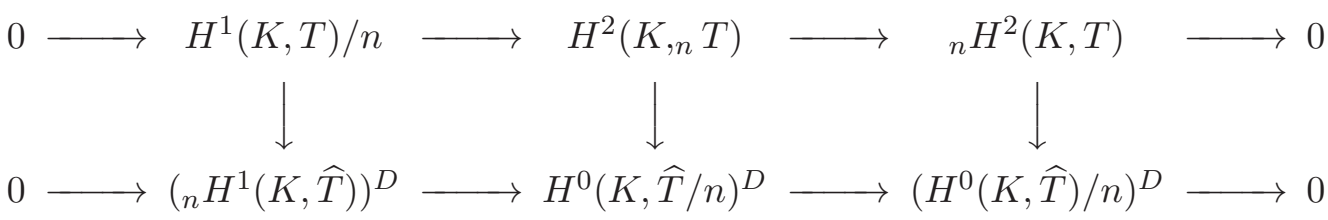

dont la flèche verticale du milieu est un isomorphisme d'après la Proposition 3.3. En prenant encore $n$ convenable, on en déduit une flèche injective $H^{1}(K, T) \rightarrow H^{1}(K, \widehat{T})^{D}$, ce qui par cardinalité donne finalement que l'accouplement entre $H^{1}(K, T)$ est $H^{1}(K, \widehat{T})$ est un accouplement parfait de groupes finis. 
Les deux diagrammes donnent alors que les deux flèches $T(K) / n \rightarrow$ $\left({ }_{n} H^{2}(K, \widehat{T})\right)^{D}$ et $H^{2}(K, T)_{n} \rightarrow\left(H^{0}(K, \widehat{T}) / n\right)^{D}$ sont également des isomorphismes, ce qui achève la preuve de la proposition en passant à la limite projective (resp. inductive) sur $n$.

Remarque 3.5. - L'application naturelle de $T(K)$ équipé de la topologie induite par celle de $K$, vers le complété profini de $T(K)$ (qui est aussi la limite projective des $T(K) / n$ pour $n>0$ entier vu que chaque $T(K) / n$ est fini) est continue. L'application qui va de $T(K)$ vers le dual du groupe discret $H^{2}(K, \widehat{T})$ est donc aussi continue.

De la proposition ci-dessus on déduit l'énoncé suivant, analogue d'un résultat connu sur les corps $p$-adiques.

Corollaire 3.6. - Soit $K=\mathbb{C}((u))((t))$. Pour tout $K$-tore coflasque $Q$, on a $H^{1}(K, Q)=0$. En d'autre termes, tout espace principal homogène sous un tel $K$-tore possède un point rationnel.

Démonstration. - En effet par définition, un $K$-tore est coflasque s'il satisfait l'égalité $H^{1}(L, \widehat{Q})=0$ pour toute extension finie de corps $L / K$.

\section{Cohomologie des courbes : localisation et finitude}

On commence par quelques rappels généraux.

Proposition 4.1. - Soit A un anneau semilocal régulier intègre de corps des fractions $K$. Pour tout A-groupe de type multiplicatif lisse $G$, et tout entier $i=0,1,2$, les applications de restriction $H^{i}(A, G) \rightarrow H^{i}\left(K, G_{K}\right)$ sont injectives.

Démonstration. - C'est la proposition 2.2 page 450 de [7], laquelle vaut plus généralement pour un anneau semilocal géométriquement localement factoriel.

Proposition 4.2. - Soit $X$ un schéma de Dedekind intègre de corps des fractions $K$. Soit $U \subset X$ un ouvert non vide.

(i) Soit $M$ un $X$-schéma en groupes finis abéliens étale d'ordre premier aux caractéristiques résiduelles de X. Si une classe dans $H^{i}(U, M)$ a une image nulle dans tous les $H^{i}\left(K_{v}, M\right)$ pour $v \notin U$, alors elle est dans l'image de la restriction $H^{i}(X, M) \rightarrow H^{i}(U, M)$. 
(ii) Soit $M$ un $X$-schéma en groupes réductifs. Si une classe de $H^{1}(U, M)$ a une image nulle dans tous les $H^{1}\left(K_{v}, M\right)$ pour $v \notin U$, alors elle est dans l'image de la restriction $H^{1}(X, M) \rightarrow H^{1}(U, M)$.

Démonstration. - Pour (i), il suffit de considérer la suite de localisation pour la cohomologie étale de $X$, qui est fonctorielle, et le théorème de pureté pour la cohomologie sur un anneau de valuation discrète ([2], section 3.3). On peut ici utiliser soit les hensélisés soit les complétés. Pour (ii), voir [21, Lemma 4.1.3] ou [10, Cor. 4.8].

Dans la suite de ce paragraphe, on considère $C$ une courbe intègre, projective et lisse sur un corps de caractéristique zéro $k$, et $U \subset C$ un ouvert de Zariski non vide. On note $K=k(C)$.

Comme $C$ est une courbe régulière, pour tout faisceau étale $\mathcal{F}$ sur $U$ (dont on note $F$ la restriction à la fibre générique), on a pour $i \geq 0$ de longues suites exactes [24, II, Prop. 2.3] :

$$
\cdots \rightarrow H_{c}^{i}(U, \mathcal{F}) \rightarrow H^{i}(U, \mathcal{F}) \rightarrow \bigoplus_{v \notin U} H^{i}\left(K_{v}^{h}, F\right) \rightarrow H_{c}^{i+1}(U, \mathcal{F}) \ldots
$$

où $K_{v}^{h}=\operatorname{Frac} \mathcal{O}_{v}^{h}$ est le corps des fractions du hensélisé $\mathcal{O}_{v}^{h}$ de l'anneau local de $C$ en $v$ (pour simplifier les notations, on notera souvent $H^{i}\left(K_{v}^{h}, F\right)$ pour $H^{i}\left(K_{v}^{h}, F_{v}\right)$, où $F_{v}$ est la restriction de $F$ à $\left.K_{v}^{h}\right)$. Si $i \geq 1$ et $F$ est représenté par un $K$-schéma en groupes localement de type fini (par exemple un tore, un $K$-groupe fini, ou le module des caractères d'un tore), on peut remplacer $K_{v}^{h}$ par le complété $K_{v}$ via le théorème d'approximation de Greenberg (cf. [15], Lemme 2.7 pour un argument détaillé). Les propriétés de fonctorialité de la cohomologie à support compact sont résumées dans la proposition suivante, dont certains points sont déjà discutés dans la Prop. 4.2. de [17] :

Proposition 4.3. - Avec les notations ci-dessus, on considère deux ouverts de Zariski non vides $U$ et $V$ de $C$ avec $V \subset U$. Soit $\mathcal{F}$ un faisceau étale sur $U$. Soit $i \geq 0$ un entier.

a) L'inclusion $V \subset U$ induit une flèche $H_{c}^{i}(V, \mathcal{F}) \rightarrow H_{c}^{i}(U, \mathcal{F})$ telles que si $W \subset V$, les flèches

$$
H_{c}^{i}(W, \mathcal{F}) \rightarrow H_{c}^{i}(V, \mathcal{F}) \rightarrow H_{c}^{i}(U, \mathcal{F})
$$

soient compatibles. 
b) Le diagramme

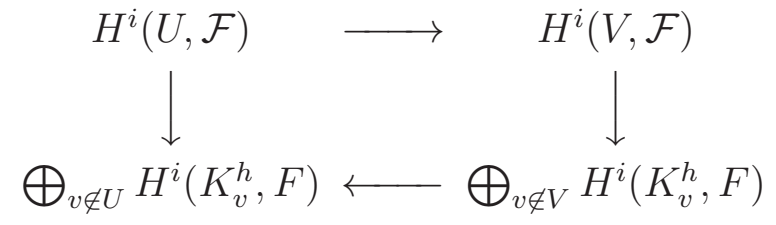

est commutatif, où la flèche horizontale du bas est la projection.

c) Le diagramme

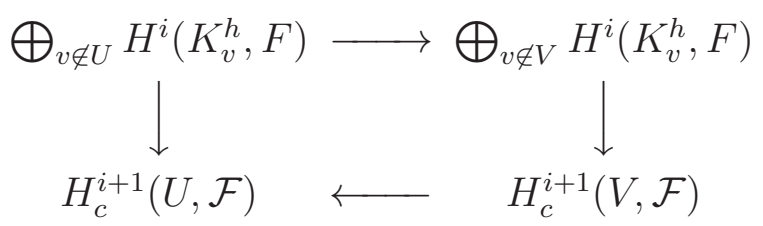

est commutatif, où la flèche horizontale du haut est l'injection canonique $\left(f_{v}\right) \mapsto\left(\left(f_{v}\right), 0,0, \ldots, 0\right)$.

d) Le diagramme

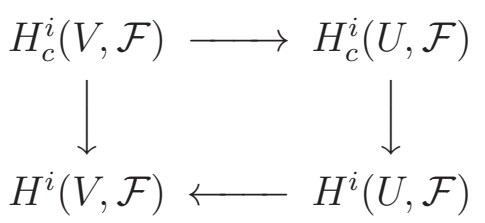

est commutatif. En particulier si on pose

$$
\mathcal{D}^{i}(U, \mathcal{F}):=\operatorname{Im}\left[H_{c}^{i}(U, \mathcal{F}) \rightarrow H^{i}(K, F)\right],
$$

on a $\mathcal{D}^{i}(V, \mathcal{F}) \subset \mathcal{D}^{i}(U, \mathcal{F})$.

e) Pour $i \geq 1$, on a un complexe

$$
\bigoplus_{v \in C^{(1)}} H^{i-1}\left(K_{v}^{h}, F\right) \rightarrow H_{c}^{i}(U, \mathcal{F}) \rightarrow H^{i}(K, F)
$$

qui est de plus exact si pour toute place $v \in U$, la flèche $H^{i}\left(\mathcal{O}_{v}^{h}, \mathcal{F}\right) \rightarrow$ $H^{i}\left(K_{v}^{h}, F\right)$ est injective, par exemple si $\mathcal{F}$ est représenté par un schéma en groupes fini étale, ou pour $i \leq 2$ si $\mathcal{F}$ est représenté par un schéma en groupes de type multiplicatif.

f) Soit $\mathcal{F}$ un groupe de type multiplicatif sur $U$ de dual $\mathcal{F}^{\prime}=\operatorname{Hom}\left(\mathcal{F}, \mathbb{G}_{m}\right)$. On note $F$ et $F^{\prime}$ les fibres génériques sur $\operatorname{Spec} K$ de $F$ et $F^{\prime}$. Soit $v \in C^{(1)}$. Alors pour tous entiers $i, j \geq 0$, on a un diagramme commutatif: 


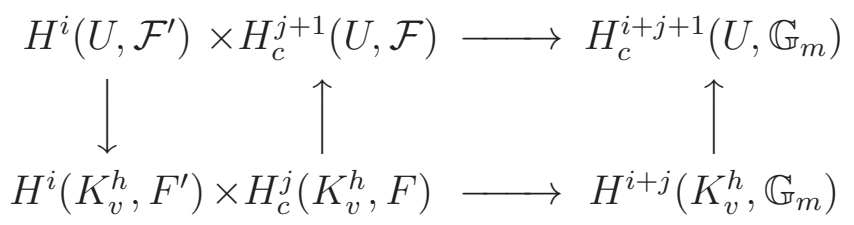

et de même avec $\mathcal{F}$ et $\mathcal{F}^{\prime}$ échangés.

Noter que dans ces énoncés, on pourra souvent remplacer les hensélisés $K_{v}^{h}$ par les complétés $K_{v}$, comme on l'a expliqué plus haut.

Démonstration. - a) résulte de la définition de $H_{c}^{i}$ et du fait que les immersions ouvertes $W \subset V \subset U$ induisent des morphismes compatibles de faisceaux $\left(j_{W}\right) ! \mathcal{F} \rightarrow\left(j_{V}\right) ! \mathcal{F} \rightarrow\left(j_{U}\right) ! \mathcal{F}$.

b) vient de ce que pour toute place $v$, la flèche $H^{i}(U, \mathcal{F}) \rightarrow H^{i}\left(K_{v}^{h}, F\right)$ est la composée des restrictions $H^{i}(U, \mathcal{F}) \rightarrow H^{i}(K, F)$ et $H^{i}(K, F) \rightarrow$ $H^{i}\left(K_{v}^{h}, F\right)$.

Pour démontrer c), il suffit (par linéarité) de prouver que si $v \in C^{(1)}$ n'est pas dans $U$ (et donc pas non plus dans $V$ ), le triangle

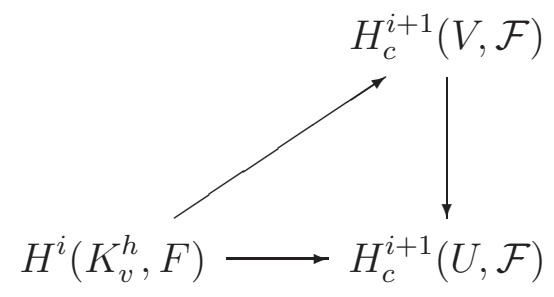

est commutatif. Par ([24], Prop. 1.1), on a $H^{i}\left(K_{v}^{h}, F\right)=H_{v}^{i+1}\left(\mathcal{O}_{v}^{h},\left(j_{v}\right) ! F_{v}\right)$, où $F_{v}=F_{\mid K_{v}^{h}}$ et $j_{v}: \operatorname{Spec} K_{v}^{h} \rightarrow \operatorname{Spec} \mathcal{O}_{v}^{h}$ est l'inclusion. Par ailleurs, la flèche $H^{i}\left(K_{v}^{h}, F\right) \rightarrow H_{c}^{i+1}(U, \mathcal{F})$ est induite (cf. [24], Lemme 2.4) par la flèche $H_{v}^{i+1}\left(C,\left(j_{U}\right) ! \mathcal{F}\right) \rightarrow H^{i+1}\left(C,\left(j_{U}\right)_{!} \mathcal{F}\right)=H_{c}^{i+1}(U, \mathcal{F})$ (qui vient de la suite de localisation) et par l'identification de $H_{v}^{i+1}\left(C,\left(j_{U}\right) ! \mathcal{F}\right)$ avec $H_{v}^{i+1}\left(\mathcal{O}_{v}^{h},\left(j_{v}\right)_{!} F_{v}\right)$ (excision, [23], Cor. III.1.28). La même chose est valable pour $V$, et on conclut alors la preuve de c) via la commutativité du diagramme

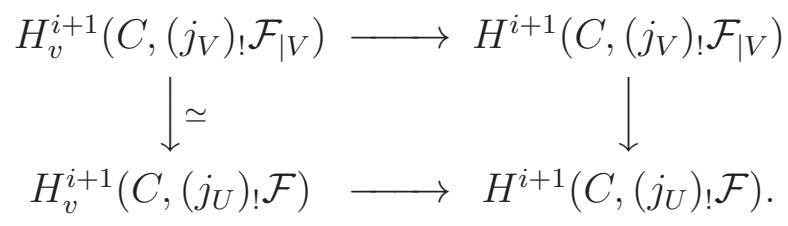

d) est démontré dans [17], Prop. 3.1. (3). 
La preuve de e) est identique à celle de la Prop. 4.2. de [17], en rappelant que pour toute place $v \in C^{(1)}$, la flèche $H^{i-1}\left(K_{v}^{h}, F\right) \rightarrow H_{c}^{i}(U, \mathcal{F})$ est définie en choisissant un ouvert non vide $V \subset U$ tel que $v \notin V$, puis en composant la flèche $H^{i-1}\left(K_{v}^{h}, F\right) \rightarrow H_{c}^{i}(V, \mathcal{F})$ (qui vient de la suite exacte (4.1)) avec la flèche $H_{c}^{i}(V, \mathcal{F}) \rightarrow H_{c}^{i}(U, \mathcal{F})$ provenant de l'inclusion $V \subset U$; cette définition ne dépend pas de $V$ d'après c).

Il reste juste à vérifier que dans les deux cas particuliers considérés, la flèche $H^{i}\left(\mathcal{O}_{v}^{h}, \mathcal{F}\right) \rightarrow H^{i}\left(K_{v}, F\right)$ est bien injective. Le cas $i \leq 2$ avec $\mathcal{F}$ de type multiplicatif résulte de la Prop.4.1. Dans le cas où $\mathcal{F}$ est fini étale avec $i$ quelconque, on a $H^{i}\left(\mathcal{O}_{v}^{h}, \mathcal{F}\right)=H^{i}(\kappa(v), F)$. Soit $G_{v}$ le groupe de Galois de $K_{v}, I_{v}$ le groupe d'inertie et $G(v)$ le groupe de Galois du corps résiduel $\kappa(v)$. L'injectivité voulue résulte alors de ce que la suite exacte de groupes

$$
1 \rightarrow I_{v} \rightarrow G_{v} \rightarrow G(v) \rightarrow 1
$$

est scindée car $k$ est de caractéristique zéro.

Pour démontrer f), on note d'abord qu'il suffit de traiter le cas où $v \notin U$; en effet, si $v \in U$, on choisit un ouvert non vide $V \subset U$ tel que $v \notin V$ et on utilise le diagramme (dont le rectangle supérieur est commutatif)

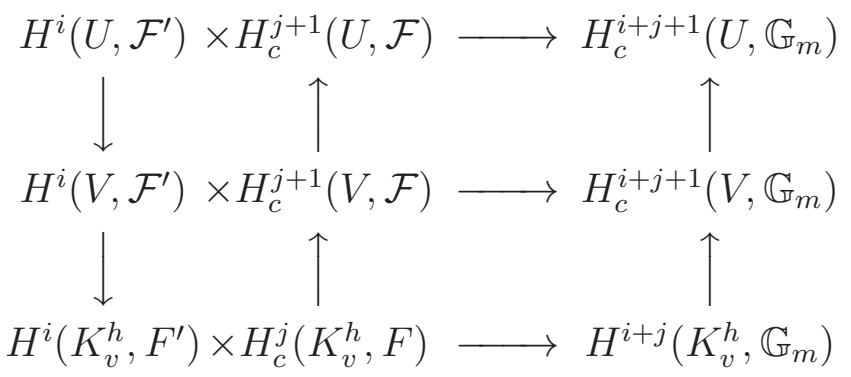

pour se ramener (via c)) à vérifier la commutativité du rectangle inférieur, donc à montrer f) quand $v \notin U$. Comme les accouplements d'Artin-Verdier

$$
H^{i}\left(U, \mathcal{F}^{\prime}\right) \times H_{c}^{j+1}(U, \mathcal{F}) \rightarrow H_{c}^{i+j+1}\left(U, \mathbb{G}_{m}\right)
$$

sont induits par les accouplements

$$
\operatorname{Ext}_{U}^{i}\left(\mathcal{F}, \mathbb{G}_{m}\right) \times H_{c}^{j+1}(U, \mathcal{F}) \rightarrow H_{c}^{i+j+1}\left(U, \mathbb{G}_{m}\right)
$$

et de même pour les accouplements locaux, il suffit de vérifier que le diagramme

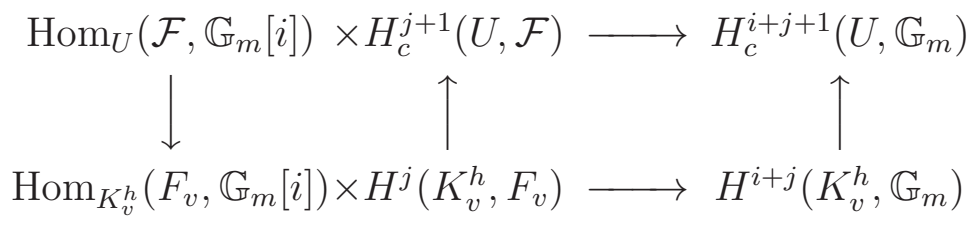


commute, les Hom étant pris dans la catégorie dérivée. Soit alors $\alpha_{U} \in$ $\operatorname{Hom}_{U}\left(\mathcal{F}, \mathbb{G}_{m}[i]\right)$, de restriction $\alpha_{v} \in \operatorname{Hom}_{K_{v}^{h}}\left(F_{v}, \mathbb{G}_{m}[i]\right)$. Comme on l'a vu dans la preuve de $\mathrm{c})$, on a $H^{j}\left(K_{v}^{h}, F\right)=H_{v}^{j+1}\left(\mathcal{O}_{v}^{h},\left(j_{v}\right)_{!} F_{v}\right)$. On conclut avec la commutativité du diagramme

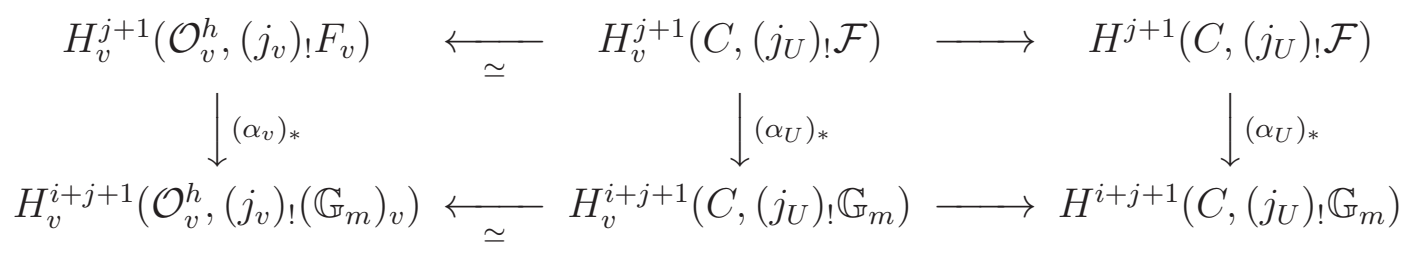

et la définition de la flèche $H^{j}\left(K_{v}^{h}, F\right) \rightarrow H_{c}^{j+1}(U, \mathcal{F})=H^{j+1}\left(C,\left(j_{U}\right) ! \mathcal{F}\right)$, rappelée dans la preuve de c) (les isomorphismes indiqués sur le diagramme sont obtenus via l'excision). La preuve pour $\mathcal{F}$ et $\mathcal{F}^{\prime}$ échangés est identique.

Lemme 4.4. - Soit $k$ un corps à cohomologie galoisienne finie. Soient $C$ une $k$-courbe projective, lisse, géométriquement intègre, et $K=k(C)$. Soit $U \subset C$ un ouvert non vide. Pour tout $U$-schéma en groupes abéliens $\mathcal{F}$ fini étale sur $U$, et tout entier $i \geq 0$, les groupes $H^{i}(U, \mathcal{F})$ et $H_{c}^{i}(U, \mathcal{F})$ sont finis, et ils sont de plus nuls pour $i \geq 4$ si $\mathrm{cd}(k) \leq 1$.

Démonstration. - Pour tout $\mathbb{Z} / n$-faisceau localement constant $\mathcal{F}$ sur toute $k$-variété $U$, les groupes $H^{i}(U, \mathcal{F})$ sont finis, et nuls pour $i$ strictement plus grand que $\operatorname{cd}(k)+2 \operatorname{dim}(U)$ (ceci résulte de la suite spectrale de HochschildSerre et de [23], Th. VI.1.1. et Th. VI.5.5. qui valent pour $k$ algébriquement clos). L'énoncé pour $H_{c}^{i}$ s'en déduit via la suite (4.1) et le fait que les hensélisés $K_{v}^{h}$ sont à cohomologie galoisienne finie, et de plus de dimension cohomologique $\leq 2 \operatorname{sicd}(k) \leq 1$.

Proposition 4.5. - Soit $k$ un corps à cohomologie galoisienne finie. Soient $C$ une $k$-courbe projective, lisse, géométriquement intègre, et $K=k(C)$. Soit $U \subset C$ un ouvert non vide et $\mathcal{T}$ un tore sur $U$.

(i) Pour $i \geq 2$, les groupes $H^{i}(U, \mathcal{T})$ et $H_{c}^{i}(U, \mathcal{T})$ sont de torsion et de type cofini.

(ii) Pour $i \geq 2$, les groupes $H^{i}(U, \widehat{\mathcal{T}})$ et $H_{c}^{i}(U, \widehat{\mathcal{T}})$ sont de torsion et de type cofini.

(iii) Le groupe $H^{1}(U, \widehat{\mathcal{T}})$ est fini et le groupe $H^{0}(U, \widehat{\mathcal{T}})$ est de type fini.

(iv) Les groupes $H^{0}(U, \mathcal{T})_{\text {tors }}$ et $H^{1}(U, \mathcal{T})_{\text {tors }}$ sont de type cofini.

(v) Pour $i \geq 0$, les groupes $H^{i}(U, \mathcal{T}) / n$ et $H^{i}(U, \widehat{\mathcal{T}}) / n$ sont finis. 
Démonstration. - Pour tout schéma régulier $X$, le groupes $H^{i}\left(X, \mathbb{G}_{m}\right)$ sont de torsion pour $i \geq 2$ ([13, II, Prop. 1.4]. Par suite spectrale, on conclut qu'il en est de même pour $H^{i}(X, \mathcal{T})$ pour tout $X$-tore isotrivial. Comme pour tout $n>0$ le groupe $H^{i}\left(U,{ }_{n} \mathcal{T}\right)$ est fini (Lemme 4.4) et se surjecte $\operatorname{sur}_{n} H^{i}(U, \mathcal{T})$ via la suite de Kummer, on obtient aussi que $H^{i}(U, \mathcal{T})$ est de type cofini pour $i \geq 2$. De même $H^{i}(U, \mathcal{T}) / n$ s'injecte dans $H^{i+1}\left(U_{, n} \mathcal{T}\right)$, donc il est aussi fini.

Pour $n>0$ le groupe ${ }_{n} H^{1}(U, \mathcal{T})$ est un quotient du groupe fini $H^{1}(U, n \mathcal{T})$, ce qui montre que $H^{1}(U, \mathcal{T})_{\text {tors }}$ est de type cofini (mais $H^{1}(U, \mathcal{T})$ n'est pas forcément de torsion). Le groupe $H^{0}(U, \mathcal{T})_{\text {tors }}$ est également de type cofini car on a ${ }_{n} H^{0}(U, \mathcal{T})=H^{0}\left(U_{n} T\right)$.

Pour tout schéma normal intègre $X$, et $i \geq 1$, les groupes $H^{i}(X, \mathbb{Z})$ sont de torsion [24], Lemme II.2.10 et le groupe $H^{1}(X, \mathbb{Z})$ est nul par [12], IX.3.6. (ii). Comme le groupe $H^{i-1}(U, \widehat{\mathcal{T}} / n)$ est fini (Lemme 4.4) et se surjecte sur ${ }_{n} H^{i}(U, \widehat{\mathcal{T}})$, on voit que $H^{i}(U, \widehat{\mathcal{T}})$ est encore de type cofini pour tout $i \geq 1$. De plus, comme $H^{1}(U, \mathbb{Z})=0$ pour tout $U$ régulier, il existe (par restrictioncorestriction) un $n>0$ fixé tel que $H^{1}(U, \widehat{\mathcal{T}})={ }_{n} H^{1}(U, \widehat{\mathcal{T}})$, et ce groupe est fini puisque c'est un quotient de $H^{0}(U, \widehat{\mathcal{T}} / n)$. Le groupe $H^{i}(U, \widehat{\mathcal{T}}) / n$ est également fini car il s'injecte dans $H^{i}(U, \widehat{\mathcal{T}} / n)$, et de même $H^{0}(U, \widehat{\mathcal{T}})=$ $H^{0}(K, \widehat{T})$ est de type fini.

De la suite exacte

$$
\bigoplus_{v \notin U} H^{i-1}\left(K_{v}, T\right) \rightarrow H_{c}^{i}(U, \mathcal{T}) \rightarrow H^{i}(U, \mathcal{T})
$$

on déduit que pour $i \geq 2$, le groupe $H_{c}^{i}(U, \mathcal{T})$ est de torsion.

De la suite exacte

$$
\bigoplus_{v \notin U} H^{i-1}\left(K_{v}, \widehat{\mathcal{T}}\right) \rightarrow H_{c}^{i}(U, \widehat{\mathcal{T}}) \rightarrow H^{i}(U, \widehat{\mathcal{T}})
$$

on déduit que pour $i \geq 2$, le groupe $H_{c}^{i}(U,, \widehat{\mathcal{T}})$ est de torsion et de type cofini.

Proposition 4.6. - Soit $C$ une $\mathbb{C}((t))$-courbe projective, lisse, géométriquement intègre. Soit $K=\mathbb{C}((t))(C)$ et $K_{v}$ le complété de $K$ en un point de codimension 1 de $C$.

(i) Pour tout $K$-groupe abélien fini $G$, et tout $i \geq 0$, les groupes

$$
\amalg^{i}(K, G):=\operatorname{Ker}\left[H^{i}(K, G) \rightarrow \prod_{v \in C^{(1)}} H^{i}\left(K_{v}, G\right)\right]
$$


sont finis. Le groupe $\amalg_{\omega}^{1}(K, G)$ est également fini.

(ii) Pour tout $K$-groupe de type multiplicatif $G$, le groupe

$$
\amalg^{1}(K, G):=\operatorname{Ker}\left[H^{1}(K, G) \rightarrow \prod_{v \in C^{(1)}} H^{1}\left(K_{v}, G\right)\right]
$$

est fini.

Démonstration. - Soit $U \subset C$ un ouvert sur lequel le groupe $G$ s'étend en un groupe de type multiplicatif $\mathcal{G}$. D'après la proposition 4.2, les groupes considérés sont dans l'image de $H^{i}(U, \mathcal{G}) \rightarrow H^{i}(K, G)$. Compte tenu du lemme 4.4 ceci établit le premier énoncé de (i). Si maintenant l'action de Galois sur $G(\bar{K})$ est triviale, la proposition 2.6 donne que $\amalg_{\omega}^{1}(K, G)=$ $\amalg^{1}(K, G)$ est fini. Dans le cas général, soit $L$ une extension finie galoisienne de groupe $\Gamma$ de $K$ qui déploie $G$, alors la suite exacte de restriction-inflation induit une suite exacte

$$
0 \rightarrow N \rightarrow \amalg_{\omega}^{1}(K, G) \rightarrow \amalg_{\omega}^{1}(L, G),
$$

où $N$ est un sous-groupe du groupe fini $H^{1}(\Gamma, G)$, d'où la finitude de $\amalg_{\omega}^{1}(K, G)$.

Montrons (ii). Le groupe $H^{1}(K, G)$ est d'exposant fini, disons $n>0$. L'image de $H^{1}(U, \mathcal{G}) \rightarrow H^{1}(K, G)$ se factorise donc par $H^{1}(U, \mathcal{G}) / n$. On a une suite exacte de $U$-groupes de type multiplicatif

$$
1 \rightarrow \mathcal{T} \rightarrow \mathcal{G} \rightarrow \mathcal{F} \rightarrow 1
$$

où $\mathcal{T}$ est un $U$-tore et $\mathcal{F}$ un $U$-groupe abélien fini. Soit $m$ un multiple de $n$ qui annule $F$. On a alors une suite exacte

$$
H^{1}(U, \mathcal{T}) / m \rightarrow H^{1}(U, \mathcal{G}) / n \rightarrow H^{1}(U, \mathcal{F})
$$

et une injection

$$
H^{1}(U, \mathcal{T}) / m \hookrightarrow H^{2}\left(U,_{m} \mathcal{T}\right) .
$$

Comme $H^{1}(U, \mathcal{F})$ et $H^{2}(U, m \mathcal{T})$ sont finis (Lemme 4.4), ceci établit (ii).

Remarque 4.7. - À la différence du cas classique, celui des corps de nombres, et du cas des corps de fonctions d'une variable sur un corps $p$ adique [17]), sur $K=\mathbb{C}((t))(C)$, le groupe $\amalg^{2}\left(K, \mathbb{G}_{m}\right)$ n'est pas forcément fini. Si la courbe $C$ admet un modèle projectif et lisse sur $\mathbb{C}[[t]]$ de fibre spéciale $C_{0}$ de genre $g$, alors $\amalg^{2}\left(K, \mathbb{G}_{m}\right) \simeq H^{1}\left(C_{0}, \mathbb{Q} / \mathbb{Z}\right) \simeq(\mathbb{Q} / \mathbb{Z})^{2 g}($ voir la proposition 2.97. 


\section{Dualité à la Artin-Verdier sur un ouvert}

Proposition 5.1. - Pour un U-schéma en groupes abéliens finis étale $\mathcal{F}$, de dual de Cartier $\mathcal{F}^{0}=\operatorname{Hom}(\mathcal{F}, \mathbb{Q} / \mathbb{Z}(1))$, on a, pour $0 \leq i \leq 3$, un accouplement parfait de groupes finis

$$
H^{i}(U, \mathcal{F}) \times H_{c}^{3-i}\left(U, \mathcal{F}^{0}\right) \rightarrow H_{c}^{3}(U, \mathbb{Q} / \mathbb{Z}(1))=\mathbb{Q} / \mathbb{Z}(-1) .
$$

Démonstration. - L'argument est ici exactement le même que dans [[23], Corollaire V.2.3. Il s'agit de combiner la dualité de Poincaré pour une variété projective et lisse sur un corps algébriquement clos avec la dualité en cohomologie galoisienne sur le corps $\mathbb{C}((t))$. Pour un argument détaillé dans un cadre général, voir [22].

Pour conserver la symétrie, il est commode ici d'introduire les 1-motifs $\mathcal{M}$ sur $U$ de la forme $\mathcal{M}=\mathcal{T}=[0 \rightarrow \mathcal{T}]$, dont le dual est le 1-motif $\mathcal{M}^{*}=\widehat{\mathcal{T}}[1]=[\widehat{\mathcal{T}} \rightarrow 0]$ (par convention, dans ces complexes à deux termes, le terme de gauche est placé en degré -1 et celui de droite en degré 0$)$. On a alors une flèche (dans la catégorie dérivée bornée des faisceaux fppf sur $U$ ) :

$$
\mathcal{M} \otimes{ }^{\mathfrak{L}} \mathcal{M}^{*} \rightarrow \mathbb{G}_{m}[1]
$$

qui induit pour $0 \leq i \leq 2$ des accouplements

$$
H^{i}(U, \mathcal{M}) \times H_{c}^{2-i}\left(U, \mathcal{M}^{*}\right) \rightarrow H_{c}^{3}\left(U, \mathbb{G}_{m}\right)=\mathbb{Q} / \mathbb{Z}(-1) .
$$

(Noter qu' on peut ici utiliser indifféremment la cohomologie fppf ou étale, les schémas en groupes qui interviennent étant supposés lisses). On dispose aussi pour tout $n>0$ de la réalisation $n$-adique $T_{\mathbb{Z} / n} \mathcal{M}$ de $\mathcal{M}$, qui est un schéma en groupes fini étale sur $U$, et d'un triangle exact associé

$$
T_{\mathbb{Z} / n} \mathcal{M} \rightarrow \mathcal{M} \rightarrow \mathcal{M} \rightarrow T_{\mathbb{Z} / n} \mathcal{M}[1] .
$$

Le dual de Cartier de $T_{\mathbb{Z} / n} \mathcal{M}$ est $\left(T_{\mathbb{Z} / n} \mathcal{M}\right)^{0}=T_{\mathbb{Z} / n} \mathcal{M}^{*}$. Si $M=\mathcal{T}$, on a simplement $T_{\mathbb{Z} / n} \mathcal{M}={ }_{n} \mathcal{T}$, tandis que si $M=\widehat{\mathcal{T}}[1]$, on a $T_{\mathbb{Z} / n} \mathcal{M}=\widehat{\mathcal{T}} / n$. La flèche

$$
T_{\mathbb{Z} / n} \mathcal{M} \otimes T_{\mathbb{Z} / n} \mathcal{M}^{*} \rightarrow \mu_{n}
$$

associée à cette dualité est compatible (via le lemme 3.1) avec (5.1), i.e. le diagramme

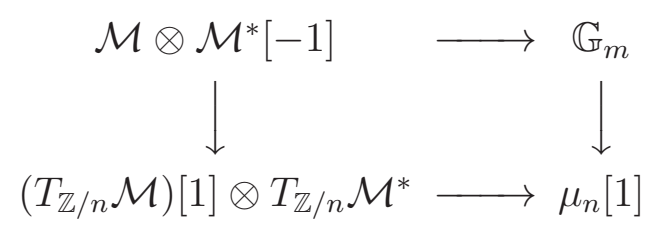


est commutatif.

Théorème 5.2. - Soit $\mathcal{M}$ un 1-motif sur $U$ du type $\mathcal{M}=\mathcal{T}$ ou $\mathcal{M}=\widehat{\mathcal{T}}[1]$, où $\mathcal{T}$ est un tore. Soit $\mathcal{M}^{*}$ le dual de $\mathcal{M}$. Soit l un nombre premier. Alors pour $0 \leq i \leq 2$, l'accouplement

$$
H^{i}(U, \mathcal{M}) \times H_{c}^{2-i}\left(U, \mathcal{M}^{*}\right) \rightarrow \mathbb{Q} / \mathbb{Z}(-1)
$$

induit des accouplements parfaits de groupes finis

$$
H^{i}(U, \mathcal{M})\{l\}^{(l)} \times H_{c}^{2-i}\left(U, \mathcal{M}^{*}\right)^{(l)}\{l\} \rightarrow \mathbb{Q}_{l} / \mathbb{Z}_{l}(-1)
$$

et

$$
H_{c}^{i}(U, \mathcal{M})\{l\}^{(l)} \times H^{2-i}\left(U, \mathcal{M}^{*}\right)^{(l)}\{l\} \rightarrow \mathbb{Q}_{l} / \mathbb{Z}_{l}(-1)
$$

Démonstration. - C'est essentiellement le même argument que dans [17] (Th. 1.3) ou [15] (Th. 3.4). Le triangle exact (5.2) induit pour tout $n>0$ un diagramme commutatif (cf. (5.3) ) de groupes finis (rappelons que $T_{\mathbb{Z} / n} \mathcal{M}$ est un schéma en groupes fini étale sur $U$ ), à lignes exactes :

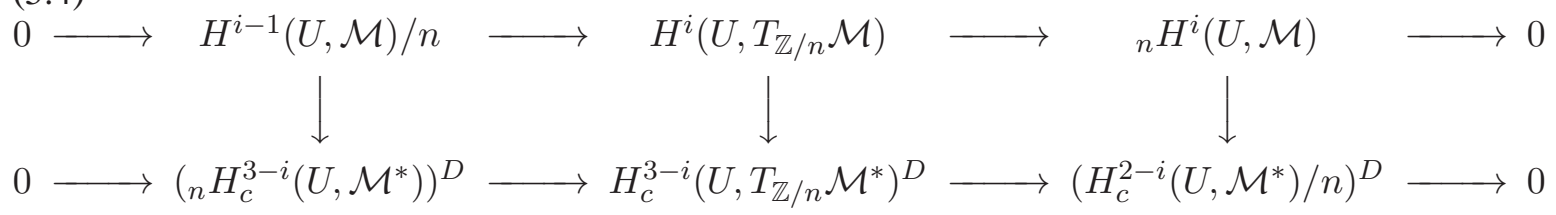

La flèche verticale du milieu est un isomorphisme par la Prop. 5.1. En prenant $n=l^{m}$ (pour $m=1,2, \ldots$ ) et en passant à la limite inductive sur $m$, on obtient un diagramme commutatif à lignes exactes et dont la flèche verticale du milieu est un isomorphisme :

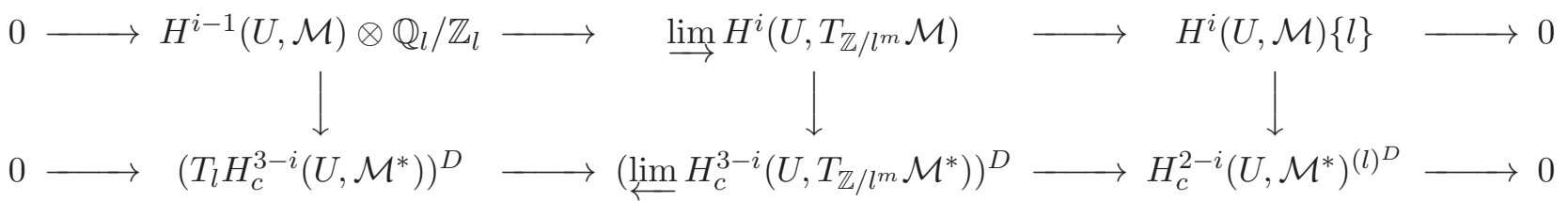

Maintenant comme $H^{i-1}(U, \mathcal{M}) \otimes \mathbb{Q}_{l} / \mathbb{Z}_{l}$ est divisible, ce diagramme induit un isomorphisme

$$
\left(\lim _{\longrightarrow} H^{i}\left(U, T_{\mathbb{Z} / l^{m}} \mathcal{M}\right)\right)^{(l)} \simeq\left(H^{i}(U, \mathcal{M})\{l\}\right)^{(l)} .
$$

Comme le module de Tate $T_{l} H_{c}^{3-i}\left(U, \mathcal{M}^{*}\right)$ est sans torsion, on a de même un isomorphisme

$$
H_{c}^{2-i}\left(U, \mathcal{M}^{*}\right)^{(l)}\{l\} \simeq \lim _{\longleftarrow} H_{c}^{3-i}\left(U, T_{\mathbb{Z} / l^{m}} \mathcal{M}^{*}\right)\{l\}
$$

qui induit par dualité un isomorphisme

$$
\left(\lim _{\longleftarrow} H_{c}^{3-i}\left(U, T_{\mathbb{Z} / l^{m}} \mathcal{M}^{*}\right)\right)\{l\}^{D} \simeq H_{c}^{2-i}\left(U, \mathcal{M}^{*}\right)^{(l)}\{l\}^{D},
$$


ce qui démontre finalement que la flèche verticale de droite du diagramme précédent induit un isomorphisme entre les groupes $H^{i}(U, \mathcal{M})\{l\}^{(l)}$ et $H_{c}^{2-i}\left(U, \mathcal{M}^{*}\right)^{(l)}\{l\}^{D}$. On démontre de même qu'on a un isomorphisme entre $\left.H_{c}^{i}(U, \mathcal{M})\{l\}\right)^{(l)}$ et $H^{2-i}\left(U, \mathcal{M}^{*}\right)^{(l)}\{l\}^{D}$ en remplaçant les groupes $H^{i}$ par les groupes $H_{c}^{i}$ et vice-versa dans le diagramme 5.4 .

En prenant successivement $\mathcal{M}=\mathcal{T}$ et $\mathcal{M}=\widehat{\mathcal{T}}[1]$, on obtient :

Corollaire 5.3. - Soit $i$ un entier avec $0 \leq i \leq 3$.

(i) L'accouplement

$$
H^{i}(U, \mathcal{T}) \times H_{c}^{3-i}(U, \widehat{\mathcal{T}}) \rightarrow \mathbb{Q} / \mathbb{Z}(-1) .
$$

induit des accouplements parfaits de groupes finis

$$
H^{i}(U, \mathcal{T})\{l\}^{(l)} \times H_{c}^{3-i}(U, \widehat{\mathcal{T}})^{(l)}\{l\} \rightarrow \mathbb{Q}_{l} / \mathbb{Z}_{l}(-1)
$$

et

$$
H^{i}(U, \mathcal{T})^{(l)}\{l\} \times H_{c}^{3-i}(U, \widehat{\mathcal{T}})\{l\}^{(l)} \rightarrow \mathbb{Q}_{l} / \mathbb{Z}_{l}(-1)
$$

(ii) L'accouplement

$$
H^{i}(U, \widehat{\mathcal{T}}) \times H_{c}^{3-i}(U, \mathcal{T}) \rightarrow \mathbb{Q} / \mathbb{Z}(-1) .
$$

induit des accouplements parfaits de groupes finis

$$
H^{i}(U, \widehat{\mathcal{T}})\{l\}^{(l)} \times H_{c}^{3-i}(U, \mathcal{T})^{(l)}\{l\} \rightarrow \mathbb{Q}_{l} / \mathbb{Z}_{l}(-1)
$$

et

$$
H^{i}(U, \widehat{\mathcal{T}})^{(l)}\{l\} \times H_{c}^{3-i}(U, \mathcal{T})\{l\}^{(l)} \rightarrow \mathbb{Q}_{l} / \mathbb{Z}_{l}(-1)
$$

Remarque 5.4. - Les énoncés de ce paragraphe sont valables pour un 1motif quelconque (y compris comportant une variété abélienne) ; la preuve est identique via le fait (bien connu, mais pour lequel il est difficile de trouver une référence) qu'on a encore le diagramme commutatif (5.3) dans ce contexte. Par contre les résultats du paragraphe suivant ne sont pas valables pour des 1-motifs quelconques, faute d'avoir un bon théorème de dualité locale pour les variétés abéliennes sur les complétés $K_{v}$ de $K$. 


\section{Groupes abéliens finis : dualité pour les groupes de Tate-Shafarevich}

Dans toute cette section, on pose $k=\mathbb{C}((t))$, et on désigne par $C$ une $k$-courbe projective, lisse, géométriquement intègre, de corps des fonctions $K=k(C)$. Soit $\mathcal{F}$ un groupe de type multiplicatif sur $U$ de dual de Cartier $\mathcal{F}^{\prime}=\operatorname{Hom}\left(\mathcal{F}, \mathbb{G}_{m}\right)$. Notons $F$ et $F^{\prime}$ les fibres génériques respectives sur Spec $K$ de $\mathcal{F}$ et $\mathcal{F}^{\prime}$. Soit $U$ un ouvert non vide de $C$. Pour tout entier $i$ avec $0 \leq i \leq 3$, on a un accouplement d'Artin-Verdier

$$
H^{i}(U, \mathcal{F}) \times H_{c}^{3-i}\left(U, \mathcal{F}^{\prime}\right) \rightarrow H_{c}^{3}\left(U, \mathbb{G}_{m}\right) \stackrel{\sim}{\rightarrow} \mathbb{Q} / \mathbb{Z}(-1)
$$

et pour toute place $v \in C^{(1)}$ un accouplement local

$$
H^{i}\left(K_{v}, F\right) \times H^{2-i}\left(K_{v}, F^{\prime}\right) \rightarrow \operatorname{Br} K_{v} \stackrel{\simeq}{\rightarrow} H_{c}^{3}\left(U, \mathbb{G}_{m}\right) \stackrel{\simeq}{\rightarrow} \mathbb{Q} / \mathbb{Z}(-1) .
$$

De même en échangeant $\mathcal{F}$ et $\mathcal{F}^{\prime}$.

Lemme 6.1. - Avec les notations ci-dessus, on considère les flèches

$$
H^{i}(U, \mathcal{F}) \rightarrow H_{c}^{3-i}\left(U, \mathcal{F}^{\prime}\right)^{D} ; \quad \prod_{v \in C^{(1)}} H^{i}\left(K_{v}, F\right) \rightarrow \bigoplus_{v \in C^{(1)}} H^{2-i}\left(K_{v}, F^{\prime}\right)^{D}
$$

induites respectivement par l'accouplement d'Artin-Verdier et la somme des accouplements locaux. Alors on a un diagramme commutatif

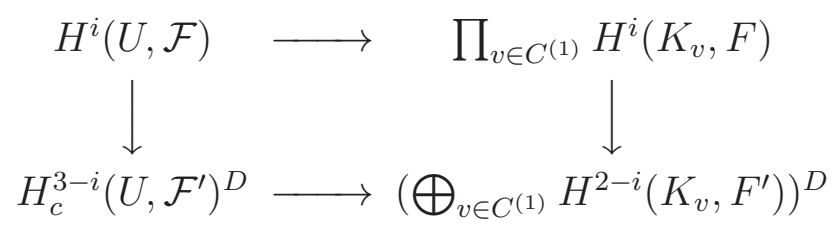

et de même en échangeant $\mathcal{F}$ et $\mathcal{F}^{\prime}$.

Démonstration. - Il suffit d'appliquer la proposition4.3f) avec $j=2-i$ à chaque place $v \in C^{(1)}$, en notant aussi que la compatibilité avec les complétés $K_{v}$ résulte immédiatement de celle avec les hensélisés $K_{v}^{h}$.

Théorème 6.2. - Soit $M$ un $K$-module fini; soit $M^{0}=\operatorname{Hom}_{\mathbb{Z}}(M, \mathbb{Q} / \mathbb{Z}(1))$. On a un accouplement parfait de groupes abéliens finis

$$
\amalg^{1}(K, M) \times \amalg^{2}\left(K, M^{0}\right) \rightarrow \mathbb{Q} / \mathbb{Z}(-1) .
$$

Démonstration. - Le reste de la section est consacré à la démonstration de ce théorème, qui utilise la Prop. 4.3 et aussi les idées de [17], théorème 4.4.

Soit $M$ un $K$-module fini. Soit $U \subset C$ un ouvert non vide sur lequel $M$ (ainsi que $M^{0}$ ) s'étend en un $U$-schéma en groupes abéliens fini étale, encore noté $M$. 
Comme dans [23, II, $\S 2$, p. 178] et [17, §3] (cf. aussi la Prop. 4.3, d), on introduit pour tout entier $i \geq 0$ les groupes

$$
\begin{gathered}
\mathcal{D}^{i}(U, M)=\operatorname{Im}\left[H_{c}^{i}(U, M) \rightarrow H^{i}(K, M)\right] \\
D^{i}(U, M)=\operatorname{Ker}\left[H^{i}(U, M) \rightarrow \prod_{v \notin U} H^{i}\left(K_{v}, M\right)\right]
\end{gathered}
$$

Comme $H_{c}^{i}(U, M)$ et $H^{i}(U, M)$ sont finis, les groupes $\mathcal{D}^{i}(U, M)$ et $D^{i}(U, M)$ sont finis.

Lemme 6.3. - Soient $k=\mathbb{C}((t)), U / k$ une courbe lisse et $M$ un $U$-groupe fini étale. Pour tout $v \in U^{(1)}$, on a $H^{2}\left(\mathcal{O}_{v}, M\right)=0$, et de même avec $\mathcal{O}_{v}^{h}$ au lieu de $\mathcal{O}_{v}$.

Démonstration. - On a $H^{2}\left(\mathcal{O}_{v}, M\right)=H^{2}\left(\kappa_{v}, M_{\kappa_{v}}\right)$, et ce dernier groupe est nul car $\kappa_{v}$ est de dimension cohomologique 1. De même pour $\mathcal{O}_{v}^{h}$.

On voit donc que dans la situation ci-dessus on a

$$
D^{2}(U, M)=\operatorname{Ker}\left[H^{2}(U, M) \rightarrow \prod_{v \in C^{(1)}} H^{2}\left(K_{v}, M\right)\right] .
$$

Par ailleurs la proposition 4.3 (e) s'applique avec $i=1$, et fournit une suite exacte

$$
\bigoplus_{v \in C^{(1)}} H^{0}\left(K_{v}, M^{0}\right) \rightarrow H_{c}^{1}\left(U, M^{0}\right) \rightarrow \mathcal{D}^{1}\left(U, M^{0}\right) \rightarrow 0 .
$$

Comme les groupes $\mathcal{D}^{1}(U, M) \subset H^{1}(K, M)$ sont finis et décroissent avec $U$ (Prop.4.3 d), ceci implique qu'il existe un ouvert non vide $U_{0} \subset U$ tel que pour tout ouvert non vide $V \subset U_{0}$ de $C$, on ait

$$
\mathcal{D}^{1}\left(V, M^{0}\right)=\mathcal{D}^{1}\left(U_{0}, M^{0}\right) .
$$

On a alors aussi $\mathcal{D}^{1}\left(U_{0}, M^{0}\right)=\amalg^{1}\left(K, M^{0}\right)$. En effet si $\alpha$ est un élément de $\mathcal{D}^{1}\left(U_{0}, M^{0}\right)$, alors il est pour tout $V \subset U_{0}$ dans $\mathcal{D}^{1}\left(V, M^{0}\right)$, donc par définition dans l'image de $H_{c}^{1}\left(V, M^{0}\right)$, ce qui implique que sa restriction $\alpha_{v}$ à $H^{1}\left(K_{v}^{h}, M^{0}\right)$ (et donc aussi à $H^{1}\left(K_{v}, M^{0}\right)$ ) est nulle pour tout $v \notin V$ via la suite exacte (4.1). Ceci étant vrai pour tout ouvert non vide $V \subset U_{0}$, on obtient que $\mathcal{D}^{1}\left(U_{0}, M^{0}\right) \subset \amalg^{1}\left(K, M^{0}\right)$. En sens inverse, tout élément de $\amalg^{1}\left(K, M^{0}\right)$ se relève dans $H^{1}\left(U_{0}, M^{0}\right)$ (Prop. 4.2, ii), en un élément qui provient de $H_{c}^{1}\left(U_{0}, M^{0}\right)$ d'après la suite exacte (4.1), ce qui prouve l'inclusion en sens inverse.

Quitte à rétrécir $U$, on peut supposer que $U=U_{0}$. 
On considère alors le diagramme (commutatif d'après le lemme 6.1) suivant de suites exactes

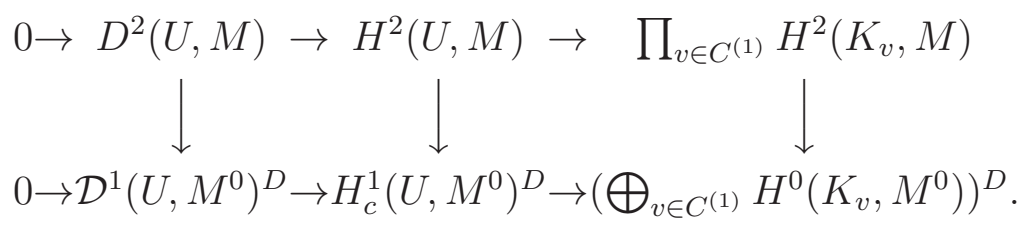

D'après la proposition 5.1, la flèche médiane est un isomorphisme. Les théorèmes de dualité locale (Prop. 3.3) donnent que la flèche de droite est un isomorphisme.

La flèche induite

$$
D^{2}(U, M) \rightarrow \mathcal{D}^{1}\left(U, M^{0}\right)^{D}
$$

est donc un isomorphisme de groupes abéliens finis. On a $\mathcal{D}^{1}\left(U, M^{0}\right)=$ $\amalg^{1}\left(K, M^{0}\right)$. Il reste à voir le lien entre $D^{2}(U, M)$ et $\amalg^{2}(K, M)$. En utilisant la proposition 4.2 on voit que pour $V \subset U$ avec $M$ un $U$-schéma en groupes finis étales, la flèche de restriction $H^{2}(U, M) \rightarrow H^{2}(V, M)$ induit une surjection de groupes finis $D^{2}(U, M) \rightarrow D^{2}(V, M)$. En considérant les images dans $H^{2}(K, M)$, on voit qu'il existe un ouvert fixe $U_{1} \subset C$ tel que pour tout ouvert non vide $U$ de $C$ les flèches

$$
D^{2}\left(U_{1}, M\right) \rightarrow D^{2}\left(U \cap U_{1}, M\right) \rightarrow \amalg^{2}(K, M)
$$

soient des isomorphismes. Quitte à restreindre encore $U$, on peut donc supposer que $D^{2}(U, M)=\amalg^{2}(K, M)$, ce qui termine la preuve.

\section{Tores : dualités pour les groupes de Tate-Shafarevich}

Soit $U \subset C$ un ouvert non vide et $\mathcal{T}$ un tore sur $U$ de fibre générique $T$. Pour tout groupe abélien $A$, on note $\bar{A}$ le quotient de $A$ par son sous-groupe divisible maximal.

Théorème 7.1. - On a un accouplement parfait de groupes finis

$$
\amalg^{1}(K, \widehat{T}) \times \overline{\amalg^{2}(K, T)} \rightarrow \mathbb{Q} / \mathbb{Z}(-1) .
$$

Théorème 7.2. - On a un accouplement parfait de groupes finis

$$
\amalg^{1}(K, T) \times \overline{\amalg^{2}(K, \widehat{T})} \rightarrow \mathbb{Q} / \mathbb{Z}(-1) .
$$


Démonstration. - On suit la méthode de [17], théorème 4.1, en commençant par des observations qui serviront pour les deux énoncés. Pour $i=1,2$, et $\mathcal{T}$ tore $\operatorname{sur} U$, on va encore utiliser les groupes

$$
\mathcal{D}^{i}(U, \mathcal{T})=\operatorname{Im}\left[H_{c}^{i}(U, \mathcal{T}) \rightarrow H^{i}(K, T)\right] .
$$

Pour $i=1$, c'est un groupe fini car $H^{1}(K, T)$ est d'exposant fini et l'image se factorise donc par un quotient $H_{c}^{1}(U, \mathcal{T}) / n \subset H_{c}^{2}\left(U,{ }_{n} \mathcal{T}\right)$. Les groupes $H_{c}^{2}(U, \mathcal{T})$ et $H^{2}(U, T)$ sont de torsion, de type cofini. Ceci implique que les groupes $\mathcal{D}^{2}(U, \mathcal{T})\{l\}$ sont de type cofini. Fixons un nombre premier $l$. Comme dans la prop. 3.6 de [17] il existe un ouvert $U_{0}$ (dépendant de $l$ ) tel que pour tout ouvert non vide $V \subset U_{0}$ on ait $\mathcal{D}^{i}(V, \mathcal{T})\{l\}=\mathcal{D}^{i}(V, \mathcal{T})\{l\} \subset$ $H^{i}(K, T)$ pour $i=1,2$. Quitte à restreindre $U$, on peut supposer $U_{0}=U$. Comme dans la Prop. 3.6 de [17], on a alors

$$
\mathcal{D}^{i}(U, T)\{l\}=\amalg^{i}(K, T)\{l\}
$$

(et de même pour tout ouvert non vide inclus dans $U$ ), puisque si $V \subset U$ tout élément de $H_{c}^{i}(V, \mathcal{F})$ a une image nulle dans $H^{i}\left(K_{v}, F\right)$ pour $v \notin V$.

On va aussi utiliser

$$
\mathcal{D}^{i}(U, \widehat{\mathcal{T}})=\operatorname{Im}\left[H_{c}^{i}(U, \widehat{\mathcal{T}}) \rightarrow H^{i}(K, \widehat{T})\right] .
$$

Pour $i=1 \mathrm{c}$ 'est un groupe fini. Pour $i \geq 2 \mathrm{c}$ 'est un groupe de torsion de type cofini. Là encore on peut supposer que pour tout ouvert non vide $V \subset U$ on a $\mathcal{D}^{i}(V, \widehat{\mathcal{T}})\{l\}=\mathcal{D}^{i}(U, \widehat{\mathcal{T}})\{l\} \subset H^{i}(K, \widehat{T})$ pour $i=1,2$.

Partant de là on obtient comme ci-dessus

$$
\left.\mathcal{D}^{i}(U, \widehat{T})\right)\{l\}=\amalg^{i}(K, \widehat{T})\{l\},
$$

et de même pour tout ouvert non vide inclus dans $U$. Pour $i=2$, ce groupe n'est pas forcément fini.

\section{Preuve du théorème 7.1}

La prop.4.3 e) s'applique à $\mathcal{T}$ pour $i=2$ (noter qu'ici $H^{2}\left(\mathcal{O}_{v}, \mathcal{T}\right)=0$ car le corps résiduel $\kappa(v)$ de $\mathcal{O}_{v}$ est de dimension cohomologique 1 ), et donne la suite exacte de groupes de torsion

$$
\bigoplus_{v \in C^{(1)}} H^{1}\left(K_{v}, T\right) \rightarrow H_{c}^{2}(U, \mathcal{T}) \rightarrow \mathcal{D}^{2}(U, \mathcal{T}) \rightarrow 0
$$

On a des suites exactes induites

$$
\bigoplus_{v \in C^{(1)}} H^{1}\left(K_{v}, T\right)\{l\} \rightarrow H_{c}^{2}(U, \mathcal{T})\{l\} \rightarrow \mathcal{D}^{2}(U, \mathcal{T})\{l\} \rightarrow 0 .
$$


On a des suites exactes

$$
0 \rightarrow H_{c}^{2}(U, \mathcal{T})\{l\}_{\text {Div }} \rightarrow H_{c}^{2}(U, \mathcal{T})\{l\} \rightarrow \overline{H_{c}^{2}(U, \mathcal{T})\{l\}} \rightarrow 0
$$

et

$$
0 \rightarrow \mathcal{D}^{2}(U, \mathcal{T})\{l\}_{\text {Div }} \rightarrow \mathcal{D}^{2}(U, \mathcal{T})\{l\} \rightarrow \overline{\mathcal{D}^{2}(U, \mathcal{T})\{l\}} \rightarrow 0 .
$$

En prenant les duaux $A \mapsto A^{D}$ (à coefficients $\mathbb{Q}_{l} / \mathbb{Z}_{l}(-1)$ ), on obtient deux suites exactes

$$
0 \rightarrow{\overline{H_{c}^{2}(U, \mathcal{T})\{l\}}}^{D} \rightarrow H_{c}^{2}(U, \mathcal{T})\{l\}^{D} \rightarrow\left[H_{c}^{2}(U, \mathcal{T})\{l\}_{\text {Div }}\right]^{D} \rightarrow 0
$$

et

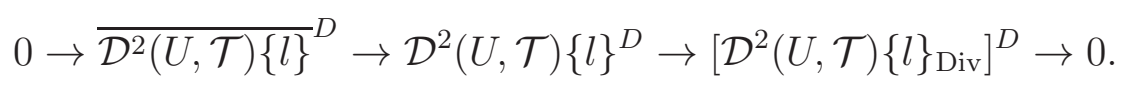

Dans ces deux dernières suites, les groupes de gauche sont finis, les groupes de droite sont des $\mathbb{Z}_{l}$-modules de type fini sans torsion.

On définit

$$
D_{s h}^{1}(U, \widehat{\mathcal{T}})=\operatorname{Ker}\left[H^{1}(U, \widehat{\mathcal{T}}) \rightarrow \prod_{v \in C^{(1)}} H^{1}\left(K_{v}, \widehat{T}\right)\right] .
$$

Le groupe $H^{1}(U, \widehat{\mathcal{T}})$ est fini, donc aussi $D_{\text {sh }}^{1}(U, \widehat{\mathcal{T}})$.

On a le diagramme (commutatif par le lemme 6.1)

$$
\begin{aligned}
& 0 \quad \rightarrow \quad D_{s h}^{1}(U, \widehat{\mathcal{T}})\{l\} \quad \rightarrow \quad H^{1}(U, \widehat{\mathcal{T}})\{l\} \quad \rightarrow \quad \prod_{v \in C^{(1)}} H^{1}\left(K_{v}, \widehat{T}\right)\{l\} \\
& 0 \rightarrow \mathcal{D}^{2}(U, \stackrel{\downarrow}{\mathcal{T}})\{l\}^{D} \rightarrow H_{c}^{2}(U, \stackrel{\downarrow}{\mathcal{T}})\{l\}^{D} \rightarrow\left[\bigoplus_{v \in C^{(1)}} H^{1}\left(K_{v}, T\right)\{l\}\right]^{D}
\end{aligned}
$$

Dans ce diagramme, les groupes $D_{s h}^{1}(U, \widehat{\mathcal{T}})$ et $H^{1}(U, \widehat{\mathcal{T}})$ sont finis.

Il résulte alors de ce qui précède, et du fait que les groupes de droite sont d'exposant fini, que ce diagramme se factorise en un diagramme commutatif de suites exactes :

$$
\begin{aligned}
& 0 \rightarrow D_{s h}^{1}(U, \widehat{\mathcal{T}})\{l\} \quad \rightarrow \quad H^{1}(U, \widehat{\mathcal{T}})\{l\} \quad \rightarrow \quad \prod_{v \in C^{(1)}} H^{1}\left(K_{v}, \widehat{T}\right)\{l\} \\
& 0 \rightarrow{\frac{\downarrow}{\mathcal{D}^{2}(U, \mathcal{T})\{l\}}}^{D} \rightarrow{\frac{\downarrow}{H_{c}^{2}(U, \mathcal{T})\{l\}}}^{D} \rightarrow\left[\bigoplus_{v \in C^{(1)}} H^{1}\left(K_{v}, T\right)\{l\}\right]^{D} .
\end{aligned}
$$

On a vu que l'on a des accouplements parfaits de groupes finis

$$
H^{i}(U, \widehat{\mathcal{T}})^{(l)}\{l\} \times H_{c}^{3-i}(U, \mathcal{T})\{l\}^{(l)} \rightarrow \mathbb{Q}_{l} / \mathbb{Z}_{l}(-1) .
$$

Appliquant ceci à $i=1$ et utilisant le fait que $H^{1}(U, \widehat{T)}$ est fini, on voit que la flèche verticale médiane est un isomorphisme. Par les dualités locales, la 
flèche verticale de droite est un isomorphisme. On obtient donc une dualité parfaite de groupes finis :

$$
D_{\text {sh }}^{1}(U, \widehat{\mathcal{T}})\{l\} \times \overline{\mathcal{D}^{2}(U, \mathcal{T})\{l\}} \rightarrow \mathbb{Q}_{l} / \mathbb{Z}_{l}(-1) .
$$

Or on a déjà vu qu'on avait

$$
\mathcal{D}^{2}(U, \mathcal{T})\{l\}=\amalg^{2}(K, T)\{l\} .
$$

Pour $\amalg^{1}(K, \widehat{T})$, on utilise le lemme suivant.

Lemme 7.3. - Pour tout ouvert $V \subset U$, la flèche de restriction $H^{1}(U, \widehat{\mathcal{T}}) \rightarrow$ $H^{1}(V, \widehat{\mathcal{T}})$ est un isomorphisme, et il en est de même de la restriction $H^{1}(U, \widehat{\mathcal{T}}) \rightarrow H^{1}(K, \widehat{T})$. On a donc $\amalg^{1}(K, \widehat{T})=D_{s h}^{1}(U, \widehat{\mathcal{T}})$.

Démonstration. - Soit $U^{\prime} \rightarrow U$ un revêtement fini étale galoisien intègre, de groupe $G$, déployant $\widehat{\mathcal{T}}$. On a alors la suite exacte

$$
0 \rightarrow H^{1}\left(G, \widehat{\mathcal{T}}\left(U^{\prime}\right)\right) \rightarrow H^{1}(U, \widehat{\mathcal{T}}) \rightarrow H^{1}\left(U^{\prime}, \widehat{\mathcal{T}}\right)
$$

qui, comme $H^{1}\left(U^{\prime}, \mathbb{Z}\right)=0$ (car $U^{\prime}$ est lisse et en particulier normal) donne un isomorphisme $H^{1}\left(G, \widehat{\mathcal{T}}\left(U^{\prime}\right)\right) \simeq H^{1}(U, \widehat{\mathcal{T}})$.

On conclut finalement qu'on a pour chaque nombre premier $l$ une dualité parfaite entre les groupes finis $\overline{\amalg^{2}(K, T)\{l\}}$ et $\amalg^{1}(K, \widehat{T})\{l\}$, ce qui termine la preuve du théorème 7.1 en raisonnant composante $l$-primaire par composante $l$-primaire.

\section{Preuve du théorème 7.2}

On peut appliquer la Prop. 4.3 e) avec $i=1$ à $\widehat{\mathcal{T}}$, car pour $v \in U$ on a un isomorphisme $H^{1}\left(\mathcal{O}_{v}, \widehat{\mathcal{T}}\right) \rightarrow H^{1}\left(K_{v}, \widehat{T}\right)$ via la suite de restriction-inflation et le fait que le groupe d'inertie de $K_{v}$ agit trivialement sur $\widehat{T}$. On en déduit une suite exacte de groupes de torsion

$$
\bigoplus_{v \in C^{(1)}} H^{1}\left(K_{v}, \widehat{T}\right) \rightarrow H_{c}^{2}(U, \widehat{\mathcal{T}}) \rightarrow \mathcal{D}^{2}(U, \widehat{\mathcal{T}}) \rightarrow 0
$$

On définit

$$
D_{s h}^{1}(U, \mathcal{T})=\operatorname{Ker}\left[H^{1}(U, \mathcal{T}) \rightarrow \prod_{v \in C^{(1)}} H^{1}\left(K_{v}, T\right)\right] .
$$

(rappel : ceci n'est en général pas un groupe de torsion.)

On a le diagramme commutatif 


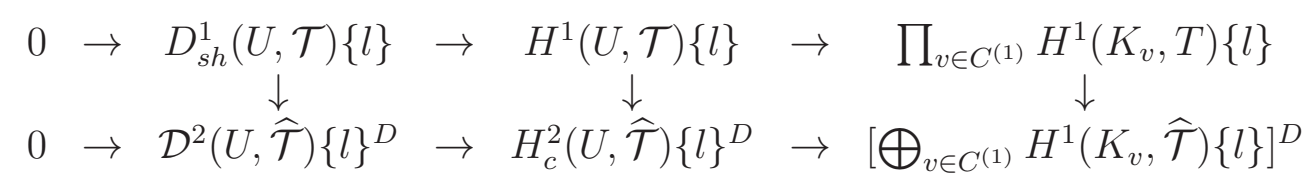

Le théorème donne un accouplement parfait de groupes finis

$$
H^{1}(U, \mathcal{T})\{l\}^{(l)} \times H_{c}^{2}(U, \widehat{\mathcal{T}})^{(l)}\{l\} \rightarrow \mathbb{Q}_{l} / \mathbb{Z}_{l}(-1) .
$$

Compte tenu du fait que $H_{c}^{2}(U, \widehat{\mathcal{T}})$ est un groupe de torsion de type cofini, ceci se lit aussi comme

$$
\overline{H^{1}(U, \mathcal{T})\{l\}} \times \overline{H_{c}^{2}(U, \hat{\mathcal{T}})\{l\}} \rightarrow \mathbb{Q}_{l} / \mathbb{Z}_{l}(-1) .
$$

Comme tout accouplement entre groupes de torsion passe au quotient par les sous-groupes divisibles maximaux, et comme les groupes de droite dans le diagramme sont annulés par un entier fixe, ce diagramme induit un diagramme commutatif de suites exactes

$$
\begin{aligned}
& \begin{array}{rlccc}
0 \rightarrow & \overline{D_{s h}^{1}(U, \mathcal{T})\{l\}} \\
\downarrow & \rightarrow & \overline{H^{1}(U, \mathcal{T})\{l\}} & \rightarrow & \prod_{v \in C^{(1)}} H^{1}\left(K_{v}, T\right)\{l\} \\
\downarrow & \downarrow & & & \downarrow
\end{array} \\
& 0 \rightarrow{\overline{\mathcal{D}^{2}(U, \widehat{\mathcal{T}})\{l\}}}^{D} \rightarrow{\overline{H_{c}^{2}(U, \widehat{\mathcal{T}})\{l\}}}^{D} \rightarrow\left[\bigoplus_{v \in C^{(1)}} H^{1}\left(K_{v}, \widehat{\mathcal{T}}\right)\{l\}\right]^{D}
\end{aligned}
$$

qui induit donc un isomorphisme de groupes finis

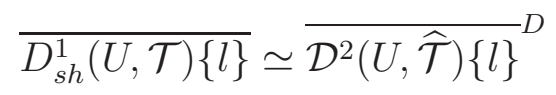

soit encore une dualité parfaite de groupes finis

$$
\overline{D_{\text {sh }}^{1}(U, \mathcal{T})\{l\}} \times \overline{\mathcal{D}^{2}(U, \widehat{\mathcal{T}})\{l\}} \rightarrow \mathbb{Q}_{l} / \mathbb{Z}_{l}(-1) .
$$

On a vu plus haut qu'on pouvait choisir $U$ de sorte que

$$
\mathcal{D}^{2}(U, \widehat{\mathcal{T}})\{l\} \simeq \amalg^{2}(K, \widehat{T})\{l\},
$$

et de même pour tout ouvert non vide $V \subset U$.

Le groupe fini $\amalg^{1}(K, T)\{l\}$ est la limite inductive des $D_{s h}^{1}(V, \mathcal{T})\{l\}$ pour $V \subset U$. Comme l'image d'un groupe divisible est un groupe divisible, $\amalg^{1}(K, T)\{l\}$ est aussi la limite inductive des groupes $\overline{D_{s h}^{1}(V, \mathcal{T})\{l\}}$.

Ceci démontre :

Pour tout $l$ premier, on a une dualité parfaite de groupes finis entre $\amalg^{1}(K, T)\{l\}$ et $\overline{\amalg^{2}(K, \widehat{T})\{l\}}$ par son sous-groupe divisible maximal.

Le groupe $\amalg^{1}(K, T) \subset H^{1}(K, T)$ est annulé par multiplication par le degré de toute extension de corps de $K$ déployant le $K$-tore $T$, d'où on déduit le résultat. 
Remarque 7.4. - L'exemple 2.10 montre que $\amalg^{1}(K, T)$ peut être non nul (un exemple plus explicite est développé dans la section 8.3). Montrons par ailleurs qu'il existe un réseau $\widehat{T}$ avec $\amalg^{1}(K, \widehat{T}) \neq 0$. Soit $E / \mathbb{C}$ une courbe elliptique. Soit $K=\mathbb{C}((t))(E)$. D'après le corollaire 2.4 on a $\amalg^{1}(K, \mathbb{Q} / \mathbb{Z}) \simeq$ $(\mathbb{Q} / \mathbb{Z})^{2}$. On a donc $\amalg^{2}(K, \mathbb{Z})=(\mathbb{Q} / \mathbb{Z})^{2}$. On part d'une classe $\xi$ non nulle dans $\amalg^{2}(K, \mathbb{Z})$. Il existe une extension $L$ finie galoisienne, de groupe $G$, telle que $\xi$ s'annule dans $H^{2}(L, \mathbb{Z})$.

La flèche $\mathbb{Z} \rightarrow \mathbb{Z}[G]$ définie par la norme $N_{G}=\sum_{g \in G} g$ induit une suite exacte de $G$-modules

$$
0 \rightarrow \mathbb{Z} \rightarrow \mathbb{Z}[G] \rightarrow \widehat{T} \rightarrow 0
$$

où $\widehat{T}$ est un réseau.

On déduit de cette suite un plongement

$$
\amalg^{1}(K, \widehat{T}) \hookrightarrow \amalg^{2}(K, \mathbb{Z}),
$$

et $\xi \in \amalg^{2}(K, \mathbb{Z})$ est dans l'image de $\amalg^{1}(K, \widehat{T})$, qui est donc non nul.

\section{Lien avec l'obstruction de réciprocité}

On discute ici l'analogue du paragraphe 5 de [17], et de considérations classiques sur les corps de nombres (voir [30, Chap. 6]).

Soient $K$ un corps, $G=\operatorname{Gal}(\bar{K} / K)$ et $Y$ un espace principal homogène d'un $K$-tore $T$. Comme on a $\operatorname{Pic}(\bar{Y})=0$, de la suite spectrale

$$
E_{2}^{p q}=H^{p}\left(K, H^{q}\left(\bar{Y}, \mathbb{G}_{m}\right)\right) \Longrightarrow H^{n}\left(Y, \mathbb{G}_{m}\right)
$$

on tire un isomorphisme

$$
\left.H^{2}\left(G, \bar{K}[Y]^{\times}\right)\right) \stackrel{\sim}{\rightarrow} \operatorname{Br}_{1}(Y) .
$$

où $\operatorname{Br}_{1}(Y)=\operatorname{Ker}[\operatorname{Br}(Y) \rightarrow \operatorname{Br}(\bar{Y})]$. On a la suite exacte de $G$-modules

$$
0 \rightarrow \bar{K}^{\times} \rightarrow \bar{K}[Y]^{\times} \rightarrow \widehat{T} \rightarrow 0 .
$$

On a donc des suites exactes

$$
\operatorname{Br}(K) \rightarrow \operatorname{Br}_{1}(Y) \rightarrow H^{2}(G, \widehat{T}) \rightarrow H^{3}\left(G, \bar{K}^{\times}\right) .
$$

Si l'on a $\operatorname{cd}(K) \leq 2$, alors on a $H^{3}\left(G, \bar{K}^{\times}\right)=0$.

Soit $k=\mathbb{C}((t))$. Supposons désormais que $K=k(C)$ est le corps des fonctions d'une $k$-courbe $C$ projective, lisse, géométriquement connexe. On 
note $\Omega$ l'ensemble des points fermés de $C$. Le corps $K$ et ses complétés $K_{v}$ aux points fermés $v \in \Omega$ sont de dimension cohomologique 2 .

On renvoie à la Proposition 2.1 pour les énoncés suivants. Pour chaque place $v$, on a $\operatorname{Br}\left(K_{v}\right) \stackrel{\widetilde{\Im}}{\rightarrow} H^{1}\left(\kappa_{v}, \mathbb{Q} / \mathbb{Z}\right) \simeq \mathbb{Q} / \mathbb{Z}(-1)$. On a une suite exacte

$$
0 \rightarrow \operatorname{Br}(C) \rightarrow \operatorname{Br}(K) \rightarrow \bigoplus_{v \in \Omega} \operatorname{Br}\left(K_{v}\right) \rightarrow \mathbb{Q} / \mathbb{Z}(-1) \rightarrow 0 .
$$

La nullité de l'application composée

$$
\operatorname{Br}(K) \rightarrow \bigoplus_{v \in \Omega} \operatorname{Br}\left(K_{v}\right) \rightarrow \mathbb{Q} / \mathbb{Z}(-1)
$$

est un cas particulier de loi de réciprocité.

8.1. Sous-groupes du groupe de Brauer. - Soient $Y$ une $K$-variété lisse géométriquement intègre et $Y_{c}$ une $K$-compactification lisse. Pour $v \in \Omega$, on note $Y_{v}=Y \times_{K} K_{v}$.

Le groupe $\operatorname{Br}_{1}(Y)=\operatorname{ker}[\operatorname{Br} Y \rightarrow \operatorname{Br} \bar{Y}]$ contient les sous-groupes suivants :

(a) Le groupe $\operatorname{Br}\left(Y_{c}\right)=\operatorname{Br}_{1}\left(Y_{c}\right) \subset \operatorname{Br}_{1}(Y)$.

(b) Le groupe $B(Y) \subset \operatorname{Br}_{1}(Y)$ formé des éléments de $\operatorname{Br}_{1}(Y)$ dont l'image dans $\operatorname{Br}_{1}\left(Y_{v}\right)$ appartient à l'image de $\operatorname{Br}\left(K_{v}\right)$ pour toute place $v \in C^{(1)}$.

(c) Le groupe $B_{\omega}(Y) \subset \operatorname{Br}_{1}(Y)$ formé des éléments de $\operatorname{Br}_{1}(Y)$ dont l'image dans $\operatorname{Br}_{1}\left(Y_{v}\right)$ appartient à l'image de $\operatorname{Br}\left(K_{v}\right)$ pour presque toute place $v \in C^{(1)}$. Un argument de bonne réduction, et la nullité des groupes $\operatorname{Br}\left(O_{v}\right)$ pour $O_{v}$ complété de l'anneau local en $v \in C$, de corps résiduel une extension finie de $\mathbb{C}((t))$, montre que $B_{\omega}(Y)$ est formé des éléments de $\operatorname{Br}_{1}(Y)$ dont l'image est nulle dans $\operatorname{Br}_{1}\left(Y_{v}\right)$ pour presque toute place $v \in C^{(1)}$.

On note $\operatorname{Br}_{a} Y$ le quotient de $\operatorname{Br}_{1} Y$ par l'image de $\operatorname{Br} K$. On note $\operatorname{Br}_{a} Y_{c}$ le quotient de $\operatorname{Br}_{1} Y_{c}$ par l'image de $\operatorname{Br} K$.

Considérons le cas où $Y$ est un espace principal homogène sous un $K$-tore $T$. Pour presque toute place $v$, le groupe de décomposition de l'extension minimale déployant le $K$-tore $T$ est cyclique (voir le paragraphe 1). De plus pour presque toute place $v$ on a $Y_{v} \simeq T_{v}$. Le groupe de Brauer d'une compactification lisse d'un tore déployé par une extension cyclique du corps de base est réduit au groupe de Brauer du corps de base ([28], Th. 9.2.ii), résultat dû aussi à Voskrensenskiǔ, cf. [32] Chap. 4, Section 11.6, Cor. 3 page 122). Le groupe $\operatorname{Br}\left(Y_{c}\right)=\operatorname{Br}_{1}\left(Y_{c}\right)$ est donc un sous-groupe du groupe $B_{\omega}(Y)$. 
On a le diagramme :

$$
\begin{array}{rlll}
B(Y) & \subset & B_{\omega}(Y) \subset \operatorname{Br}_{1}(Y) \\
\uparrow & & \uparrow \subset \\
\operatorname{Br}(K) & \rightarrow & \operatorname{Br}\left(Y_{c}\right) .
\end{array}
$$

Par passage au quotient par l'image de $\operatorname{Br}(K)$ dans $B(Y)$, resp. dans $B_{\omega}(Y)$, on définit le groupe $\mathrm{Б}(Y)$, resp. le groupe $\mathrm{Б}_{\omega}(Y)$, et on obtient le diagramme :

$$
\begin{gathered}
\mathrm{B}(Y) \subset \quad \mathrm{B}_{\omega}(Y) \subset \operatorname{Br}_{a}(Y) \\
\uparrow \subset \\
\\
\operatorname{Br}_{a}\left(Y_{c}\right)
\end{gathered}
$$

D'après les préliminaires de ce paragraphe, on a un diagramme commutatif de suites exactes

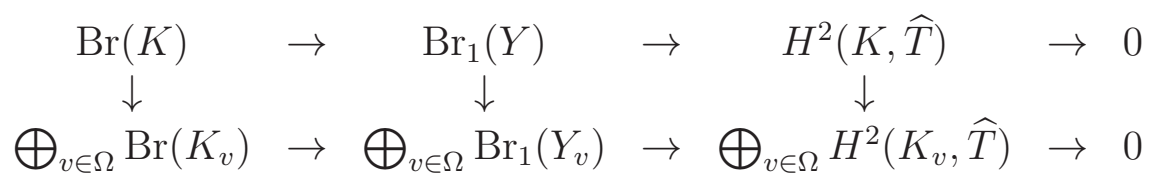

On a donc un isomorphisme $\mathrm{B}(Y) \stackrel{\simeq}{\rightarrow} \amalg^{2}(K, \widehat{T})$ et un isomorphisme $\mathrm{Б}_{\omega}(Y) \stackrel{\sim}{\rightarrow} \amalg_{\omega}^{2}(K, \widehat{T})$. Pour $i \in \mathbb{N}$, et un module galoisien $M$ sur le corps $K$, on note $\amalg_{\text {cyc }}^{i}(K, M)$ le noyau de la restriction

$$
H^{i}(G, M) \rightarrow \prod_{g \in G} H^{i}(<g>, M)
$$

aux sous-groupes fermés procycliques. D'après [8, Prop. 9.5], pour $T_{c}$ une compactification lisse de $T$, on a $\operatorname{Br}\left(T_{c}\right) / \operatorname{Br}(K) \simeq \amalg_{\text {cyc }}^{2}(K, \widehat{T})$. Comme $\operatorname{cd}(K) \leq 2$, d'après [28, Lemme 6.8] et [3, Prop. 2.2], il existe un isomorphisme naturel $\mathrm{Br}_{1}(Y) \stackrel{\simeq}{\rightarrow} \mathrm{Br}_{1}(T)$ compatible aux isomorphismes $\operatorname{Br}_{a}(Y) \stackrel{\simeq}{\rightarrow} H^{2}(K, \widehat{T})$ et $\operatorname{Br}_{a}(T) \stackrel{\simeq}{\rightarrow} H^{2}(K, \widehat{T})$, et induisant un isomorphisme $\mathrm{Br}_{a}\left(Y_{c}\right) \stackrel{\simeq}{\rightarrow} \mathrm{Br}_{a}\left(T_{c}\right)$.

On obtient ainsi un isomorphisme $\operatorname{Br}_{a}\left(Y_{c}\right) \stackrel{\simeq}{\rightarrow} \amalg_{\text {cyc }}^{2}(K, \widehat{T})$. Le diagramme ci-dessus se récrit alors :

$$
\begin{aligned}
& \amalg^{2}(K, \widehat{T}) \subset \amalg_{\omega}^{2}(K, \widehat{T}) \quad \subset H^{2}(K, \widehat{T}) \\
& \uparrow \subset \\
& \amalg_{\text {сус }}^{2}(K, \widehat{T})
\end{aligned}
$$

Remarques 8.1. - À la différence du cas où $K$ est un corps global:

(i) Même si $Y$ a des points dans tous les $K_{v}$ il n'est pas clair que $\operatorname{Br}(K)$ s'injecte dans $\operatorname{Br}(Y)$; 
(ii) Le groupe $\amalg_{\text {cyc }}^{2}(K, \widehat{T})$ est fini (propriété valable sur tout corps), mais les groupes $\mathrm{B}(Y)=\amalg^{2}(K, \widehat{T})$ et $\mathrm{Б}_{\omega}(Y)=\amalg_{\omega}^{2}(K, \widehat{T})$ peuvent être infinis. Pour $Y=T=\mathbb{G}_{m}$, on a

$$
\amalg^{2}(K, \mathbb{Z})=\amalg^{1}(K, \mathbb{Q} / \mathbb{Z})=\amalg_{\omega}^{1}(K, \mathbb{Q} / \mathbb{Z})=H^{1}\left(C_{0}, \mathbb{Q} / \mathbb{Z}\right),
$$

où $C_{0}$ est la courbe sur $\mathbb{C}$ fibre spéciale d'un modèle minimal de $C$ au-dessus de $\mathbb{C}[[t]]$ (voir le corollaire 2.5 et la démonstration du corollaire 2.6).

(iiii) Un élément de $B_{\omega}(Y)$, ou même de $B(Y)$, n'est pas nécessairement dans $\operatorname{Br}\left(Y_{c}\right)$.

8.2. Accouplement avec les points adéliques. - Soit $Y$ une $k$-variété lisse géométriquement intègre, possédant des $K_{v}$-points pour tout $v \in \Omega$. Soit $Y_{c}$ une $K$-compactifcation lisse de $Y$.

On a les inclusions

$$
Y\left(\mathbb{A}_{K}\right) \subset \prod_{v} Y\left(K_{v}\right) \subset \prod_{v} Y_{c}\left(K_{v}\right)=Y_{c}\left(\mathbb{A}_{K}\right) .
$$

En utilisant les isomorphismes $\partial_{v}: \operatorname{Br}\left(K_{v}\right) \stackrel{\widetilde{ }}{\rightarrow} \mathbb{Q} / \mathbb{Z}(-1)$, on peut définir plusieurs accouplements à la Brauer-Manin

$$
\begin{gathered}
Y\left(\mathbb{A}_{K}\right) \times \operatorname{Br}(Y) \rightarrow \mathbb{Q} / \mathbb{Z}(-1) \\
\prod_{v} Y\left(K_{v}\right) \times B_{\omega}(Y) \rightarrow \mathbb{Q} / \mathbb{Z}(-1) \\
\prod_{v} Y_{c}\left(K_{v}\right) \times B(Y) \rightarrow \mathbb{Q} / \mathbb{Z}(-1)
\end{gathered}
$$

envoyant le couple formé d'une famille $\left\{P_{v}\right\}_{v \in \Omega}$ et d'un élément $\alpha$ sur la somme (qui n'a qu'un nombre fini de termes non nul)

$$
\sum_{v} \partial_{v}\left(\alpha\left(P_{v}\right)\right) \in \mathbb{Q} / \mathbb{Z}(-1)
$$

Comme on a le complexe

$$
\operatorname{Br}(K) \rightarrow \bigoplus_{v \in \Omega} \operatorname{Br}\left(K_{v}\right) \rightarrow \mathbb{Q} / \mathbb{Z}(-1),
$$

ces accouplements ne dépendent que des quotients $\operatorname{Br}(Y) / \operatorname{Br}(K)$, resp. $\mathrm{Б}_{\omega}(Y)$, resp. Б $(Y)$.

Le dernier accouplement ne dépend pas du terme de gauche. En effet un élément de $B(Y)$ provient d'un élément $\alpha \in \operatorname{Br}(Y)$ tel que pour tout $v \in \Omega$ il existe $\beta_{v} \in \operatorname{Br}\left(K_{v}\right)$ dont l'image dans $\operatorname{Br}\left(Y_{v}\right)$ coöncide avec l'image de $\alpha$, 
la famille $\left\{\beta_{v}\right\}$ étant définie à addition de l'image diagonale d'un élément de $\operatorname{Br}(K)$. La somme

$$
\sum_{v} \partial_{v}\left(\alpha\left(P_{v}\right)\right)=\sum_{v} \partial_{v}\left(\beta_{v}\right)
$$

est alors clairement indépendante de la famille $\left\{P_{v}\right\}$. Ainsi $Y$ définit un homomorphisme

$$
\rho_{Y}: \mathrm{B}(Y) \rightarrow \mathbb{Q} / \mathbb{Z}(-1) .
$$

On note $Y\left(\mathbb{A}_{K}\right)^{\operatorname{Br}(Y)}$ le noyau à gauche de la première flèche, et on emploie des notations semblables pour les autres accouplements. Du complexe ci-dessus on tire les inclusions

$$
Y(K) \subset Y\left(\mathbb{A}_{K}\right)^{\operatorname{Br}(Y)} \subset\left[\prod_{v \in \Omega} Y\left(K_{v}\right)\right]^{\mathrm{E} \omega}(Y) \subset\left[\prod_{v \in \Omega} Y\left(K_{v}\right)\right]^{\mathrm{D}(Y)} \subset Y_{c}\left(\mathbb{A}_{K}\right)^{\mathrm{E}(Y)} .
$$

Proposition 8.2. - Soit T un K-tore et $Y$ un K-espace principal homogène possédant des points dans tous les $K_{v}$. Soit $\xi \in \amalg^{1}(K, T)$ la classe de $Y$. Soit $\beta \in \mathrm{E}(Y)$ et $\gamma \in \amalg^{2}(K, \widehat{T})$ son image par l'isomorphisme $\mathrm{D}(Y) \stackrel{\sim}{\rightarrow} \amalg^{2}(K, \widehat{T})$. On a l'égalité :

$$
\rho_{Y}(\beta)=<\xi, \gamma>\in \mathbb{Q} / \mathbb{Z}(-1),
$$

où $<\xi, \gamma>$ est la valeur sur $(\xi, \gamma)$ de l'accouplement

$$
\amalg^{1}(K, T) \times \amalg^{2}(K, \widehat{T}) \rightarrow \mathbb{Q} / \mathbb{Z}(-1)
$$

qui fait l'objet du théorème 7.2

Démonstration. - La preuve est essentiellement identique à celle de [16], sections 3 et 4 (qui vaut sur un corps de nombres, pour un 1-motif quelconque à la place du tore $T$ ); nous nous bornons ici à en rappeler les grandes lignes. On commence par étendre $T$ en un $U$-tore $\mathcal{T}$, et on relève la classe $\xi \in \amalg^{1}(T)$ en une classe $\xi_{U} \in H^{1}(U, \mathcal{T})$ pour $U$ assez petit. De même on peut relever $\gamma$ en $\gamma_{U} \in H_{c}^{2}(U, \widehat{\mathcal{T}})$. La proposition 3.3. de [16] s'applique encore dans notre cadre, avec une preuve analogue (si ce n'est qu'il faut remplacer, dans le diagramme (7) de [16], le groupe Brk par son image dans $\left.\operatorname{Br}_{1} Y\right)$ et donne

$$
\rho_{Y}(\beta)=\mathcal{E}_{Y} \cup \gamma_{U}
$$

où $\mathcal{E}_{Y} \in \operatorname{Ext}_{U}^{1}\left(\widehat{\mathcal{T}}, \mathbb{G}_{m}\right)$ est la classe d'une extension dont la fibre générique sur Spec $K$ est celle de

$$
0 \rightarrow \bar{K}^{*} \rightarrow \bar{K}[Y]^{*} \rightarrow \bar{K}[Y]^{*} / \bar{K}^{*}=\widehat{T} \rightarrow 0 .
$$


Ensuite, les lemmes 2.3.7. et 2.4.3. de de [30] donnent que la classe de l'extension (8.1) dans $\operatorname{Ext}_{K}^{1}\left(\widehat{T}, \mathbb{G}_{m}\right)=H^{1}(K, T)$ est précisément celle de $\xi$. Quitte à rétrécir $U$, on peut donc supposer que $\mathcal{E}_{Y}=\xi_{U}$, ce qui donne le résultat d'après la définition de l'accouplement du théorème 7.2 .

On en déduit l'analogue du théorème de Voskrenskiǐ-Sansuc sur les corps de nombres et du théorème 5.1 de [17] sur le corps de fonctions d'une courbe au-dessus d'un corps $p$-adique :

Corollaire 8.3. - Soit $Y$ un espace principal homogène sous un K-tore. Supposons que $Y$ possède des points dans tous les $K_{v}$. L'obstruction à l'existence d'un point rationnel sur $Y$ associée au groupe $B(Y)$ et à la loi de réciprocité sur $C$ est la seule obstruction à l'existence d'un point rationnel sur $Y$.

Démonstration. - L'hypothèse assure que l'application $\rho_{Y}$ est nulle. La proposition 8.2 donne alors que la classe de $Y$ dans $\amalg^{1}(K, T)$ est orthogonale à $\amalg^{2}(K, \widehat{T})$ pour un accouplement qui, d'après le le théorème 7.2 , est non dégénéré sur $\amalg^{1}(K, T)$.

8.3. Un exemple.- Nous présentons ici avec plus de détails un exemple donné dans [6], Prop. 3.2. Cet exemple illustre la proposition 8.2 et le corollaire 8.3 ci-dessus.

Soient $k=\mathbb{C}((t))$ et $K=\mathbb{C}((t))(x)$. On considère la $K$-variété $E$ définie par l'équation

$$
\left(X_{1}^{2}-x Y_{1}^{2}\right)\left(X_{2}^{2}-(1+x) Y_{2}^{2}\right)\left(X_{3}^{2}-x(1+x) Y_{3}^{2}\right)=t .
$$

Assertion I. Si P est un point fermé de $\mathbf{P}_{k}^{1}$ l'un de $x, x+1, x(x+1)$ est un carré dans le complété $K_{P}$, et donc $E\left(K_{P}\right) \neq \emptyset$ et $E_{K_{P}}$ est une variété $K_{P}$-rationnelle.

Calculons les valeurs dans $K_{P}$ pour tout point fermé $P$.

Au point à l'infini, $x(x+1)$ est un carré. Mais ni $x$ ni $x+1$ ne le sont, car leur valuation est -1 .

Au point $x=0, x+1$ est un carré car en réduction il vaut 1 . Ni $x$ ni $x(x+1)$ ne sont des carrés, car leur valuation est 1 .

Au point $x=-1, x$ est un carré car -1 est un carré dans $k$. Ni $x+1$ ni $x(x+1)$ ne sont des carrés, car leur valuation est 1 . 
Soit $P$ un autre point fermé de $\mathbf{P}_{k}^{1}$. Alors $x, x+1$ et $x(x+1)$ sont des unités en $P$.

Soit $\xi$ la classe de $x$ dans le corps résiduel $\kappa(P)$. On a donc $\xi \neq 0$ et $\xi \neq-1$. Soit $v$ la valuation sur le corps résiduel $\kappa(P)$, extension finie de $k=\mathbb{C}((t))$.

Si $v(\xi)<0$, alors $\xi(\xi+1)$ est un carré dans $\kappa(P)$ et donc $x(x+1)$ est un carré dans $K_{P}$.

Si $v(\xi)>0$ alors $\xi+1$ est un un carré dans $\kappa(P)$ et donc $x+1$ est un carré dans $K_{P}$.

Si $v(\xi)=0$ et $v(\xi+1)=0$ alors chacun de $\xi, \xi+1$ et $\xi(\xi+1)$ est une unité dans $\kappa(P)$. Chacun est donc un carré dans $\kappa(P)$. Chacun de $x, x+1$, $x(x+1)$ est donc un carré dans $K_{P}$.

Si $v(\xi)=0$ et $v(\xi+1)>0$, alors $\xi$ est est un carré dans $\kappa(P)$ et donc $x$ est un carré dans $K_{P}$.

Ainsi pour toute valeur de $c \in K^{\times}$, en particulier pour $c=t$, l'équation

$$
\left(X_{1}^{2}-x Y_{1}^{2}\right)\left(X_{2}^{2}-(1+x) Y_{2}^{2}\right)\left(X_{3}^{2}-x(1+x) Y_{3}^{2}\right)=c
$$

a des solutions dans tous les $K_{P}$ pour $P$ point fermé de $\mathbf{P}_{k}^{1}$.

Assertion II.

Soit $w$ la valuation de $K=k\left(\mathbf{P}^{1}\right)$ associée au point générique de la fibre spéciale de $\mathbf{P}_{\mathbb{C}[t] t^{1}}^{1}$. On a $E\left(K_{w}\right)=\emptyset$, et donc $E(K)=\emptyset$.

L'élément $t$ est une uniformisante pour $w$, le corps résiduel de $w$ est le corps $\mathbb{C}(x)$. Chacun des $x, x+1, x(x+1)$ est une $w$-unité, et n'est pas un carré dans le corps résiduel de $w$. On en déduit que pour toute valeur $c$ non nulle de

$$
\left(X_{1}^{2}-x Y_{1}^{2}\right)\left(X_{2}^{2}-(1+x) Y_{2}^{2}\right)\left(X_{3}^{2}-x(1+x) Y_{3}^{2}\right)
$$

avec les $X_{i}, Y_{i}$ dans $k(x)$, on a $w(c)$ paire. Mais $w(t)=1$. Donc $t$ n'est pas représenté par ce produit sur le complété $K_{w}$ de $K$ en le point générique de la fibre spéciale, et donc pas sur $K$.

Assertion III. Cet exemple s'explique par la loi de réciprocité sur la courbe $\mathbf{P}_{k}^{1}$.

Notons $Z / K$ une compactification lisse de la $K$-variété $E$ d'équation

$$
\left(X_{1}^{2}-x Y_{1}^{2}\right)\left(X_{2}^{2}-(1+x) Y_{2}^{2}\right)\left(X_{3}^{2}-x(1+x) Y_{3}^{2}\right)=t .
$$

On sait [3] que le groupe de Brauer d'un modèle projectif et lisse $Z$ est engendré modulo $\operatorname{Br}(K)$ par l'élément d'ordre 2 de $\operatorname{Br}(K(Z))$ défini par

$$
\begin{gathered}
A=\left(X_{1}^{2}-x Y_{1}^{2}, x+1\right)=\left(X_{1}^{2}-x Y_{1}^{2}, x(x+1)\right)= \\
\left(X_{3}^{2}-x(1+x) Y_{3}^{2}, x+1\right)+(t, x+1) .
\end{gathered}
$$


Pour $P$ parcourant les points fermés de $\mathbf{P}_{k}^{1}$, on a vu que $Z_{K_{P}}$ est $K_{P^{-}}$ rationnel. Ceci implique $\operatorname{Br} Z_{K_{P}}=\operatorname{Br} K_{P}$. La valeur de $A\left(M_{P}\right) \in \operatorname{Br}\left(K_{P}\right)$ ne dépend donc pas du choix de $M_{P} \in Z\left(K_{P}\right)$.

On a $H^{1}(k, \mathbb{Z} / 2)=k^{\times} / k^{\times 2} \simeq \mathbb{Z} / 2$. On a obstruction de réciprocité si l'on a :

$$
\sum_{P} \operatorname{Cores}_{k(P) / k}\left(\delta_{P}\left(A\left(M_{P}\right)\right) \neq 0 \in \mathbb{Z} / 2\right.
$$

Au point $P$ défini par $x=0, x+1$ est un carré, donc $A_{K_{P}}=0$.

Au point $P$ défini par $x=\infty, x(x+1)$ est un carré, donc $A_{K_{P}}=0$.

Au point défini par $x=-1, x$ est un carré, on trouve $A_{K_{P}}=(t, x+1) \in$ $\operatorname{Br}\left(K_{P}\right)$, et donc

$$
\operatorname{Cores}_{k(P) / k} \delta_{P}\left(A\left(M_{P}\right)\right)=1 \in \mathbb{Z} / 2 .
$$

Considérons les points fermés $P \in \mathbf{P}_{k}^{1}$ où $x$ et $x+1$ sont des unités. Soit $\xi$ la classe de $x$ dans le corps résiduel $\kappa(P)$. Soit $v$ la valuation sur le corps résiduel $\kappa(P)$, extension finie de $k=\mathbb{C}((t))$.

Si $v(\xi)<0$, alors $x(x+1)$ est un carré dans $K_{P}$, donc $A_{K_{P}}=0$.

Si $v(\xi)>0$, alors $x+1$ est un carré dans $K_{P}$, donc $A_{K_{P}}=0$.

Si $v(\xi)=0$, alors $x$ est un carré dans $K_{P}$, donc $A_{K_{P}}=(t, x+1)$, avec $t$ et $x+1$ unités en $P$, et donc $\partial_{P}\left(A_{K_{P}}\right)=0$.

On obtient donc

$$
\sum_{P} \operatorname{Cores}_{k(P) / k}\left(\delta_{P}\left(A\left(M_{P}\right)\right)=1 \in \mathbb{Z} / 2 .\right.
$$

Il y a donc obstruction de réciprocité sur la courbe $\mathbf{P}_{k}^{1}$, et $Z(K)=\emptyset$.

Dans cet exemple, $E$ est un espace principal homogène sous le $K$-tore $T$ obtenu en remplaçant $t$ par 1 dans le membre de droite de l'équation de $E$. La classe de $A$ dans $\operatorname{Br}(Z) \subset \operatorname{Br}(E)$ est constante en tout $v \in \Omega$. Elle définit donc un élément de $B(E)$, dont l'image dans $\mathrm{S}(E)$ définit un élément $\gamma \in$ $\amalg^{2}(K, \widehat{T})$. La proposition 8.2 permet de traduire le calcul fait ci-dessus en termes de l'accouplement

$$
\amalg^{1}(K, T) \times \amalg^{2}(K, \widehat{T}) \rightarrow \mathbb{Q} / \mathbb{Z}(-1) .
$$

Remarques 8.4. - Dans l'exemple 2.10 (voir aussi [6, Exemple 2.6]), on a exhibé des espaces principaux homogènes $Y$ sous un $K$-tore $T$, avec $\prod_{v} Y\left(K_{v}\right) \neq \emptyset$ tels que $\operatorname{Br}_{a}\left(Y_{c}\right)=0$ et $Y(K)=\emptyset$. D'après le corollaire 8.3 , 
on doit pouvoir expliquer cet exemple au moyen d'un élément explicite de $B(Y)$, ou de $\amalg^{2}(K, \widehat{T})$, mais cela ne semble pas immédiat.

Les tores $T$ dans les exemples mentionnés sont $K$-rationnels. Pour un tore $K$-rationnel sur un corps $K=\mathbb{C}((t))(C)$, c'est une conséquence de théorèmes d'Harbater, Hartmann et Krashen, et de travaux antérieurs, que le principe local-global, par rapport à l'ensemble de toutes les valuations discrètes de rang 1 , vaut pour les espaces principaux homogènes de $K$-groupes linéaires connexes $K$-rationnels [19, Cor. 8.11]. On doit donc pouvoir aussi établir $Y(K)=\emptyset$ en considérant un complété de $K$ en une place non triviale sur $\mathbb{C}((t))$, et c'est de fait facile à établir dans le cas présent.

\section{Approximation faible}

Soient $k=\mathbb{C}((t))$ et $K=k(C)$ le corps des fonctions d'une courbe.

Comme on a vu au début de l'article, le corps $K$ est un corps "de type arithmétique" au sens de [25, §7].

9.1. Approximation faible pour les tores. - Etant donné $T$ un $K$-tore, on peut considérer le problème d'approximation faible par rapport aux places $v \in C^{(1)}$. On note $\overline{T(K)}$ l'adhérence de $T(K)$ dans le produit direct des $T\left(K_{v}\right)$. De même, si $S$ est un sous-ensemble fini non vide de $C^{(1)}$, on note $\overline{T(K)}_{S}$ l'adhérence de $T(K)$ dans $\prod_{v \in S} T\left(K_{v}\right)$. Le défaut d'approximation faible pour $T$ est le conoyau de l'injection diagonale $\overline{T(K)} \rightarrow \prod_{v \in \Omega_{K}} T\left(K_{v}\right)$, ce dernier groupe étant muni de la topologie produit des topologies $v$-adiques.

Proposition 9.1. - Soit

$$
1 \rightarrow Q \rightarrow R \rightarrow T \rightarrow 1
$$

une résolution flasque de $T$. Alors :

a) Les groupes $H^{1}\left(K_{v}, Q\right)$ sont nuls pour presque toute place $v \in C^{(1)}$, et le groupe $H^{1}(K, Q)$ est fini.

b) Le défaut d'approximation faible pour $T$ est fini, et isomorphe au conoyau de la flèche diagonale $H^{1}(K, Q) \rightarrow \bigoplus_{v \in \Omega_{K}} H^{1}\left(K_{v}, Q\right)$, qui est un homomorphisme de groupes finis.

Démonstration. - a) Soit $v$ une place de bonne réduction pour le $K$-tore flasque $Q$. Alors $H^{1}\left(O_{v}, Q\right)$ se surjecte sur $H^{1}\left(K_{v}, Q\right)[\mathbf{8}$, Thm. 2.2], mais $H^{1}\left(O_{v}, Q\right)=H^{1}\left(\kappa_{v}, Q\right)=0 \operatorname{car} \operatorname{cd}\left(\kappa_{v}\right)=1$. Finalement $H^{1}\left(K_{v}, Q\right)$ est nul en toute place de bonne réduction de $Q$. 
Il existe un ouvert $U \subset C$ sur lequel $Q$ s'étend en un $U$-tore flasque, notons-le $Q[\mathbf{8}$, Prop. 1.5].

La flèche $H^{1}(U, Q) \rightarrow H^{1}(K, Q)$ est surjective [8, Thm. 2.2]. Comme $H^{1}(K, Q)$ est annulé par un entier $n>0$, la flèche se factorise par $H^{1}(U, Q) / n$, qui est un sous-groupe du groupe $H^{2}\left(U,{ }_{n} Q\right)$, qui est fini (Lemme 4.4). Donc $H^{1}(K, Q)$ est fini.

b) Comme le tore $R$ est quasi-trivial, on a $H^{1}(K, R)=H^{1}\left(K_{v}, R\right)=0$ pour toute place $v$. On en déduit, pour tout sous-ensemble fini non vide $S$ de $C^{(1)}$, un diagramme commutatif à lignes exactes :

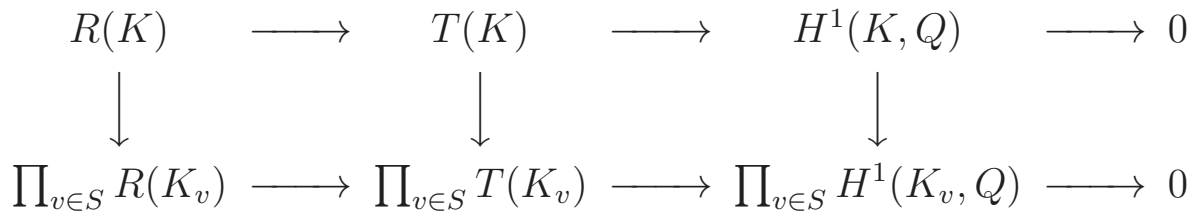

où les groupes sur la verticale de droite sont finis. Le tore quasi-trivial (donc $K$-rationnel) $R$ vérifie l'approximation faible. On en déduit une suite exacte

$$
0 \rightarrow \overline{T(K)}_{S} \rightarrow \prod_{v \in S} T\left(K_{v}\right) \rightarrow \operatorname{Coker}\left[H^{1}(K, Q) \rightarrow \bigoplus_{v \in S} H^{1}\left(K_{v}, Q\right)\right] .
$$

Comme $H^{1}\left(K_{v}, Q\right)=0$ en dehors de l'ensemble fini $S_{0}$ des places de mauvaise réduction de $Q$, on obtient le résultat.

Remarque 9.2. - Si le $K_{v}$-tore $T$ est déployé par une extension cyclique, alors $H^{1}\left(K_{v}, Q\right)=0$. On peut ainsi "diminuer" le nombre de places de mauvaise réduction à considérer. De fait, dans la présente situation, en une place de bonne réduction, le tore est bien déployé par une extension cyclique, car toute extension de $\mathbb{C}((t))$ est cyclique.

Soit $F$ un module galoisien fini sur $K=\mathbb{C}((t))(C)$. Il s'étend en faisceau étale $\mathcal{F}$ sur un ouvert non vide $U \subset C$. Pour tout entier $i \geq 0$, le produit restreint $\mathbf{P}^{i}(F) \subset \prod_{v \in C^{(1)}} H^{i}\left(K_{v}, F\right)$ est le sous-groupe formé des familles $\left\{\xi_{v}\right\}$ avec $\xi_{v} \in H^{i}\left(O_{v}, F\right)$ pour presque tout $v \in U$. Pour tout module galoisien $M$, et tout ensemble fini $S$ de $C^{(1)}$, on note $\amalg_{S}^{i}(M)$ le sous-groupe de $H^{i}(K, M)$ constitué des classes nulles en dehors de $S$.

En particulier le sous-groupe $\amalg_{\omega}^{i}(M)$ des classes nulles presque partout est la limite inductive (sur l'ensemble des $S$ finis) des $\amalg_{S}^{i}(M)$.

Proposition 9.3. - Soit $F$ un module galoisien fini sur $K=\mathbb{C}((t))(C)$, de dual $\widehat{F}$. 
Les applications de dualité locale

$$
H^{1}\left(K_{v}, F\right) \times H^{1}\left(K_{v}, \widehat{F}\right) \rightarrow \mathbb{Q} / \mathbb{Z}(-1)
$$

de la proposition 3.3 induisent des applications $\mathbf{P}^{1}(F) \rightarrow H^{1}(K, \widehat{F})^{D}$ et $\prod_{v \in S} H^{1}\left(K_{v}, F\right) \rightarrow \amalg_{S}^{1}(\widehat{F})^{D}$.

(a) On a une suite exacte de groupes abéliens

$$
H^{1}(K, F) \rightarrow \mathbf{P}^{1}(F) \rightarrow H^{1}(K, \widehat{F})^{D} .
$$

(b) Si S est un sous-ensemble fini non vide de $C^{(1)}$, on a une suite exacte de groupes abéliens

$$
H^{1}(K, F) \rightarrow \prod_{v \in S} H^{1}\left(K_{v}, F\right) \rightarrow \amalg_{S}^{1}(\widehat{F})^{D} \rightarrow \amalg^{1}(\widehat{F})^{D} \rightarrow 0 .
$$

Le groupe $H^{1}(K, \widehat{F})$ est en général infini. Dans la seconde suite, les groupes autres que $H^{1}(K, F)$ sont finis. La finitude de $\amalg_{S}^{1}(\widehat{F})$ résulte de la proposition 4.2 et du lemme 4.4.

Démonstration. - La démonstration est entièrement analogue à celle de [14, Prop. 2.1], avec la différence qu'ici le corps $K$ est de dimension cohomologique 2. On passe par la dualité d'Artin-Verdier (Prop. 5.1 ci-dessus) pour les faisceaux étales finis sur un ouvert de la courbe $C$ pour obtenir le premier énoncé. On en déduit le second en utilisant la dualité locale (Prop. 3.3), comme dans [14. Lemma 3.1].

Théorème 9.4. - Soit T un K-tore.

Soit $S$ un ensemble fini non vide de places de K. Alors on a une suite exacte de groupes abéliens

$$
0 \rightarrow \overline{T(K)}_{S} \rightarrow \prod_{v \in S} T\left(K_{v}\right) \rightarrow \amalg_{S}^{2}(\widehat{T})^{D} \rightarrow \amalg^{2}(\widehat{T})^{D} \rightarrow 0,
$$

l'application

$$
\prod_{v \in S} T\left(K_{v}\right) \rightarrow \amalg_{S}^{2}(\widehat{T})^{D}
$$

étant induite par les applications de dualité locale

$$
T\left(K_{v}\right) \times H^{2}\left(K_{v}, \widehat{T}\right) \rightarrow \mathbb{Q} / \mathbb{Z}(-1)
$$

de la proposition 3.4

Dans cette suite exacte, l'image de $\prod_{v \in S} T\left(K_{v}\right) \rightarrow \amalg_{S}^{2}(\widehat{T})^{D}$ est un groupe fini. 
Noter que l'application $\prod_{v \in S} T\left(K_{v}\right) \rightarrow \amalg_{S}^{2}(\widehat{T})^{D}$ est continue via la remarque 3.5 .

Démonstration. - On montre d'abord deux lemmes :

Lemme 9.5. — La suite de groupes abéliens

$$
T(K) \rightarrow \prod_{v \in S} T\left(K_{v}\right) \rightarrow \amalg_{S}^{2}(\widehat{T})^{D}
$$

est un complexe.

On note d'abord qu'on a des accouplements locaux

$$
H^{0}\left(K_{v}^{h}, T\right) \times H^{2}\left(K_{v}^{h}, \widehat{T}\right)=H^{2}\left(K_{v}, \widehat{T}\right) \rightarrow \operatorname{Br} K_{v}^{h}=\operatorname{Br} K_{v} \simeq \mathbb{Q} / \mathbb{Z}(-1)
$$

qui sont compatibles avec les accouplements analogues faisant intervenir $H^{0}\left(K_{v}, T\right)$ au lieu de $H^{0}\left(K_{v}^{h}, T\right)$. Il suffit donc de démontrer que la suite

$$
T(K) \rightarrow \prod_{v \in S} T\left(K_{v}^{h}\right) \rightarrow \amalg_{S}^{2}(\widehat{T})^{D}
$$

est un complexe, la dernière flèche étant induite par les accouplements locaux.

Soient $t \in T(K)$ et $\alpha \in \amalg_{S}^{2}(\widehat{T})$. Soit $U$ un ouvert affine non vide de $C$ tel que $T$ s'étende en un $U$-tore $\mathcal{T}$ avec de plus : $t \in \mathcal{T}(U)=H^{0}(U, \mathcal{T})$ et $\alpha$ se relève en un élément (encore noté $\alpha$ ) de $H^{2}(U, \widehat{\mathcal{T}})$. On peut supposer (quitte à restreindre $U$ et à augmenter $S$ ) que $S=C-U$. Soient $\left(t_{v}\right)$ l'image de $t$ dans $\prod_{v \in S} T\left(K_{v}^{h}\right)$ et $\theta$ l'image de $\left(t_{v}\right)$ dans $\amalg_{S}^{2}(\widehat{T})^{D}$. Alors

$$
\theta(\alpha)=\sum_{v \in S}\left\langle t_{v}, \alpha_{v}\right\rangle
$$

est aussi donné par l'accouplement d'Artin-Verdier $\langle u, \alpha\rangle$, où $u$ est l'image de $\left(t_{v}\right)$ dans $H_{c}^{1}(U, \mathcal{T})$ car on a compatibilité entre accouplement d'ArtinVerdier et accouplements locaux (lemme 6.1). Mais $u$ est nul via la suite de localisation [24, Prop. II.2.3 p. 166]

$$
H^{0}(U, \mathcal{T}) \rightarrow \bigoplus_{v \in S} H^{0}\left(K_{v}^{h}, T\right) \rightarrow H_{c}^{1}(U, \mathcal{T})
$$

Ceci démontre le premier lemme.

Lemme 9.6. — La suite de groupes abéliens

$$
0 \rightarrow \overline{T(K)}_{S} \rightarrow \prod_{v \in S} T\left(K_{v}\right) \rightarrow \amalg_{S}^{2}(\widehat{T})^{D}
$$


est exacte, et l'image de $\prod_{v \in S} T\left(K_{v}\right) \rightarrow \amalg_{S}^{2}(\widehat{T})^{D}$ est un groupe fini.

L'exactitude se montre comme dans le Th. 3.3. de [14], en se ramenant (par le lemme d'Ono) au cas où $T$ admet une résolution

$$
0 \rightarrow F \rightarrow R \rightarrow T \rightarrow 0
$$

avec $F$ fini et $R$ tore quasi-trivial. On a alors le diagramme commutatif (9.1)

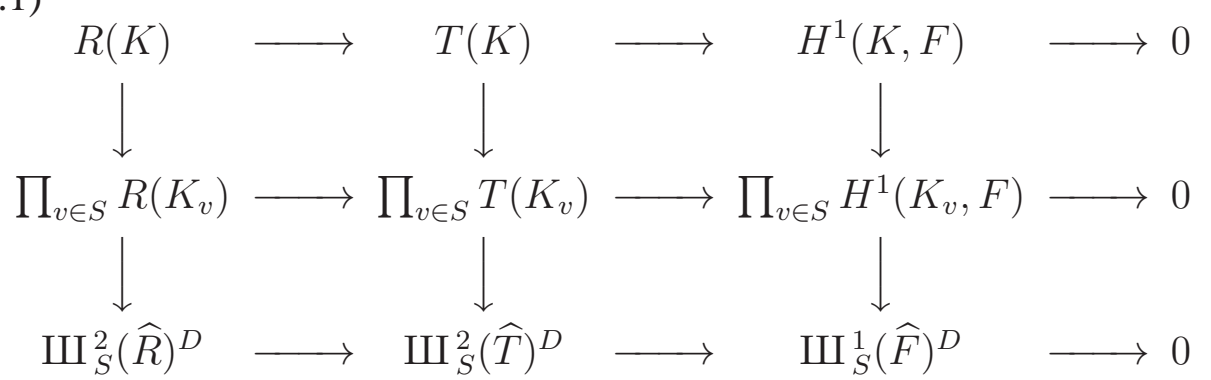

où les suites horizontales sont exactes, les suites verticales sont des complexes, et la suite verticale de droite est exacte par la proposition 9.3 .

Le tore $R$ est quasi-trivial. La proposition 2.6 montre que l'on a $\amalg_{S}^{2}(\widehat{R})=$ $\amalg^{2}(\widehat{R})$. Ceci implique que l'application $\prod_{v \in S} R\left(K_{v}\right) \rightarrow \amalg_{S}^{2}(\widehat{R})^{D}$ est nulle.

Du diagramme on conclut que la flèche $\prod_{v \in S} T\left(K_{v}\right) \rightarrow \amalg_{S}^{2}(\widehat{T})^{D}$ se factorise par le groupe fini $\prod_{v \in S} H^{1}\left(K_{v}, F\right)$.

Le tore $R$ étant quasitrivial satisfait l'approximation faible. L'adhérence de $T(K)$ dans $\prod_{v \in S} T\left(K_{v}\right)$ est le sous-groupe ouvert d'indice fini engendré par $T(K)$ et l'image de $\prod_{v \in S} R\left(K_{v}\right) \rightarrow \prod_{v \in S} T\left(K_{v}\right)$. Notons $A_{S}(T)$ le groupe fini quotient de $\prod_{v \in S} T\left(K_{v}\right)$ par l'adhérence de $T(K)$.

Du diagramme on déduit un homomomorphisme $A_{S}(T) \rightarrow \amalg_{S}^{2}(\widehat{T})^{D}$ qui composé avec $\amalg_{S}^{2}(\widehat{T})^{D} \rightarrow \amalg_{S}^{1}(\widehat{F})^{D}$ est injectif. Ainsi $A_{S}(T) \rightarrow \amalg_{S}^{2}(\widehat{T})^{D}$ est aussi injectif.

Remarque 9.7. - Conservons les notations de la démonstration. De la suite exacte

$$
0 \rightarrow \widehat{T} \rightarrow \widehat{R} \rightarrow \widehat{F} \rightarrow 0
$$

on déduit une suite exacte (définissant le groupe $B$ )

$$
0 \rightarrow \amalg_{S}^{1}(\widehat{F}) \rightarrow \amalg_{S}^{2}(\widehat{T}) \rightarrow \amalg_{S}^{2}(\widehat{R}) \rightarrow B \rightarrow 0,
$$

et l'on vérifie que groupe $B$ est annulé par le carré de l'ordre du groupe fini $\widehat{F}$. Comme $\widehat{R}$ est un module de permutation, la proposition 2.6 montre que 
l'on a

$$
\amalg^{2}(\widehat{R})=\amalg_{S}^{2}(\widehat{R})=\amalg_{\omega}^{2}(\widehat{R})
$$

et que ce groupe est divisible. Comme $B$ est annulé par un entier non nul, ceci implique $B=0$. La suite inférieure dans le diagramme (9.1) est donc exacte aussi à gauche. Le groupe $\amalg_{S}^{2}(\widehat{R})^{D}$ est sans torsion.

S'il en était besoin, ceci permettrait de donner une démonstration alternative du lemme 9.5, à savoir que la verticale médiane dans le diagramme (9.1) est un complexe. Soit en effet $\xi \in T(K)$. Soit $\rho \in \amalg_{S}^{2}(\widehat{T})^{D}$ son image par l'application composée. Comme la verticale de droite est un complexe, ceci implique qu $\rho=\sigma$ avec $\sigma$ dans le sous-groupe sans torsion $\amalg_{S}^{2}(\widehat{R})^{D} \subset \amalg_{S}^{2}(\widehat{T})^{D}$. Comme $H^{1}(K, F)$ est annulé par l'ordre $n$ de $F$, on voit que $n \xi$ est image de $\eta \in R(K)$. L'image de $\eta$ par l'application composée dans $\amalg_{S}^{2}(\widehat{R})^{D}$ est nulle. On a donc $n \sigma=0$ mais comme $\amalg_{S}^{2}(\widehat{R})^{D}$ est sans torsion, $\sigma=0$ et donc $\rho=0$.

Remarque 9.8. - On voit ainsi que l'adhérence du groupe $T(K)$ dans $\prod_{v \in S} T\left(K_{v}\right)$ a son image nulle dans $\amalg_{S}^{2}(\widehat{T})^{D}$. On aurait pu établir ce fait directement en rappelant que l'application $\prod_{v \in S} T\left(K_{v}\right) \rightarrow \prod_{v \in S} H^{2}\left(K_{v}, \widehat{T}\right)^{D}$ est continue. On obtient ainsi une démonstration qui n'utilise pas l'égalité $\amalg_{S}^{2}(\widehat{R})=\amalg^{2}(\widehat{R})$.

Pour finir la preuve du théorème, il faut établir l'exactitude des trois derniers termes

$$
\prod_{v \in S} T\left(K_{v}\right) \rightarrow \amalg_{S}^{2}(\widehat{T})^{D} \rightarrow \amalg^{2}(\widehat{T})^{D} \rightarrow 0 .
$$

Celle-ci se montre comme dans le Théorème 3.3. de [14].

On part de la suite exacte évidente de groupes discrets de torsion

$$
0 \rightarrow \amalg^{2}(\widehat{T}) \rightarrow \amalg_{S}^{2}(\widehat{T}) \rightarrow \bigoplus_{v \in S} H^{2}\left(K_{v}, \widehat{T}\right) .
$$

Par dualité on obtient une suite exacte de groupes compacts

$$
\prod_{v \in S} H^{2}\left(K_{v}, \widehat{T}\right)^{D} \rightarrow \amalg_{S}^{2}(\widehat{T})^{D} \rightarrow \amalg^{2}(\widehat{T})^{D} \rightarrow 0,
$$

où les flèches sont continues.

Par dualité locale, on a une application

$$
\theta: \prod_{v \in S} T\left(K_{v}\right) \rightarrow \prod_{v \in S} H^{2}\left(K_{v}, \widehat{T}\right)^{D}
$$


qui a une image dense; l'application $\theta$ est continue via la remarque 3.5

Il suffit alors de montrer que l'image de l'application composée

$$
\prod_{v \in S} T\left(K_{v}\right) \rightarrow \prod_{v \in S} H^{2}\left(K_{v}, \widehat{T}\right)^{D} \rightarrow \amalg_{S}^{2}(\widehat{T})^{D}
$$

coïncide avec celle de l'application continue

$$
\prod_{v \in S} H^{2}\left(K_{v}, \widehat{T}\right)^{D} \rightarrow \amalg_{S}^{2}(\widehat{T})^{D} .
$$

D'après le lemme 9.6, l'image de l'application composée ci-dessus est finie. La densité de l'image de $\theta$ assure alors que l'image de l'application composée coïncide avec celle de $\prod_{v \in S} H^{2}\left(K_{v}, \widehat{T}\right)^{D} \rightarrow \amalg_{S}^{2}(\widehat{T})^{D}$.

Corollaire 9.9. - On a une suite exacte de groupes abéliens

$$
0 \rightarrow \overline{T(K)} \rightarrow \prod_{v \in \Omega_{K}} T\left(K_{v}\right) \rightarrow \amalg_{\omega}^{2}(\widehat{T})^{D} \rightarrow \amalg^{2}(\widehat{T})^{D} \rightarrow 0,
$$

où l'image de l'application $\prod_{v \in \Omega_{K}} T\left(K_{v}\right) \rightarrow \amalg_{\omega}^{2}(\widehat{T})^{D}$ est un groupe fini. Plus précisément, soit $S$ un ensemble fini de places contenant toutes les places de mauvaise réduction de $T$. Alors $A_{S}(T)=A(T)$ et $\amalg_{S}^{2}(\widehat{T})=\amalg_{\omega}^{2}(\widehat{T})$.

Démonstration. - Soit $S^{\prime}$ ensemble fini de places avec $S \subset S^{\prime}$. On a alors un diagramme commutatif

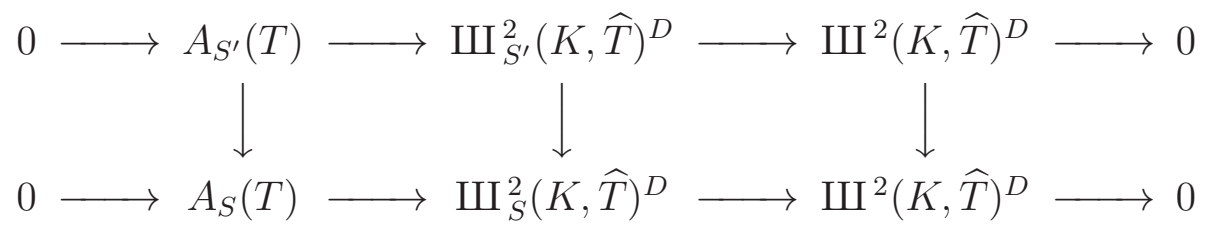

La flèche de droite est un isomorphisme, la flèche de gauche est une surjection de groupes finis. Dès que $S$ contient toutes les places de mauvaise réduction de $T$, la proposition 9.1 montre que la projection $A_{S^{\prime}}(T) \rightarrow A_{S}(T)$ est un isomorphisme. On conclut qu'il en est de même pour la flèche verticale médiane, et donc que pour $S$ contenant toutes les places de mauvaise réduction pour $T$, on a $\amalg_{S}^{2}(K, \widehat{T})=\amalg_{\omega}^{2}(K, \widehat{T})$.

Remarque 9.10. - Il se peut très bien que les deux groupes $\amalg_{\omega}^{2}(\widehat{T})$ et $\amalg^{2}(\widehat{T})$ soient infinis, mais $\amalg^{2}(\widehat{T})$ est toujours d'indice fini dans $\amalg_{\omega}^{2}(\widehat{T})$ par le corollaire 9.9. Les deux groupes sont égaux pour un tore quasi-trivial, et plus précisément égaux si et seulement si l'approximation faible vaut pour $T$. Noter aussi qu'on ne peut pas comme dans le cas où $K$ est un corps de 
nombres identifier le dual de $\amalg^{2}(\widehat{T})$ avec $\amalg^{1}(T)$ car en général $\amalg^{2}(\widehat{T})$ contient une partie divisible non triviale.

Le théorème suivant est le substitut, dans le présent contexte, de la suite exacte de Voskresenskiǐ pour les tores sur un corps de nombres ([32], Chap. 4, Section 11.6, Th. page 120, ou encore [28], Th. 9.2.ii) et [[8], Prop. 9.5).

Théorème 9.11. - Soit $K=\mathbb{C}((t))(C)$ comme ci-dessus, et soit $T$ un $K$ tore. Soit $A(T)$ le groupe fini mesurant le défaut d'approximation faible et $\amalg(T)$ le groupe fini mesurant le défaut du principe de Hasse pour les espaces principaux homogènes sous T. On a une suite exacte de groupes finis

$$
0 \rightarrow A(T) \rightarrow\left[\amalg_{\omega}^{2}(K, \widehat{T}) / \mathrm{Div}\right]^{D} \rightarrow \amalg^{1}(K, T) \rightarrow 0 .
$$

Démonstration. - La démonstration du corollaire ci-dessus établit que le groupe $\amalg^{2}(K, \widehat{T})$ est d'indice fini dans $\amalg_{\omega}^{2}(K, \widehat{T})$. Leurs sous-groupes divisibles maximaux coïncident donc. Comme $A(T)$ est fini, du corollaire 9.9 on déduit une suite exacte de groupes finis

$$
0 \rightarrow A(T) \rightarrow\left[\amalg_{\omega}^{2}(K, \widehat{T}) / \mathrm{Div}\right]^{D} \rightarrow\left[\amalg^{2}(K, \widehat{T}) / \mathrm{Div}\right]^{D} \rightarrow 0 .
$$

Le théorème 7.2 identifie le groupe $\left[\amalg^{2}(K, \widehat{T}) / \mathrm{Div}\right]^{D}$ avec $\amalg^{1}(K, T)$.

On a une inclusion $\amalg_{\text {cyc }}^{2}(\widehat{T}) \subset \amalg_{\omega}^{2}(\widehat{T})$, donc une application

$$
\amalg_{\text {сус }}^{2}(\widehat{T}) \rightarrow \amalg_{\omega}^{2}(\widehat{T}) / \operatorname{Div}
$$

qui induit une application

$$
\left[\amalg_{\omega}^{2}(\widehat{T}) / \mathrm{Div}\right]^{D} \rightarrow \amalg_{\text {сус }}^{2}(\widehat{T})^{D} .
$$

On a un isomorphisme entre $\amalg_{\text {cyc }}^{2}(\widehat{T})$ et le sous-groupe $B r_{e} T_{c}$ du groupe de Brauer non ramifié d'une compactification lisse $T_{c}$ de $T$ formé des éléments nuls en l'élément neutre. L'application composée

$$
A(T) \rightarrow \mathrm{Br}_{e} T_{c}^{D}
$$

est induite par l'accouplement de réciprocité (cf. [16], preuve du Th. 6.1.). On va voir qu'en général cette application n'est pas injective, en construisant maintenant un exemple qui montre que, contrairement au cas où $K$ est un corps de nombres, le défaut d'approximation faible d'un $K$-tore n'est pas toujours contrôlé par son groupe de Brauer non ramifié (cette assertion étant valide une fois la compatibilité ci-dessus vérifiée). 
Lemme 9.12. - Il existe une courbe sur $\mathbb{C}((t))$ de corps de fonctions $K$, telle que $K$ possède deux extensions galoisiennes $K_{1}, K_{2}$ de groupes de Galois respectifs $\mathbb{Z} / 4$ et $\mathbb{Z} / 2$, vérifiant : l'extension $K_{1} / K$ est totalement décomposée en tout point fermé de $C$ et l'extension $K_{2} / K$ a au moins un sous-groupe de décomposition non cyclique.

Démonstration. - Soit $E$ la courbe elliptique sur $\mathbb{C}$ d'équation affine $y^{2}=$ $x(x-1)(x-2)$. Soit $E^{\prime} \rightarrow E$ une isogénie de noyau $\mathbb{Z} / 4$; soit $X \rightarrow E$ un revêtement galoisien de groupe $(\mathbb{Z} / 2)^{2}$, totalement ramifié en un certain point $m \in E(\mathbb{C})$, par exemple le revêtement donné par $\sqrt{x-x_{0}}, \sqrt{y-y_{0}}$, où $y_{0}^{2}=x_{0}\left(x_{0}-1\right)\left(x_{0}-2\right) \neq 0$. Soient $K, K_{1}, K_{2}$ les corps de fonctions respectifs de de $E \times_{\mathbb{C}} \mathbb{C}((t)), E^{\prime} \times_{\mathbb{C}} \mathbb{C}((t)), X \times_{\mathbb{C}} \mathbb{C}((t))$. Alors $K_{1} / K$ est totalement décomposé en toute place de $v$ car le revêtement $E^{\prime} \rightarrow E$ est étale au-dessus du corps algébriquement clos $\mathbb{C}$, donc totalement décomposé partout. De plus, en la place $v$ de $E \times_{\mathbb{C}} \mathbb{C}((t))$ correspondant au point fermé audessus de $m$, l'extension $\left(K_{2}\right)_{v} / K_{v}$ est totalement ramifiée, donc son groupe de Galois reste $(\mathbb{Z} / 2)^{2}$.

Lemme 9.13. - Il existe un module galoisien fini $M$ sur $K$ tel que le groupe $\amalg_{\omega}^{1}(M)$ contienne strictement $\amalg^{1}(M)+\amalg_{\text {cyc }}^{1}(M)$.

Démonstration. - On commence par prendre (via le lemme précédent) des extensions $K_{1}, K_{2}$ de $K$, de groupes de Galois respectifs $D=\mathbb{Z} / 4$ et $H=$ $(\mathbb{Z} / 2)^{2}$, vérifiant :

a) $K_{1} / K$ est totalement décomposée en tout point fermé $v$ de $C$.

b) $K_{2} / K$ a au moins un sous-groupe de décomposition (disons en $v_{0}$ ) non cyclique, i.e. égal à $H$.

Les extensions galoisiennes $K_{1}$ et $K_{2}$ de $K$ sont linéairement disjointes vu que $K_{1}$ est totalement décomposée en $v_{0}$ tandis que $K_{2}$ est inerte.

Posons $L=K_{1} \cdot K_{2}$, c'est une extension galoisienne de $K$ de groupe $G=$ $D \times H \simeq \mathbb{Z} / 4 \times(\mathbb{Z} / 2)^{2}$, où $H=H \times 1$ est le groupe de Galois de $L$ sur $K_{1}$, et $D=1 \times D$ est le groupe de Galois de $L$ sur $K_{2}$.

Rappelons que presque tous les groupes de décomposition sont cycliques. L'hypothèse a) donne que tous les groupes de décomposition dans $G$ sont inclus dans $H$.

Soit $M$ un $G$-module fini. Soit $\alpha \in H^{1}(G, M)$. Alors :

i) Pour qu'on ait $\alpha \in \amalg^{1}(G, M)$, il faut que la restriction $\alpha_{H}$ à $H$ soit nulle car le groupe de décomposition $G_{v_{0}}$ est $H$. 
ii) Pour qu'on ait $\alpha \in \amalg_{\text {cyc }}^{1}(G, M)$, il faut que $\alpha_{D}$ soit nulle car $D$ est un sous-groupe cyclique de $G$.

iii) Pour qu'on ait $\alpha \in \amalg_{\omega}^{1}(G, M)$, il suffit que l'on ait $\alpha_{D^{\prime}}=0$ pour tout sous-groupe cyclique $D^{\prime}$ de $H$, puisque pour presque toute $v, G_{v}$ est l'un de ces sous-groupes.

En prenant alors pour $M$ le noyau de l'augmentation $\mathbb{Z} / 16[G] \rightarrow \mathbb{Z} / 16$, on obtient (cf. Sansuc [28], p. 22) :

$$
\amalg^{1}(G, M) \subset \mathbb{Z} / 4 ; \quad \amalg_{\text {сус }}^{1}(G, M) \subset \mathbb{Z} / 4 ; \quad \amalg_{\omega}^{1}(G, M)=\mathbb{Z} / 8 .
$$

On a de fait $H^{1}\left(G, I_{G}\right)=\mathbb{Z} / 16$, le premier et le second groupe sont dans $4 .(\mathbb{Z} / 16)$, et le troisième est 2.(Z/Z 16$)$. Ceci montre que $\amalg_{\omega}^{1}(G, M)$ contient strictement le groupe $\amalg_{\text {cyc }}^{1}(G, M)+\amalg^{1}(G, M)$. On observe alors qu'on a $\amalg_{\text {cyc }}^{1}(K, M) \subset \amalg_{\text {сус }}^{1}(G, M)$ car l'image de tout élément de $\amalg_{\text {cyc }}^{1}(K, M)$ dans $H^{1}(L, M)$ est dans $\amalg_{\text {cyc }}^{1}(L, M)$, qui est nul (rappelons que le module galoisien $M$ est déployé par $L$ ), donc appartient à $H^{1}(G, M) \cap \amalg_{\text {cyc }}^{1}(K, M)=\amalg_{\text {cyc }}^{1}(G, M)$. Soit alors $\alpha$ un élément de $\amalg_{\omega}^{1}(G, M)$ qui n'est pas dans $\amalg_{\text {cyc }}^{1}(G, M)+\amalg^{1}(G, M)$, alors $\alpha$ ne peut pas s'écrire $\beta+\gamma$ avec $\beta \in \amalg_{\text {cyc }}^{1}(K, M)$ et $\gamma \in \amalg^{1}(K, M)$, sinon $\gamma$ serait aussi dans $\amalg^{1}(G, M)$ d'après ce qui précède. Finalement $\amalg_{\omega}^{1}(K, M)$ contient strictement $\amalg_{\text {cyc }}^{1}(K, M)+\amalg^{1}(K, M)$.

Proposition 9.14. - Il existe un K-tore T tel que le complexe

$$
0 \rightarrow \overline{T(K)} \rightarrow \prod_{v \in \Omega_{K}} T\left(K_{v}\right) \rightarrow \amalg_{\mathrm{cyc}}^{2}(\widehat{T})^{D}
$$

ne soit pas exact.

Démonstration. - Pour tout module galoisien fini $M$, le groupe $\amalg_{\omega}^{1}(M)$ est fini (voir Prop. 4.6). Comme ce groupe est la réunion des $\amalg_{S}^{1}(M)$ pour $S$ fini, il existe un ensemble fini $S$ de places telle que $\amalg_{S}^{1}(K, M)=\amalg_{\omega}^{1}(M)$.

Soit $M$ le module galoisien fini du lemme précédent, et soit $S$ fini tel que $\amalg_{S}^{1}(M)=\amalg_{\omega}^{1}(M)$. Soit $F$ le dual de $M$. On plonge $F$ dans un tore quasitrivial $R$ et on note $T:=R / F$ le tore quotient. On a donc une suite exacte de modules galoisiens de type fini

$$
0 \rightarrow \widehat{T} \rightarrow \widehat{P} \rightarrow M \rightarrow 0 .
$$

Quitte à agrandir $S$, on peut d'après ce qui a été vu plus haut, supposer $\amalg_{S}^{2}(\widehat{T})=\amalg_{\omega}^{2}(\widehat{T})$. 
On a un diagramme commutatif

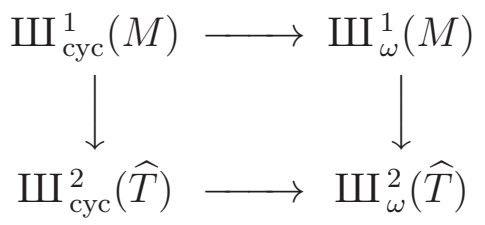

où les flèches horizontales sont des inclusions. Comme les groupes $H^{1}(K, \widehat{R})$, $H^{1}\left(K_{v}, \widehat{R}\right)$ et $\amalg_{\text {cyc }}^{2}(\widehat{R})$ sont nuls, les flèches verticales sont injectives, et $\amalg_{\text {cyc }}^{1}(M) \stackrel{\sim}{\rightarrow} \amalg_{\text {cyc }}^{2}(\widehat{T})$.

Comme le groupe fini $\amalg_{\omega}^{1}(M)$ contient strictement $\amalg^{1}(M)+\amalg_{\text {сус }}^{1}(M)$, il existe un élément non nul $f$ de $\amalg_{S}^{1}(M)^{D}$ qui est non nul, mais s'annule sur $\amalg^{1}(M)+\amalg_{\text {cyc }}^{1}(M)$.

La suite exacte

$$
H^{1}(K, F) \rightarrow \prod_{v \in S} H^{1}\left(K_{v}, F\right) \rightarrow \amalg_{S}^{1}(M)^{D} \rightarrow \amalg^{1}(M)^{D} \rightarrow 0
$$

(proposition 9.3) permet donc de relever $f$ en un $\left(f_{v}\right) \in \prod_{v \in S} H^{1}\left(K_{v}, F\right)$. Comme $H^{1}\left(K_{v}, R\right)=0$, ce $\left(f_{v}\right)$ se relève lui-même en un élément $\left(t_{v}\right)$ de $\prod_{v \in S} T\left(K_{v}\right)$. On a un diagramme commutatif de complexes

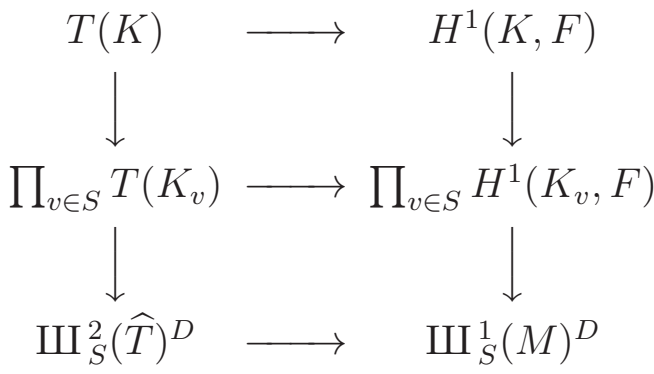

Maintenant, on observe que :

a) Le composé

$$
\prod_{v \in S} T\left(K_{v}\right) \rightarrow \amalg_{S}^{2}(\widehat{T})^{D}=\amalg_{\omega}^{2}(\widehat{T})^{D} \rightarrow \amalg_{\mathrm{cyc}}^{2}(\widehat{T})^{D}
$$

s'annule sur l'élément $\left(t_{v}\right)$, car $f$ s'annule sur $\amalg_{\text {cyc }}^{1}(M)$, et on a aussi un isomorphisme $\amalg_{\text {cyc }}^{1}(M) \stackrel{\simeq}{\rightarrow} \amalg_{\text {cyc }}^{2}(\widehat{T})$.

b) L'élément $\left(t_{v}\right)$ n'est pas dans l'adhérence de $T(K)$. Sinon son image $\left(f_{v}\right) \in \prod_{v \in S} H^{1}\left(K_{v}, F\right)$ proviendrait de $H^{1}(K, F)$, et d'après la proposition 9.3 l'image $f$ de $\left(f_{v}\right)$ dans $\amalg_{\omega}^{1}(M)^{D}$ serait nulle, ce qui n'est pas le cas par construction. 
Remarque 9.15. - A contrario, on voit facilement que si $T$ est un tore admettant une résolution comme ci-dessus mais avec $M=\widehat{F}$ vérifiant $\amalg_{\omega}^{1}(M)=\amalg^{1}(M)+\amalg_{\text {cyc }}^{1}(M)$, alors le complexe

$$
0 \rightarrow \overline{T(K)} \rightarrow \prod_{v \in \Omega_{K}} T\left(K_{v}\right) \rightarrow \amalg_{\mathrm{cyc}}^{2}(\widehat{T})^{D}
$$

est bien exact.

Corollaire 9.16. - Il existe un K-tore flasque $Q$ tel que le complexe

$$
H^{1}(K, Q) \rightarrow \bigoplus_{v \in \Omega} H^{1}\left(K_{v}, Q\right) \rightarrow H^{1}(K, \widehat{Q})^{D}
$$

ne soit pas exact.

On n'a donc pas de suite exacte de Poitou-Tate "complète" pour un tore dans notre cadre.

Démonstration. - Soient $K$ et $T$ comme dans la proposition 9.14 Considérons une résolution flasque

$$
1 \rightarrow Q \rightarrow R \rightarrow T \rightarrow 1 \rightarrow 0
$$

de $T$. Notons déjà que le tore $Q$ étant flasque, on a, pour $S$ ensemble fini de places contenant toutes les places de mauvaise réduction

$$
H^{1}(K, \widehat{Q})=\amalg_{\text {сус }}^{1}(\widehat{Q})=\amalg_{\omega}^{1}(\widehat{Q})=\amalg_{S}^{1}(\widehat{Q})
$$

car un tore flasque qui est déployé par une extension cyclique est facteur direct d'un tore quasi-trivial. Par ailleurs on a un diagramme commutatif à lignes exactes

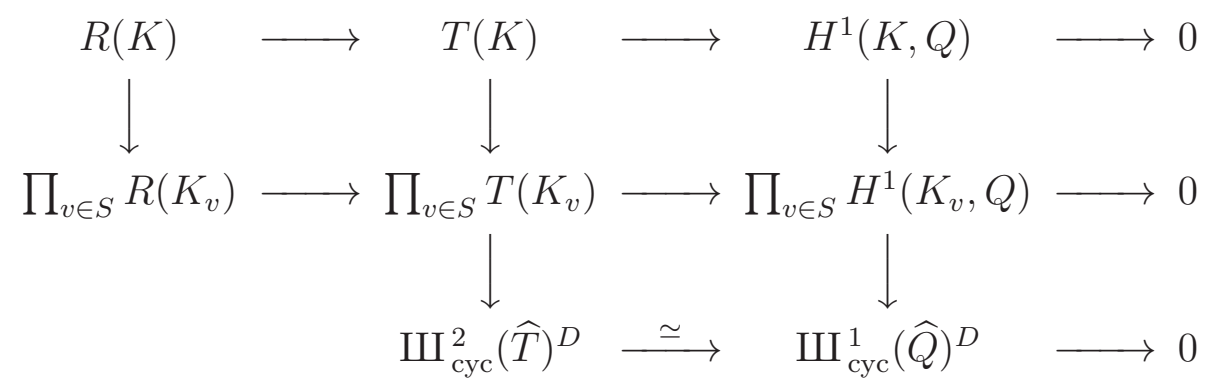

et la proposition 9.14 nous dit qu' on peut prendre $S$ tel que la suite

$$
\overline{T(K)}_{S} \rightarrow \prod_{v \in S} T\left(K_{v}\right) \rightarrow \amalg_{\mathrm{cyc}}^{2}(\widehat{T})^{D}
$$


ne soit pas exacte. Comme $R(K)$ est dense dans $\prod_{v \in S} R\left(K_{v}\right)$, une chasse au diagramme donne alors que la dernière colonne ne peut pas être exacte,

Remarque 9.17. - Comme nous l'a signalé T. Szamuely, la théorie du corps de classes fonctionne bien sur un corps du type $k(C)$, avec $k$ quasi fini, à condition d'avoir la condition supplémentaire $H^{1}(k, J)=0$, où $J$ est la jacobienne de la courbe $C$ (voir [26] et [24], Chapitre I, Appendice A), condition qui est vérifiée si $k$ est fini mais pas toujours si $k=\mathbb{C}((t))$. La proposition 9.3 permet de faire le lien avec cette théorie : en effet cette propostion combinée au théorème 6.2 pour $F=\mu_{n}$ donne une suite exacte :

$$
K^{*} / K^{*^{n}} \rightarrow \prod_{v} K_{v}^{*} / K_{v}^{*^{n}} \rightarrow \Gamma_{K}^{\mathrm{ab}} / n \rightarrow \amalg^{2}\left(\mu_{n}\right) \rightarrow 0
$$

où $\Gamma_{K}$ est le groupe de Galois absolu de $K$ et $\prod^{\prime}$ désigne le produit restreint. Soit $I_{K}=\prod^{\prime} K_{v}^{*}$ le groupe des idèles de $K$ et $C_{K}=I_{K} / K^{*}$ son groupe des classes d'idèles. La nullité de $H^{1}(k, J)$ implique celle de $\amalg^{2}\left(\mu_{n}\right)$ (propositions 2.1 iii) et 2.9 ii)), ce qui permet sous cette hypothèse d'en déduire facilement qu'on a une application de réciprocité $C_{K} \rightarrow \Gamma_{K}^{\mathrm{ab}}$ d'image dense, comme dans le cas classique où $k$ est fini. Sans la nullité de $\amalg^{2}\left(\mathbb{G}_{m}\right)$, on voit qu'il subsiste une obstruction à la densité de l'application de réciprocité (obstruction qui disparaît si on remplace $K$ par son extension abélienne maximale totalement décomposée en tous les points fermés de $C$ ).

\section{Groupes algébriques linéaires}

Soit $K=\mathbb{C}((t))(C)$ le corps des fonctions d'une courbe $C$ projective, lisse, géométriquement connexe sur le corps $\mathbb{C}((t))$. On note $K_{v}$ le complété de $K$ en une place $v \in \Omega$, où $\Omega$ est l'ensemble des places associées aux points fermés de $C$.

10.1. Rappels. - Dans [4], on a décrit de nombreuses propriétés des groupes algébriques linéaires connexes sur divers corps de "dimension" 2, mais le cas des corps $\mathbb{C}((t))(C)$ n'y avait pas été pris en considération. Ceci fut rectifié dans l'exposé de Parimala [25]. Les énoncés qui suivent sont pour l'essentiel contenus dans le $\S 6$ et le $\S 7$ de son exposé. Leur démonstration utilise les propriétés des corps $\mathbb{C}((t))(C)$ rappelés au $\$ 1$ du présent article : propriété $C_{2}$, et coïncidence de l'exposant et de l'indice des algèbres simples centrales sur un tel corps.

Soit $G$ un $K$-groupe algébrique linéaire connexe. 
- Les quotients $G(K) / R$ et $G\left(K_{v}\right) / R$ par la $R$-équivalence sont finis. Si $G$ est simplement connexe, ils sont triviaux.

- Pour $G$ semisimple simplement connexe sur un corps $K=\mathbb{C}((t))(C)$ et $S$ un ensemble fini de points fermés $v$ de $C$, on a l'approximation faible : l'application naturelle

$$
G(K) \rightarrow \prod_{v \in S} G\left(K_{v}\right)
$$

a une image dense. Pour le voir, il suffit d'adapter la démonstration de [4, Thm. 4.7].

- Si $G$ est simplement connexe, on a $H^{1}(K, G)=0$ et $H^{1}\left(K_{v}, G\right)=0$. C'est un cas particulier de [4, Thm. 1.2].

- Un $K$-groupe semisimple simplement connexe sans facteur de type $A_{n}$ est une $K$-variété $K$-rationnelle. C'est un cas particulier de [4, Thm. 1.5; Thm. 4.3].

10.2. Obstruction au principe local-global pour les espaces principaux homogènes. - Le théorème suivant (qui s'étend immédiatement au cas non réductif par trivialité de la cohomologie galoisienne des unipotents en caractéristique zéro) est l'analogue du théorème 8.5 de Sansuc [28].

On utilise ici les notations introduites aux paragraphes 8.1 et 8.2 .

Théorème 10.1. - Si G est un K-groupe linéaire connexe réductif, l'ensemble $\amalg^{1}(K, G)$ est fini.

On a un accouplement

$$
\amalg^{1}(K, G) \times \mathrm{Б}(G) \rightarrow \mathbb{Q} / \mathbb{Z},
$$

fonctoriel et multiplicatif en $G$, compatible à toute application $\amalg^{1}(K, G) \rightarrow$ $\amalg^{2}(K, \mu)$ déduite d'une $K$-isogénie $H \rightarrow G$ de noyau $\mu$.

Cet accouplement est non dégénéré à gauche. Si G est semisimple, l'accouplement est non dégénéré des deux côtés.

Pour E un espace principal homogène sous $G$, ayant des points dans tous les $K_{v}$, on a un isomorphisme naturel $\theta: \mathrm{B}(E) \rightarrow \mathrm{B}(G)$ et pour $\alpha \in \mathrm{B}(E)$, l'obstruction au principe de Hasse sur $E$ associée à $\alpha$, soit $\rho_{E}(\alpha) \in \mathbb{Q} / \mathbb{Z}$, coïncide avec $<E, \theta \alpha>\in \mathbb{Q} / \mathbb{Z}$.

Démonstration. - (esquisse) La méthode étant analogue à celle de [28], nous allons nous contenter d'en rappeler les grandes étapes. On trouvera une autre méthode, basée sur l'hypercohomologie des complexes de tores, dans [22] ; cette autre méthode donne en outre le fait que le noyau à droite de l'accouplement est le sous-groupe divisible maximal de $\mathrm{Б}(G)$. 
Une suite exacte

$$
1 \rightarrow \mu \rightarrow H \times{ }_{K} P \rightarrow G \rightarrow 1,
$$

avec $H$ semisimple simplement connexe, $P$ un $K$-tore quasitrivial et $\mu$ un $K$-groupe fini central dans $H \times P$ définit ce que l'on appelle un revêtement spécial d'un $K$-groupe réductif $G$. Pour tout $K$-groupe réductif $G$, il existe un entier $n>0$ tel que $G^{n}$ admette un tel revêtement ([28], Lemme 1.10). Compte tenu des annulations $H^{1}(K, P)=0$ ( $P$ quasitrivial) et $H^{1}(K, H)=$ 0 (vu ci-dessus), une telle suite exacte induit une application

$$
\amalg^{1}(K, G) \rightarrow \amalg^{2}(K, \mu)
$$

dont le noyau est trivial.

D'après Sansuc on a un isomorphisme ([28, Cor. 7.4])

$$
H^{1}(K, \widehat{\mu}) \stackrel{\sim}{\rightarrow} \operatorname{Ker}\left[\operatorname{Br}_{a}(G) \rightarrow \operatorname{Br}_{a}\left(H \times_{K} P\right)\right]
$$

et un isomorphisme ([28, Lemme 6.9])

$$
\left.\operatorname{Br}_{a}\left(H \times_{K} P\right)\right] \stackrel{\simeq}{\rightarrow} H^{2}(K, \widehat{P}),
$$

ceci sur tout corps $K$ et de façon fonctorielle en le corps $K$. On obtient ainsi une suite exacte

$$
0 \rightarrow \amalg^{1}(K, \widehat{\mu}) \rightarrow \mathrm{Б}(G) \rightarrow \amalg^{2}(K, \widehat{P}) .
$$

Le théorème 6.2 fournit une dualité de groupes finis

$$
\amalg^{1}(K, \widehat{\mu}) \times \amalg^{2}(K, \mu) \rightarrow \mathbb{Q} / \mathbb{Z} .
$$

On définit alors comme dans [28], Prop. 8.1) un accouplement

$$
\amalg^{1}(K, G) \times \mathrm{Б}(G) \rightarrow \mathbb{Q} / \mathbb{Z}
$$

à la Brauer-Manin, en utilisant le fait que $B(E)=B(G)$ pour $E$ un espace principal homogène de $G([\mathbf{2 8}]$, Lemme 6.8). L'accouplement ainsi défini est compatible avec celui ci-dessus via $\amalg^{1}(K, \widehat{\mu}) \rightarrow \mathrm{D}(G)$ (preuve identique à celle de [28], Th 8.5).

On obtient en fin de compte que l'accouplement

$$
\amalg^{1}(K, G) \times \mathrm{Б}(G) \rightarrow \mathbb{Q} / \mathbb{Z}
$$

est non dégénéré à gauche. Pour $E$ un espace principal homogène sous un $K$ groupe réductif, l'obstruction de Brauer-Manin associée à $\operatorname{Br}(E)$ (et même à son sous-groupe $B(E)$ ) est la seule obstruction au principe de Hasse.

Si enfin $G$ est supposé semi-simple, alors son revêtement simplement connexe

$$
1 \rightarrow \mu \rightarrow \widetilde{G} \rightarrow G \rightarrow 1
$$


induit, compte tenu des propriétés des corps $K$ et $K_{v}$ mentionnées en 10.1 , une bijection $\amalg^{1}(K, G) \stackrel{\widetilde{\Im}}{\rightarrow} \amalg^{2}(K, \mu)$ (cas particulier de [11], Th. 5.7.i). D'après Sansuc $\left([\mathbf{2 8}]\right.$, Th. 7.2) et compte tenu du fait que $\operatorname{Br}_{a}(\widetilde{G})=0$ sur tout corps $K$ (car $\widetilde{G}$ est semi-simple simplement connexe), on a alors un isomorphisme $\amalg^{1}(K, \hat{\mu}) \stackrel{\widetilde{ }}{\rightarrow} \mathrm{D}(G)$, ce qui implique que l'accouplement

$$
\amalg^{1}(K, G) \times \mathrm{B}(G) \rightarrow \mathbb{Q} / \mathbb{Z}
$$

est dans ce cas non dégénéré des deux côtés.

\section{Références}

[1] S. Bosch, M. Raynaud et W. Lütkebohmert, Néron models, Ergebnisse der Mathematik und ihrer Grenzgebiete, 3. Folge, 21, Springer-Verlag, Berlin, 1990.

[2] J.-L. Colliot-Thélène, Birational invariants, purity and the Gersten conjecture, in $K$-Theory and Algebraic Geometry: Connections with Quadratic Forms and Division Algebras, AMS Summer Research Institute, Santa Barbara 1992, ed. W. Jacob and A. Rosenberg, Proceedings of Symposia in Pure Mathematics 58, Part I (1995) $1-64$.

[3] J.-L. Colliot-Thélène, Groupe de Brauer non ramifié d'espaces homogènes de tores, Journal de théorie des nombres de Bordeaux, à paraître.

[4] J.-L. Colliot-Thélène, P. Gille et R. Parimala, Arithmetic of linear algebraic groups over 2-dimensional geometric fields, Duke Math. J. 121 (2004) 285-341.

[5] J.-L. Colliot-Thélène, R. Parimala et V. Suresh, Patching and local-global principles for homogeneous spaces over function fields of $p$-adic curves, Commentarii Math. Helv. 87 (2012) no. 4, 1011-1033.

[6] J.-L. Colliot-Thélène, R. Parimala et V. Suresh, Lois de réciprocité supérieures et points rationnels, prépublication, novembre 2013.

[7] J.-L. Colliot-Thélène et J.-J. Sansuc, Cohomologie des groupes de type multiplicatif sur les schémas réguliers, C. R. Acad. Sc. Paris 287 (1978) 449-452.

[8] J.-L. Colliot-Thélène et J.-J. Sansuc, Homogeneous spaces under flasque tori : applications, J. Algebra 106 (1987) 148-205.

[9] H. Esnault et O. Wittenberg, On the cycle class map for zero-cycles over local fields, avec un appendice par S. Bloch, prépublication, février 2014.

[10] P. Gille et A. Pianzola, Isotriviality and étale cohomology of Laurent polynomial rings, Journal of Pure and Applied Algebra 212 (2008) 780-800.

[11] C. Gonzaléz-Avilés : Quasi-abelian crossed modules and nonabelian cohomology, prépublication 2013. 
[12] A. Grothendieck, M. Artin, J.-L. Verdier : Séminaire de géométrie algébrique, Théorie des topos et cohomologie étale des schémas (SGA 4), III, Lecture notes in Math. 305, Springer-Verlag 1973

[13] A. Grothendieck, Le groupe de Brauer I, II, III, in Dix exposés sur la cohomologie des schémas, Masson \& North-Holland, Paris, 1968.

[14] D. Harari, C. Scheiderer et T. Szamuely, Weak approximation for tori over $p$ adic function fields, à paraître dans Internat. Math. Res. Notices.

[15] D. Harari et T. Szamuely, Arithmetic duality theorems for 1-motives, J. reine angew. Math. 578 (2005), 93-128. Corrigenda, J. reine angew. Math. 632 (2009), 233-236.

[16] D. Harari et T. Szamuely, Local-global principles for 1-motives, Duke Math. J. 143, no 3 (2008), 531-557.

[17] D. Harari et T. Szamuely, Local-global questions for tori over $p$-adic function fields, à paraître dans J. Algebraic Geom.

[18] D. Harbater, J. Hartmann et D. Krashen, Application of patching to quadratic forms and central simple algebras, Inv. math. 178 2009) 231-263.

[19] D. Harbater, J. Hartmann, D. Krashen, Local-global principles for torsors over arithmetic curves, prépublication 2011.

[20] D. Harbater, J. Hartmann, D. Krashen, Local-global principles for Galois cohomology, à paraître dans Comment. Math. Helv.

[21] G. Harder, Halbeinfache Gruppenschemata über Dedekindringen, Invent. math. 4 (1967), 165-191.

[22] D. Izquierdo, Théorèmes de dualité arithmétique et approximation faible pour les corps de fonctions sur des corps locaux supérieurs, prépublication 2014.

[23] J. S. Milne, Étale Cohomology, Princeton University Press, 1980.

[24] J. S. Milne, Arithmetic duality theorems, Second Edition, Kea Books (2006).

[25] R. Parimala, Arithmetic of linear algebraic groups over two-dimensional fields, Exposé au congrès international, Hyderabad, India, 2010.

[26] D. S. Rim, G. Whaples, Global norm-residue map over quasi-finite field, Nagoya Math. J. 27 (1966), 323-329.

[27] D. Saltman, Division algebras over $p$-adic curves, J. Ramanujan Math. Soc. 12 (1997) 25-47. Correction to Division algebras over p-adic curves, J. Ramanujan Math. Soc. 13 (1998), no. 2, 125-129.

[28] J-J. Sansuc, Groupe de Brauer et arithmétique des groupes algébriques linéaires sur un corps de nombres, J. reine angew. Math. 327 (1981), 12-80.

[29] J-P. Serre, Cohomologie Galoisienne, Cinquième édition, révisée et complétée, Springer L.N.M. 5 (1994). 
[30] A. N. Skorobogatov, Torsors and rational points, Cambridge Tracts in Mathematics 144, Cambridge University Press, Cambridge, 2001.

[31] V. E. Voskresenskiǐ, Propriétés birationnelles des groupes algébriques linéaires (en russe), Izv. Akad. Nauk SSSR Ser. Mat. 34 (1970) 3-19.

[32] V. E. Voskresenskiň, Algebraic groups and their birational invariants, Translations of Mathematical Monographs 179, American Math. Soc. (1998).

16 avril 2014

Jean-Louis Colliot-ThéLÈne, C.N.R.S., Université Paris Sud, Mathématiques,

Bâtiment 425, 91405 Orsay Cedex, France • E-mail: jlct@math.u-psud.fr

DAVID HARARI, Université Paris Sud, Mathématiques, Bâtiment 425, 91405 Orsay Cedex,

France - E-mail:David.Harari@math.u-psud.fr 